Seleção de modelos cópula-GARCH: uma abordagem bayesiana

\author{
João Luiz Rossi
}


Data de Depósito:

Assinatura:

\title{
Seleção de modelos cópula-GARCH: uma abordagem bayesiana
}

\author{
João Luiz Rossi
}

Orientador: Prof. Dr. Ricardo Sandes Ehlers

Dissertação apresentada ao Instituto de Ciências Matemáticas e de Computação - ICMC-USP, como parte dos requisitos para obtenção do título de Mestre em Ciências - Ciências de Computação e Matemática Computacional. VERSÃO REVISADA

USP - São Carlos

Junho de 2012 
Ficha catalográfica elaborada pela Biblioteca Prof. Achille Bassi e Seção Técnica de Informática, ICMC/USP, com os dados fornecidos pelo(a) autor(a)

\begin{tabular}{|c|c|}
\hline \multirow[t]{3}{*}{ R831s } & $\begin{array}{l}\text { Rossi, João Luiz } \\
\quad \text { Seleção de modelos cópula-GARCH: uma abordagem } \\
\text { bayesiana / João Luiz Rossi; orientador Ricardo } \\
\text { Sandes Ehlers. -- São Carlos, } 2012 \text {. } \\
\quad 79 \text { p. }\end{array}$ \\
\hline & $\begin{array}{l}\text { Dissertação (Mestrado - Programa de Pós-Graduação en } \\
\text { Ciências de Computação e Matemática Computacional) -- } \\
\text { Instituto de Ciências Matemáticas e de Computação, } \\
\text { Universidade de São Paulo, } 2012 \text {. }\end{array}$ \\
\hline & $\begin{array}{l}\text { 1. Cópulas. 2. Seleção de modelos. 3. GARCH. } 4 . \\
\text { Modelos assimétricos. 5. DIC. I. Ehlers, Ricardo } \\
\text { Sandes, orient. II. Título. }\end{array}$ \\
\hline
\end{tabular}


Dedico este trabalho à Andrea Correa Varella 


\section{Agradecimentos}

Agradeço à Deus por me dar saúde e sabedoria.

À minha mãe Aparecida e a minhas irmãs Luana e Luceli por sempre me incentivarem.

Aos pais da minha namorada, Euclydes Varella Filho e Maria Helena Correa Varella, por me apoiarem em todos os sentidos.

Ao meu orientador Prof. Ricardo Sandes Ehlers pelos ensinamentos e conselhos.

Ao professor Marinho Gomes de Andrade por todos os auxílios prestados.

Aos membros da banca examinadora professor Luiz Koodi Hotta e professor Marinho Gomes de Andrade pelos comentários, sugestões e contribuições.

Aos professores da estatística do ICMC-USP pelos ensinamentos e por toda atenção que me deram.

Aos funcionários do ICMC-USP.

À CAPES pelo apoio financeiro.

Ao professor Antonio Sergio Ferraudo pela ajuda na preparação para pós-graduação. Aos alunos José Augusto Fioruci, Willian Luís de Oliveira, José Julio Flores Delgado e aos demais alunos da pós-graduação em estatística por toda ajuda que me deram.

Ao LCCA-Laboratório de Computação Científica Avançada da Universidade de São Paulo pelo auxílio computacional prestado na pesquisa desenvolvida. 


\section{Resumo}

Esta dissertação teve como objetivo o estudo de modelos para séries temporais bivariadas, que tem a estrutura de dependência determinada por meio de funções de cópulas. A vantagem desta abordagem é que as cópulas fornecem uma descrição completa da estrutura de dependência. Em termos de inferência, foi adotada uma abordagem Bayesiana com utilização dos métodos de Monte Carlo via cadeias de Markov (MCMC). Primeiramente, um estudo de simulações foi realizado para verificar como os seguintes fatores, tamanho das séries e variações nas funções de cópula, nas distribuições marginais, nos valores do parâmetro de cópula e nos métodos de estimação, influenciam a taxa de seleção de modelos segundo os critérios EAIC, EBIC e DIC. Posteriormente, foram realizadas aplicações a dados reais dos modelos com estrutura de dependência estática e variante no tempo.

Palavras-chave: Cópulas, seleção de modelos, DIC, GARCH, modelos assimétri$\cos$. 


\begin{abstract}
The aim of this work was to study models for bivariate time series, where the dependence structure among the series is modeled by copulas. The advantage of this approach is that copulas provide a complete description of dependence structure. In terms of inference was adopted the Bayesian approach with utilization of Markov chain Monte Carlo (MCMC) methods. First, a simulation study was performed to verify how the factors, length of the series and variations on copula functions, on marginal distributions, on copula parameter value and on estimation methods, may affect models selection rate given by EAIC, EBIC and DIC criteria. After that, we applied the models with static and time-varying dependence structure to real data.
\end{abstract}

Key words: Copulas, model selection, DIC, GARCH, asymmetric models. 


\section{Sumário}

1 Introdução 1

1.1 Motivação . . . . . . . . . . . . . . . . . . . . . 1

1.2 Conceitos básicos . . . . . . . . . . . . . . . . . 3

1.2.1 Função de Cópula . . . . . . . . . . . . . . 3

1.2.2 Medidas de Dependência . . . . . . . . . . . . . 5

1.3 Revisão bibliográfica . . . . . . . . . . . . . . 7

2 Modelos com Dependência Estática e Estimação dos Parâmetros 11

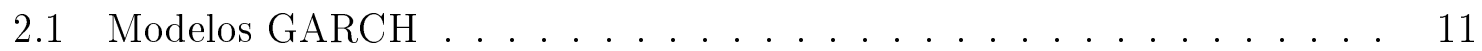

2.2 Modelos de Cópula . . . . . . . . . . . . . . . . . 16

2.3 Modelos Cópula-GARCH . . . . . . . . . . . . . . 20

2.4 Estimação e Inferência . . . . . . . . . . . . . . . . . . 22

2.4.1 O Algoritmo de Metropolis-Hastings . . . . . . . . . . . 22

2.4.2 Estimação em Um e Dois Passos . . . . . . . . . . . . . . . 25

2.5 Geração de séries aleatórias . . . . . . . . . . . 26 
4.1 Especificação dos modelos . . . . . . . . . . . . . . 36

4.2 Critérios para seleção de modelos . . . . . . . . . . . . . 37

4.3 Resultados e Conclusões . . . . . . . . . . . . . . . . . . . 38

5 Aplicações $\quad 53$

5.1 Especificação dos modelos . . . . . . . . . . . . . . 53

5.2 O conjunto de dados . . . . . . . . . . . . . . . . 54

5.3 Resultados . . . . . . . . . . . . . . . . 55

5.4 Conclusões e Considerações Finais . . . . . . . . . . . . . 67

$\begin{array}{ll}\text { Referências Bibliográficas } & 68\end{array}$ 


\section{Capítulo 1}

\section{Introdução}

\subsection{Motivação}

Atualmente, existe muito interesse em tentar modelar a dependência temporal de retornos financeiros utilizando-se de modelos de cópula. A principal característica desta abordagem é que a distribuição conjunta dos retornos é quebrada em dois componentes, as funções de densidade marginais e a estrutura de dependência, (ver por exemplo Ausin \& Lopes, 2010; Arakelian \& Dellaportas, 2012). Além disso, a quantidade de modelos univariados disponível é muito grande quando comparada a quantidade de modelos multivariados. Por meio do uso de cada função cópula é possível criar um modelo multivariado, a partir de quaisquer distribuições marginais. Deste modo, ao se trabalhar com cópulas, o numero de possíveis distribuições conjuntas cresce consideravelmente e distribuições multivariadas flexíveis e não elípticas podem ser obtidas.

Outro fato a ser observado é a ampla utilização do coeficiente de correlação de Pearson como medida de dependência entre variáveis aleatórias. Há evidência empírica reportada na literatura recente de que o uso de cópulas permite uma descrição mais 
realista do comportamento de dependência entre as séries, que vai além deste coeficiente de correlação (Embrechts \& Straumann, 2002; Embrechts \& McNeil, 2003).

A título de ilustração, foram geradas observações de variáveis aleatórias bidimensionais, cujas distribuições marginais são normais padrão e o coeficiente de correlação é igual a 0.5 (Figura 1.1). Nota-se que cada uma das distribuições conjuntas apresenta uma estrutura de dependência diferente entre as marginais e esta pode ser capturada por meio do uso das cópulas.
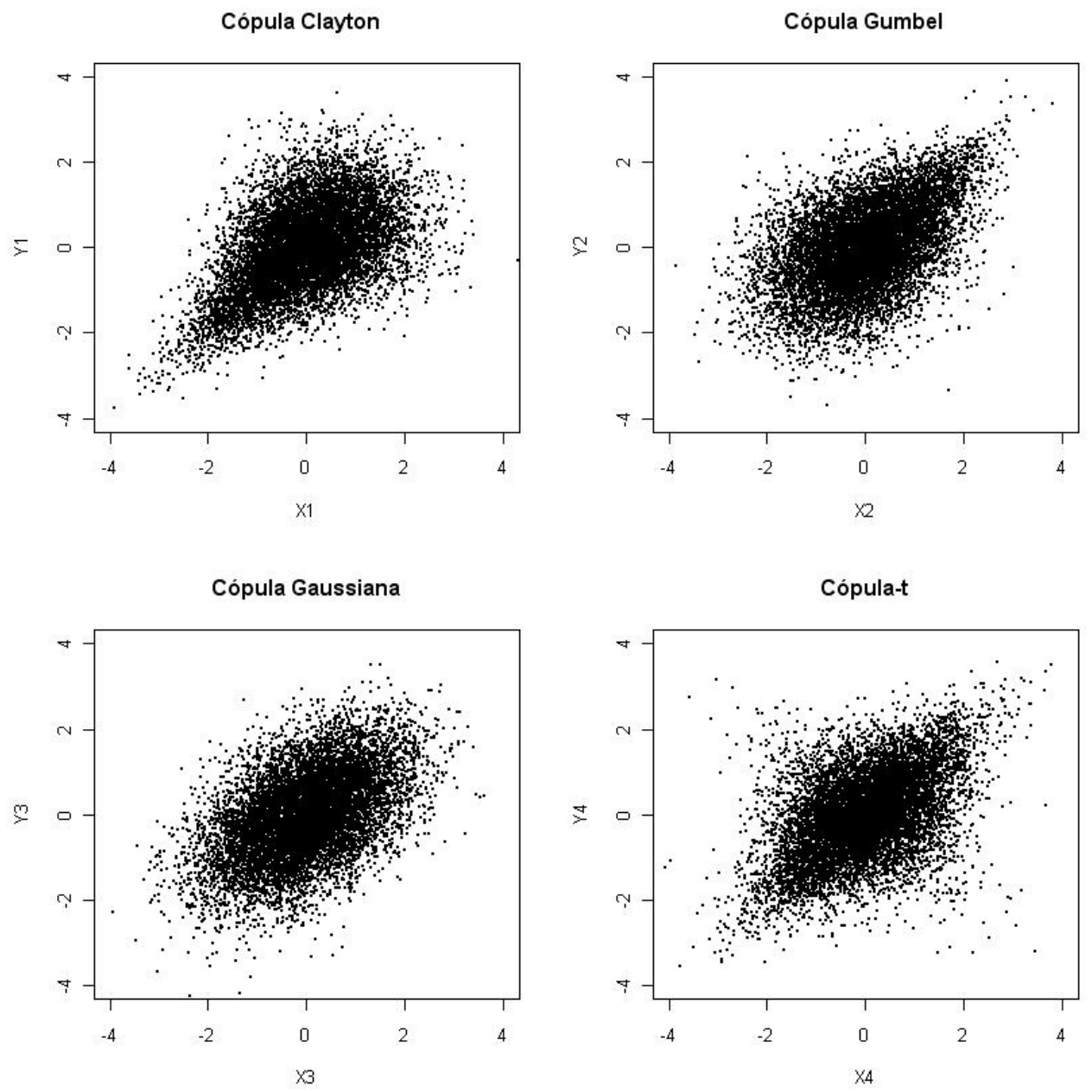

Figura 1.1: 10000 observações de distribuições conjuntas obtidas de marginais normais padrão, por meio da utilização das cópulas Clayton, Gumbel, Normal e T. Para os quatro casos o coeficiente de correlação da distribuição conjunta é igual a 0.5 , porém são apresentadas diferentes estruturas de dependência. 
Então, neste trabalho, o nosso objetivo foi estudar modelos bivariados, obtidos por meio da utilização de cópulas e distribuições marginais GARCH, os modelos cópulaGARCH. Além disso, objetivou-se estudar suas extensões e formas de comparação entre estes modelos, utilizando uma abordagem Bayesiana.

A principal contribuição deste trabalho são os estudos de simulação, realizados no contexto dos modelos cópula-GARCH (capítulo 4). Estes estudos foram realizados para verificar a taxa de seleção dos modelos, em diferentes circunstancias, segundo critérios estatísticos conhecidos. Além dos estudos, são apresentadas no capítulo 5, aplicações à dados reais. Para estas aplicações foram utilizados os modelos com dependência estática e dependência variante no tempo.

\subsection{Conceitos básicos}

Uma breve exposição dos conceitos básicos, necessários à compreensão dos tópicos relacionados à dissertação, é apresentada nesta seção. Estes conceitos podem ser encontrados em Nelsen (2006), que apresenta uma introdução à teoria das cópulas.

\subsubsection{Função de Cópula}

Definição 1.1. Sejam $S_{1}, S_{2} \ldots, S_{n}$ subconjuntos não vazios do $\overline{\mathbb{R}}^{n}$, onde $\overline{\mathbb{R}}$ é o conjunto dos reais estendido $[-\infty, \infty]$, seja $C$ uma função n-dimensional tal que $\operatorname{DomC}=S_{1} \times S_{2} \times \ldots \times S_{n}$. Seja $B=[\mathbf{a}, \mathbf{b}]=\left[a_{1}, b_{1}\right] \times \ldots \times\left[a_{n}, b_{n}\right]$ com $a_{i}<b_{i}$ para todo $i$, um retângulo $n$-dimensional com vértices $\boldsymbol{w}=w_{1}, w_{2}, \ldots, w_{2^{n}}$ em DomC, então o C-volume de $B$ é dado por

$$
V_{C}=\sum \operatorname{sgn}(\boldsymbol{w}) C(\boldsymbol{w})
$$


na qual a soma é calculada para todos os vértices $\boldsymbol{w}$ de $B$, e sgn(w) é dado por

$$
\operatorname{sgn}(\boldsymbol{w})=\left\{\begin{array}{c}
1, \quad \text { se } w_{k}=a_{k} \text { para um numero par de } k^{\prime} s \\
-1, \quad \text { se } w_{k}=a_{k} \text { para um numero impar de } k^{\prime} s .
\end{array}\right.
$$

Definição 1.2. Seja C uma função com dominio em $[0,1]^{n}$ e imagem em $[0,1]$, dizemos que C é uma cópula n-dimensional se:

1. Para todo $\mathbf{u} \in[0,1]^{n}, C(\mathbf{u})=0$, se pelo menos uma das coordenadas de $\mathbf{u}$ é 0 , e $C(\mathbf{u})=u_{k}$ se todas as coordenadas de $\mathbf{u}$ exceto a k-ésima são iguais a 1 .

2. Para todo $\mathbf{u}, \mathbf{v} \in[\mathbf{0}, \mathbf{1}]^{\mathbf{n}}$ tais que $u_{i} \leq v_{i}$ para todo $i, V_{C}([\mathbf{u}, \mathbf{v}]) \geq \mathbf{0}$ onde $V_{C} e^{\prime}$ o C-volume.

Apesar da grande quantidade de trabalhos recentes sobre o tema, o teorema que segue foi demonstrado para o caso bivariado em 1959 e é base da teoria de cópulas.

Teorema 1.1. (Teorema de Sklar) Sejam $X_{1}, X_{2}, \ldots, X_{n}$ variáveis aleatórias com funções de distribuição acumulada $F_{1}, F_{2}, \ldots, F_{n}$ respectivamente e distribuição acumulada conjunta $H$. Então existe uma função $C$ tal que,

$$
H\left(x_{1}, x_{2}, \ldots, x_{n}\right)=C\left(F_{1}\left(x_{1}\right), F_{2}\left(x_{2}\right), \ldots, F_{n}\left(x_{n}\right)\right)
$$

Para todo $\left(x_{1}, x_{2}, \ldots, x_{n}\right) \in \overline{\mathbb{R}}^{n}$, Se $F_{1}, F_{2}, \ldots, F_{n}$ são continuas, $C$ é única, caso contrario $C$ é determinada somente em $I m F_{1} \times \ldots \times \operatorname{Im} F_{n}$. Reciprocamente se $C$ é uma n-cópula e $F_{1}, F_{2}, \ldots, F_{n}$ são distribuições acumuladas, então a função $H$ é uma distribuição conjunta n-dimensional com marginais $F_{1}, F_{2}, \ldots, F_{n}$.

Corolário 1.1. Seja H uma função de distribuição acumulada conjunta com marginais $F_{1}, F_{2}, \ldots, F_{n}$ continuas e função de cópula $C$. Então, para todo $\mathbf{u}=\left(u_{1}, u_{2}, \ldots, u_{n}\right) \in$ $[0,1]^{n}$ 


$$
C\left(u_{1}, u_{2}, \ldots, u_{n}\right)=H\left(F_{1}^{-1}\left(u_{1}\right), F_{2}^{-1}\left(u_{2}\right), \ldots, F_{n}^{-1}\left(u_{n}\right)\right)
$$

A partir da função de cópula $C$ e das acumuladas marginais $F_{1}, F_{2}, \ldots F_{n}$, se $H$ é uma função continua, a função de densidade conjunta $h$, com funções de densidade marginais $f_{1}, f_{2}, \ldots, f_{n}$, pode ser obtida por meio da utilização da relação abaixo, proveniente do Teorema 1.1,

$$
h\left(x_{1}, x_{2}, \ldots, x_{n}\right)=c\left(F_{1}\left(x_{1}\right), F_{2}\left(x_{2}\right), \ldots, F_{n}\left(x_{n}\right)\right) \prod_{i=1}^{n} f_{i}\left(x_{i}\right)
$$

na qual $c$ é a função densidade de cópula.

Se a densidade conjunta $h$ e as marginais são conhecidas, a densidade de cópula $c$ pode ser obtida por meio da expressão abaixo que deriva do Corolário 1.1

$$
c\left(u_{1}, u_{2}, \ldots, u_{n}\right)=\frac{h\left(F_{1}^{-1}\left(u_{1}\right), F_{2}^{-1}\left(u_{2}\right), \ldots, F_{n}^{-1}\left(u_{n}\right)\right)}{\prod_{i=1}^{n} f_{i}\left(F_{i}^{-1}\left(u_{i}\right)\right)} .
$$

\subsubsection{Medidas de Dependência}

Pode-se afirmar, que o coeficiente de correlação de Pearson, dado por,

$$
\rho_{X Y}=\frac{E(X Y)-E(X) E(Y)}{\sqrt{\operatorname{Var}(X) \operatorname{Var}(Y)}} .
$$

é a medida mais utilizada para se quantificar o grau de associação entre variáveis aleatórias. Porém, algumas limitações são evidenciadas ao se utilizar o coeficiente de correlação como medida de dependência (Embrechts \& Straumann, 2002). Como, por exemplo, o fato de $\rho_{X Y}=0$ não implicar em independência, que ocorre quando a dependência é não linear, ou o fato de $\rho_{X Y}$ não ser invariante a transformações não 
lineares $\left(\rho_{X Y} \neq \rho_{T(X) T(Y)}\right.$ para $\mathrm{T}($.$) uma transformação não linear).$

As cópulas elípticas, Normal e T-student, tem como parâmetro que quantifica o grau de dependência entre as distribuições marginais o coeficiente de correlação de Pearson. Por outro lado, foram utilizadas distribuições conjuntas obtidas por meio de cópulas arquimedianas e estas foram reparametrizadas em função de medidas de dependência nas caudas, para aplicações do capítulo 5. As medidas de dependência nas caudas são definidas da seguinte maneira (Joe, 1997):

Definição 1.3. Sejam $X_{1}$ e $X_{2}$ variáveis aleatórias com funções de distribuição acumulada $F$ e G, respectivamente, e função de distribuição acumulada conjunta $C\left(F\left(X_{1}\right), G\left(X_{2}\right)\right)$. Se o limite $\lim _{\varepsilon \rightarrow 0^{+}} P\left[Y \leq G^{-1}(\varepsilon) \mid X \leq F^{-1}(\varepsilon)\right]=\lambda_{L}$ existe e $\left.\left.\in\right] 0,1\right]$, dizemos que a cópula $C$ possui dependência na cauda inferior igual a $\lambda_{L}$. De forma análoga se o limite $\lim _{\delta \rightarrow 1^{-}} P\left[Y>G^{-1}(\delta) \mid X>F^{-1}(\delta)\right]=\lambda_{U}$ existe $\left.\left.e \in\right] 0,1\right]$, dizemos que a cópula $C$ possui dependência na cauda superior igual a $\lambda_{U}$.

Um fato interessante é a possibilidade de se determinar essas medidas utilizando-se das funções de cópula,

$$
\lambda_{L}=\lim _{\varepsilon \rightarrow 0} \frac{C(\varepsilon, \varepsilon)}{\varepsilon}, \quad \quad \lambda_{U}=\lim _{\delta \rightarrow 1} \frac{1-2 \delta+C(\delta, \delta)}{1-\delta} .
$$

Outras medidas de dependência podem ser encontradas na literatura, como por exemplo, o Tau de Kendall $(\tau)$ e o Rho de Spearman $\left(\rho_{s}\right)$. Neste trabalho utilizouse apenas o Tau de Kendall, que pode ser interpretado como a diferença entre as probabilidades de concordância e a de discordância de duas variáveis aleatórias. Esta medida também pode ser determinada por meio da função de cópula,

$$
\begin{aligned}
\tau & =P\left(\left(X_{1}-X_{2}\right)\left(Y_{1}-Y_{2}\right)>0\right)-P\left(\left(X_{1}-X_{2}\right)\left(Y_{1}-Y_{2}\right)<0\right) \\
& =4 \int_{0}^{1} \int_{0}^{1} C\left(u_{1}, u_{2}\right) c\left(u_{1}, u_{2}\right) d u_{1} d u_{2}-1,
\end{aligned}
$$


com $\left(X_{1}, Y_{1}\right)$ e $\left(X_{2}, Y_{2}\right)$ pares independentes, identicamente distribuídos de variáveis aleatórias e $u_{1}, u_{2} \in[0,1]$.

\subsection{Revisão bibliográfica}

Existe um grande número de trabalhos recentes, nos quais a estrutura de dependência entre variáveis é especificada por modelos de cópula. Os estudos de simulação realizados no presente trabalho são semelhantes aos realizados por Silva \& Lopes (2008). Esses autores empregaram uma metodologia bayesiana com a finalidade de realizar estudos de simulação. Distribuições multivariadas são produzidas por meio da utilização das cópulas Clayton, Frank, Normal, Gumbel, Heavy tail, t de Student e das distribuições marginais exponencial, normal e normal assimétrica.

Dentre os estudos de simulação realizados pelos autores, daremos destaque a dois. No primeiro, são geradas amostras da distribuição conjunta, com marginais iguais e cada uma das funções de cópula. Então, são verificadas as taxas de acerto na seleção do modelo de cópula correto, segundo o critério DIC (Deviance Information Criterion), para amostras de tamanho 100, 200 e 500. No segundo estudo, os autores propõem comparar as taxas de seleção do modelo de cópula correto, quando os parâmetros são estimados separadamente (primeiramente os parâmetros das distribuições marginais e posteriormente o da função de cópula), com as taxas obtidas por meio da estimação em conjunto.

Os autores puderam concluir que, paralelamente aos critérios de informação comumente utilizados (EAIC e EBIC), pode-se obter bons resultados por meio da utilização do DIC na seleção do modelo de cópula apropriado. Além disso, sob uma perspectiva bayesiana, os autores recomendam que os parâmetros sejam estimados em conjunto, para que seja levada em consideração a dependência entre eles. Os resultados 
do artigo são limitados ao uso das cópulas uniparamétricas e distribuições marginais empregadas no estudo.

Outros artigos discutem as diferentes maneiras de fazer com que a estrutura de dependência varie ao longo do tempo. Rodriguez (2007), propõe estudar o contágio financeiro entre mercados por meio do uso de modelos de cópula, que tem seus parâmetros variando em uma cadeia de Markov com o número de estados pré-fixado. Para as distribuições marginais, os autores utilizaram os modelos SWARCH(2,1) (Switching ARCH), que é uma adaptação do modelo ARCH (Autoregressive Conditional Heteroskedastic). Estes modelos permitiram identificar os dois regimes de volatilidade pré-definidos, o de baixa volatilidade e alta volatilidade.

Para as estruturas de dependência, os autores utilizaram a cópula Student-t e misturas das cópulas Clayton, Gumbel e Frank, determinadas por meio da atribuição de pesos a cada uma delas. Os pesos para Clayton, Gumbel, Frank e os parâmetros, graus de liberdade e coeficiente de correlação para t-student, também podiam variar de acordo com os diferentes estados da cadeia. Foram analisados índices diários de retornos de cinco países do leste da Ásia, durante crises asiáticas, e de quatro países da América Latina durante a crise do México. Testes de razão de verossimilhança foram utilizados para verificar se havia mudança significativa nos parâmetros para os diferentes regimes de volatilidade.

Em Arakelian \& Dellaportas (2012) a associação entre mercados é especificada em diferentes regimes de contágio, por meio de um modelo fronteira (threshold), que permite que a função e o parâmetro de cópula mudem ao longo do tempo. Para esta abordagem, os autores consideraram o número de regimes desconhecido ao invés de pré-estipulado e as densidades marginais foram avaliadas por meio do método Monte Carlo via cadeias de Markov, com aproximação de Laplace. Ao modelar as distribuições marginais, estes autores assumiram que a evolução da volatilidade condicional seguia 
um processo GARCH(1,1). As cópulas utilizadas foram a Frank, a Clayton e a Gumbel. Os resultados obtidos mostraram que o método MCMC com saltos reversíveis (Green, 1995) pode ser utilizado para determinar, de forma efetiva, o número de regimes de contágio e, com a utilização de funções de cópula, especificar a estrutura de dependência ao longo do tempo.

Patton (2006a) testa a assimetria em um modelo de dependência. Para isto, o autor propõe uma extensão da teoria de cópulas que permite o uso variáveis condicionais, com o objetivo de construir modelos flexíveis para a dependência condicional entre retornos. Nas cópulas Normal e Joe-Clayton Simetrizada (uma pequena modificação da Joe-Clayton original "BB7", que faz com que esta seja simétrica nos casos em que as dependências nas caudas são iguais), são introduzidas equações para que, fixada a amostra, os parâmetros de dependência evoluam de forma determinística ao longo do tempo. Este método de evolução dos parâmetros de cópula, segundo o autor, segue o espírito das funções de "densidade condicionais auto-regressivas", propostas por Hansen (1994). Para as distribuições marginais o autor utilizou modelos AR-GARCH com resíduos t de Student. Os modelos foram aplicados para estudar as associações entre as taxas diárias de câmbio do marco alemão e do iene japonês para o dólar americano.

Em Ausin \& Lopes (2010) os parâmetros do modelo de cópula variam ao longo do tempo seguindo uma equação de evolução, que depende dos seus valores anteriores e de observações das distribuições marginais ( $\operatorname{GARCH}(1,1)$ com erros t de Student). Já a distribuição conjunta foi obtida por meio da utilização da cópula-t variante no tempo. A abordagem adotada pelos autores para se fazer inferência sobre os parâmetros, predições e determinar medidas de risco, como Valor-em-risco (VaR) e Valor-em-risco Condicional (CVaR), é completamente bayesiana. A principal diferença deste para outros artigos é que neste, todos os parâmetros foram estimados em conjunto, ao invés de se utilizar o procedimento de estimação usual (parâmetros das marginais e do modelo de cópula estimados separadamente). Uma comparação entre os dois métodos 
de estimação é realizada pelos autores por meio da aplicação do modelo à um conjunto de dados gerado. Nesta aplicação os autores constataram uma redução no desvio padrão dos parâmetros, quando estes eram estimados em um passo. Posteriormente, a estimação em um passo é aplicada a um conjunto de dados reais (log-retornos dos índices diários Dow Jones e DAX) para se obter as estimativas das medidas de risco. Na conclusão, os autores propõem a realização de novos trabalhos com modelos alternativos para as distribuições marginais e funções de cópula.

Uma revisão detalhada das aplicações de teoria das cópulas à dados do mercado financeiro, pode ser encontrada em Patton (2008). No próximo capítulo são apresentados os modelos utilizados, a metodologia empregada na inferência sobre seus parâmetros e um algoritmo de geração para séries aleatórias, provenientes de modelos cópula-GARCH. 


\section{Capítulo 2}

\section{Modelos com Dependência Estática e}

\section{Estimação dos Parâmetros}

Neste capítulo, são apresentados os modelos com estrutura de dependência estática e a metodologia relacionada à estimação e inferência sobre seus parâmetros. A metodologia relacionada aos modelos com estrutura de dependência variante no tempo será apresentada no capítulo 3.

\subsection{Modelos GARCH}

Proposto por Bollerslev (1986), o modelo GARCH (Generalized Autoregressive Conditional Heteroskedastic) é uma generalização dos modelos ARCH (Engle, 1982), que acrescenta um termo que faz com que a volatilidade dependa também dos seus valores defasados. Apesar deste acréscimo, a generalização proporciona um grande ganho no sentido da parcimônia, pois, na maioria dos casos, um modelo GARCH possibilita melhor ajuste com menor quantidade de parâmetros, quando comparado ao modelo ARCH. 
$\mathrm{O}$ modelo $\operatorname{GARCH}(p, q)$ é definido da seguinte maneira,

$$
\begin{aligned}
& X_{t}=\sqrt{h_{t}} \varepsilon_{t} \\
& h_{t}=\omega+\sum_{i=1}^{p} \alpha_{i} X_{t-i}^{2}+\sum_{j=1}^{q} \beta_{j} h_{t-j},
\end{aligned}
$$

na qual, $\varepsilon_{t}$ é um processo puramente aleatório com média igual a zero e variância unitária, normalmente denominado "erros" do modelo. Aos parâmetros são impostas as seguintes condições de positividade e estacionariedade $\omega>0, \alpha_{i} \geq 0$ para $i=1,2, \ldots, p$, $\beta_{j} \geq 0$ para $j=1,2, \ldots, q$ e $\sum_{i=1}^{p} \alpha_{i}+\sum_{j=1}^{q} \beta_{j}<1$.

Seja $\boldsymbol{x}=\left(x_{1}, x_{2}, \ldots, x_{T}\right)$ uma série observada que segue um processo GARCH(p,q). Dado o vetor de parâmetros $\boldsymbol{\theta}=\left(\omega, \alpha_{1}, \ldots, \alpha_{p}, \beta_{1}, \ldots, \beta_{q}\right)$ e ao separar a densidade conjunta dos $k$ primeiros termos, $k=\max (p, q)$, a função de densidade conjunta pode ser escrita como,

$$
p(\boldsymbol{x} \mid \boldsymbol{\theta})=p\left(x_{1}, x_{2}, \ldots, x_{k} \mid \boldsymbol{\theta}\right) \prod_{t=k+1}^{T} p\left(x_{t} \mid x_{t-1}, \ldots, x_{t-k}, \boldsymbol{\theta}\right) .
$$

Geralmente o número de observações da série é grande o suficiente para que se desconsidere o termo $p\left(x_{1}, x_{2}, \ldots, x_{k} \mid \boldsymbol{\theta}\right)$. Deste modo, supondo que os erros tenham distribuição ST (standard Student-t, que é obtida por meio de uma pequena alteração na distribuição t de Student para que esta tenha variância unitária), ou seja,

$$
\varepsilon_{1}, \ldots, \varepsilon_{T} \stackrel{\mathrm{iid}}{\sim} S T_{\nu}
$$

para $T$ grande temos que,

$$
p(\boldsymbol{x} \mid \boldsymbol{\theta}) \cong \prod_{t=k+1}^{T} p\left(x_{t} \mid x_{t-1}, \ldots, x_{t-k}, \boldsymbol{\theta}\right)=\prod_{t=k+1}^{T} \frac{\Gamma\left(\frac{\nu+1}{2}\right)}{\sqrt{(\nu-2) \pi h_{t}} \Gamma\left(\frac{\nu}{2}\right)}\left(1+\frac{x_{t}^{2}}{(\nu-2) h_{t}}\right)^{-\frac{\nu+1}{2}}
$$

Na maioria dos casos o modelo GARCH de ordem $(1,1)$ é suficiente para conseguir 
um bom ajuste à dados do mercado financeiro, logo, este modelo foi utilizado no presente trabalho. Ao utilizar o $\operatorname{GARCH}(1,1)$ e aplicar a função log é possível obter a log-verossimilhança,

$$
\begin{aligned}
\ell\left(\boldsymbol{\theta} \mid \boldsymbol{x}_{\boldsymbol{t}}\right) & =\sum_{t=2}^{T} \log \left[\frac{\Gamma\left(\frac{\nu+1}{2}\right)}{\sqrt{(\nu-2) \pi h_{t}} \Gamma\left(\frac{\nu}{2}\right)}\left(1+\frac{x_{t}^{2}}{(\nu-2) h_{t}}\right)^{-\frac{\nu+1}{2}}\right] \\
& =(T-1)\left[\log \Gamma\left(\frac{\nu+1}{2}\right)-\log \Gamma\left(\frac{\nu}{2}\right)-\frac{1}{2}(\log (v-2)+\log \pi)\right] \\
& +\sum_{t=2}^{T} \log \frac{1}{h_{t}}-\frac{\nu+1}{2} \sum_{t=2}^{T} \log \left(1+\frac{x_{t}^{2}}{(\nu-2) h_{t}}\right) .
\end{aligned}
$$

Uma das aplicações mais comuns dos modelos GARCH é a modelagem de retorno de ativos. Os retornos podem ser definidos como $\ln \left(\frac{P_{t}}{P_{t-1}}\right)$, nos quais $P_{t}$ é o preço de um determinado ativo no tempo $t$. A título de exemplo, são apresentadas séries de valores $\left(P_{t}\right)$ e dos retornos $\left(r_{t}\right)$ de índices do mercado financeiro IBOVESPA e SP500 para o período de 03/01/2005 a 29/12/2011 (Figura 2.1). 

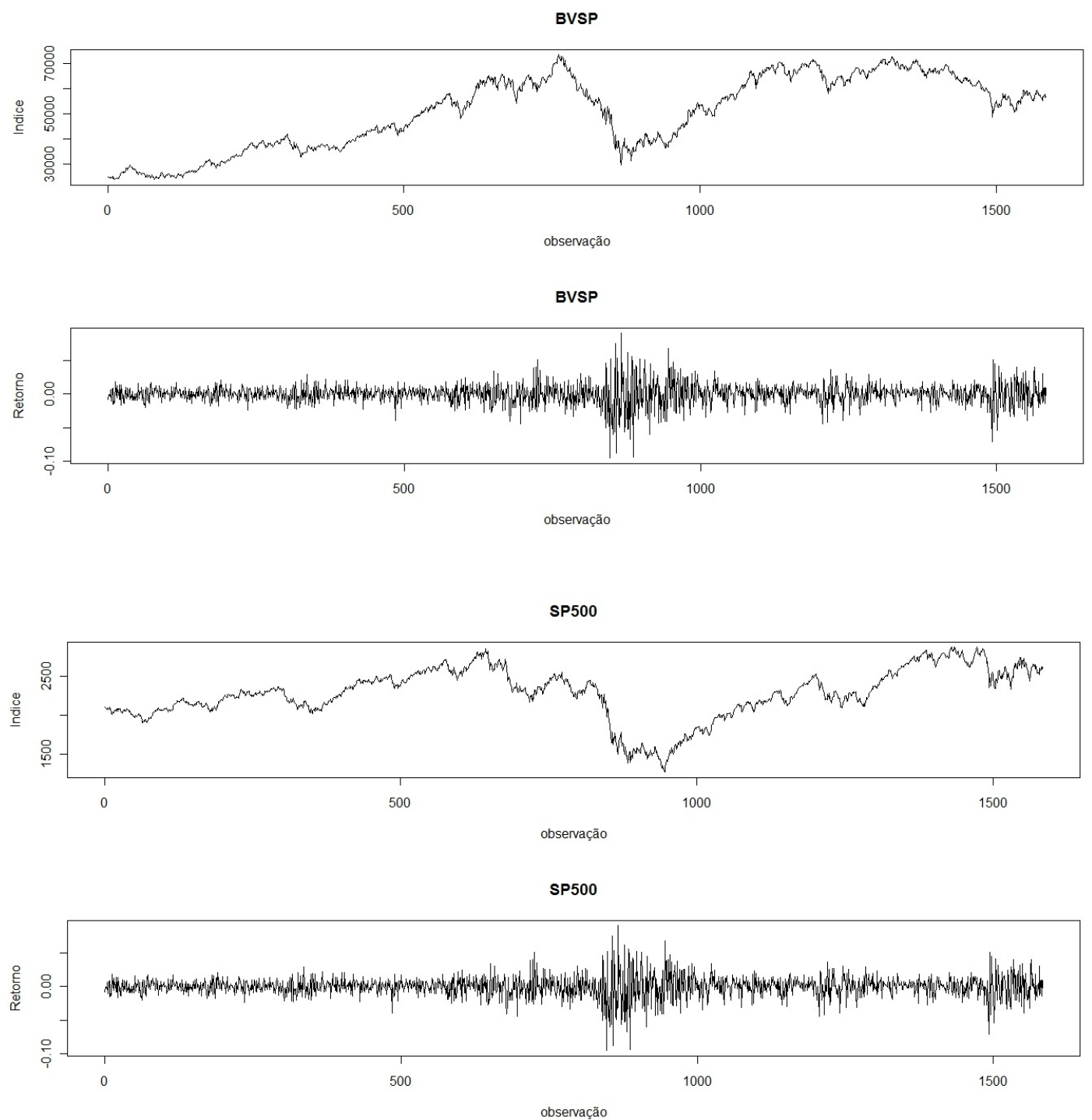

Figura 2.1: Séries dos valores $\left(P_{t}\right)$ e séries dos retornos $\left(r_{t}\right)$ dos índices IBOVESP (BVSP) do Brasil e Standard \& Poor's 500 (SP500) dos E.U.A. para o período de $03 / 01 / 2005$ a $29 / 12 / 2011$

Nas aplicações do GARCH à retornos de ativos é muito comum a uso da distribuição ST para erros do modelo. Esse fato deve-se às características que estes dados apresentam (como a curtose relativamente elevada), porém, o modelo com estes erros trata de forma simétrica os retornos. Deste modo, é possível que ao ajusta-lo aos dados, a hipótese de que os resíduos do modelo são ST seja rejeitada. Isso normalmente 
ocorre por um fato estilizado destas séries, o de que o volatilidade reage de forma diferente para aumentos e decréscimos nos valores defasados do ativo, conhecido como "efeito alavancagem". De modo geral, tem-se menor volatilidade para retornos defasados positivos e maior volatilidade para retornos defasados negativos.

São várias as propostas presentes na literatura para contornar esta limitação. A utilização dos modelos EGARCH (Nelson, 1991), por exemplo, seria uma alternativa. Neste trabalho, adotou-se a estratégia de atribuir distribuições assimétricas aos erros. Essa atribuição de assimetria pode ser realizada pela utilização das metodologias propostas por Azzalini (1985) e Fernandez \& Steel (1998), por exemplo. Neste trabalho, utilizou-se a metodologia apresentada por Fernandez \& Steel (1998), que possibilita a introdução de assimetria em determinados modelos pela equação,

$$
p(y \mid \gamma)=\frac{2}{\gamma+\frac{1}{\gamma}}\left\{f\left(\frac{y}{\gamma}\right) I_{[0, \infty)}(y)+f(y \gamma) I_{(-\infty, 0)}(y)\right\}
$$

na qual $\gamma$ é o parâmetro de assimetria, $f(\cdot)$ é a função de densidade. de um modelo qualquer, unimodal e simétrico em torno de $0, I_{[a, b]}$ é a função indicadora no intervalo $[a, b]$ e $p(y \mid \gamma)$ é a função de densidade. do modelo assimétrico obtido.

Ao aplicarmos a equação (2.3) à função de densidade de probabilidade da distribuição t de Student, obtemos uma distribuição assimétrica. Porém esta possui média e variância dadas pelas expressões,

$$
\mu_{\gamma}=m_{1}\left(\gamma-\frac{1}{\gamma}\right) \quad e \quad \sigma_{\gamma}^{2}=\left(1-m_{1}^{2}\right)\left(\gamma^{2}+\frac{1}{\gamma^{2}}\right)+2 m_{1}^{2}-1
$$

nas quais $m_{1}$ é o primeiro momento absoluto da distribuição t de Student dado por,

$$
m_{1}=\frac{2 \sqrt{\nu-2}}{\nu-1} \frac{\Gamma(1 / 2) \Gamma(\nu / 2)}{\Gamma((\nu+1) / 2)}
$$


Mas, para que uma distribuição seja atribuída aos erros, ela deve ter média zero e variância unitária. Deste modo, utilizou-se a versão padronizada da assimétrica obtida, que será denotada SST (standardized skewed Student- $t$ ) e tem função de densidade dada por,

$$
p(x)=\frac{2 \sigma_{\gamma}}{\gamma+1 / \gamma}\left\{f\left(\frac{\sigma_{\gamma} x+\mu_{\gamma}}{\gamma}\right) I_{\left(x \geq \frac{-\mu_{\gamma}}{\sigma \gamma}\right)}+f\left(\gamma\left(\sigma_{\gamma} x+\mu_{\gamma}\right)\right) I_{\left(x<\frac{-\mu_{\gamma}}{\sigma \gamma}\right)}\right\},
$$

na qual $f(\cdot)$ é a densidade da distribuição t de Student. A partir da densidade, a logverossimilhança do modelo GARCH é obtida de maneira análoga à apresentada para

a distribuição ST. Maiores detalhes sobre a obtenção da função de densidade SST podem ser encontrados em Ehlers (2012). Note que o modelo ST é obtido como caso particular quando $\gamma=1$.

A literatura sobre estudos de séries temporais é vasta e há uma grande quantidade de modelos disponíveis. Conceitos básicos, por exemplo, podem ser encontrados em Morettin \& Toloi (2004). Prosseguiremos, na próxima seção, com os conceitos relacionados à cópulas, objetivando a modelagem de séries de retornos bivariadas.

\subsection{Modelos de Cópula}

Vários modelos de cópula são apresentados em Nelsen (2006) e são muitas as variações propostas para estes modelos na literatura (exemplos podem ser encontrados em Patton, 2006b; Rodriguez, 2007). Para este trabalho, foram utilizados os modelos Normal e t de Student, da família de cópulas elípticas, e os modelos Clayton e Gumbel, da família de cópulas arquimedianas. Além destes, utilizou-se o modelo de cópula Heavy Tail, que é uma reparametrização da cópula Clayton de sobrevivência. Estes modelos foram escolhidos por serem amplamente utilizados na literatura e pelas diferentes características que cada um apresenta, pois possibilitam a modelagem dos 
vários aspectos da estrutura de dependência entre retornos.

As cópulas da família elíptica possuem dependência simétrica nas caudas $\left(\lambda_{L}=\lambda_{U}\right)$ e podem ser obtidas a partir da aplicação da equação (1.2), supondo um modelo elíptico para distribuições marginais e o mesmo modelo na sua versão multivariada para a distribuição conjunta (Método da inversão).

Cópula Normal $\left(C_{N}\right)$ - O parâmetro que quantifica o grau de dependência entre as distribuições marginais na $C_{N}$ é o coeficiente de correlação $(\rho)$, logo este pertence ao intervalo $[-1,1]$. A função de cópula Normal é dada pela expressão,

$$
C_{N}(u, v \mid \rho)=\int_{-\infty}^{\Phi^{-1}(u)} \int_{-\infty}^{\Phi^{-1}(v)} \frac{1}{2 \pi \sqrt{1-\rho^{2}}} \exp \left\{\frac{-\left(r^{2}-2 \rho r s+s^{2}\right)}{2\left(1-\rho^{2}\right)}\right\} d r d s .
$$

Aplicações relacionadas à cópula Normal podem ser encontradas em vários trabalhos, como por exemplo, em Patton (2006a) e Jondeau \& Rockinger (2006).

Cópula t de Student $\left(C_{T}\right)$ - É a função de cópula obtida pela aplicação da equação (1.2) à distribuições t de Student. A distribuição conjunta obtida por meio da utilização da $C_{T}$ na equação(1.1), será uma t bivariada, com graus de liberdade $\nu$, somente nos casos onde ambas as distribuições marginais forem t e essas, além da função de cópula, tiverem graus de liberdade também iguais a $\nu$. Sua função de distribuição é dada por,

$$
C_{T}(u, v \mid \rho, \nu)=\int_{-\infty}^{t_{\nu}^{-1}(u)} \int_{-\infty}^{t_{\nu}^{-1}(v)} \frac{1}{2 \pi \sqrt{1-\rho^{2}}}\left\{\frac{r^{2}-2 \rho r s+s^{2}}{\nu\left(1-\rho^{2}\right)}\right\}^{-\frac{\nu+2}{2}} d r d s .
$$

Os parâmetros da cópula T-student são o coeficiente de correlação $(\rho)$ e os graus de liberdade $(\nu>0)$. Neste trabalho o parâmetro $\nu$ foi fixado em quatro, assim como em Silva \& Lopes (2008), para que a cópula T tenha, necessariamente, aspectos diferentes da cópula $C_{N}$. Aplicações e conceitos relacionadas a cópula $C_{T}$ podem ser encontradas em Ausin \& Lopes (2010) e Demarta \& Mcneil (2004). 
As funções de cópula arquimedianas são obtidas a partir de uma função geradora $\varphi(\cdot)$ especifica para cada cópula. Esta função deve ser duas vezes diferenciável, com domínio no intervalo $[0,1]$ e imagem no intervalo $[0, \infty)$, que satisfaça as condições,

$$
\text { I) } \left.\varphi(0)=\infty, \quad \boldsymbol{I I}) \varphi(1)=0, \quad \boldsymbol{I I I}) \varphi^{\prime}(\cdot)<0 \text { e } \boldsymbol{I} \boldsymbol{V}\right) \varphi^{\prime \prime}(\cdot)>0
$$

Satisfeitas as condições impostas sobre a função $\varphi$, uma cópula da familia arquimediana é obtida por meio da seguinte expressão,

$$
C(u, v)=\varphi^{-1}(\varphi(u)+\varphi(v))
$$

Maiores informações sobre funções geradoras de cópulas arquimedianas e sobre as formas de se obter uma função de cópula podem ser encontradas em Nelsen (2006). São apresentadas abaixo, as funções de distribuição de cópulas arquimedianas que foram utilizadas $\left(C_{C}\right.$ e $\left.C_{G}\right)$.

Cópula Clayton $\left(C_{C}\right)$ - Tem dependência somente de cauda inferior. Sua função de distribuição é dada pela expressão,

$$
C_{C}\left(u, v \mid \delta_{C}\right)=\left(u^{-\theta_{C}}+v^{-\theta_{C}}-1\right)^{-\frac{1}{\theta_{C}}}
$$

com $\theta_{C}$ pertencente ao intervalo $[0, \infty)$. Informações sobre a cópula $C_{C}$ e suas propriedades podem ser encontradas em Clayton (1978).

Cópula Gumbel $\left(C_{G}\right)$ - Tem dependência somente de cauda superior. Sua função de distribuição é dada por,

$$
C_{G}\left(u, v \mid \theta_{G}\right)=\exp \left\{-\left((-\log u)^{\theta_{G}}+(-\log v)^{\theta_{G}}\right)^{\frac{1}{\theta_{G}}}\right\}
$$


com $\theta_{-} G$ pertencente ao intervalo $[1, \infty)$.

Cópula Heavy Tail $\left(C_{H}\right)$ - A cópula $C_{H}$ tem dependência somente de calda superior. Conforme mencionado anteriormente, ela é obtida por meio de uma reparametrização da cópula Clayton de sobrevivência, ou seja, para $\theta_{H}=1 / \theta_{C}$, a cópula Heavy Tail é dada por,

$$
C_{H}\left(u, v \mid \theta_{H}\right)=u+v-1+C_{C}\left(1-u, 1-v \mid \theta_{H}\right)
$$

ou seja,

$$
C_{H}\left(u, v \mid \theta_{H}\right)=u+v-1+\left((u-1)^{-1 / \theta_{H}}+(v-1)^{-1 / \theta_{H}}-1\right)^{-\theta_{H}},
$$

com $\theta_{H}$, parâmetro que quantifica o grau de dependência entre as distribuições marginais, pertencente ao intervalo $[0, \infty)$. Outro fato que diferencia a $C_{H}$ das quatro cópulas mencionadas anteriormente, é que nela a dependência entre as distribuições marginais aumenta conforme $\theta_{H}$ diminui.

A tabela 2.1 exibe algumas funções que relacionam as medidas de dependência, utilizadas na dissertação, aos parâmetros das cópulas utilizadas.

Uma forma direta de aplicação da teoria das cópulas, que faz uso de séries temporais, é a construção de modelos bivariados cujas marginais seguem um modelo do tipo GARCH. Este assunto será tratado de forma detalhada na próxima seção. 
Tabela 2.1: Medidas de dependência para as funções de cópula, tau de kendall $(\tau)$, dependência de cauda inferior $\left(\lambda_{L}\right)$ e dependência de cauda superior $\left(\lambda_{U}\right)$. T $\nu+1$ é a função de distribuição acumulada da t de Student com $\nu+1$ graus de liberdade.

\begin{tabular}{cccc}
\hline Cópula & $\tau$ & $\lambda_{\mathbf{L}}$ & $\lambda_{\mathbf{U}}$ \\
\hline $\mathbf{C}_{\mathbf{N}}$ & $\frac{2}{\pi} \arcsin \left(\rho_{N}\right)$ & - & - \\
$\mathbf{C}_{\mathbf{T}}$ & $\frac{2}{\pi} \arcsin \left(\rho_{T}\right)$ & $2 T_{\nu+1}\left(-\sqrt{\frac{(\nu+1)\left(1-\rho_{T}\right)}{1+\rho_{T}}}\right)$ & $2 T_{\nu+1}\left(-\sqrt{\frac{(\nu+1)\left(1-\rho_{T}\right)}{1+\rho_{T}}}\right)$ \\
$\mathbf{C}_{\mathbf{C}}$ & $\frac{\theta_{C}}{\theta_{C}+2}$ & $2^{-1 / \theta_{C}}$ & - \\
$\mathbf{C}_{\mathbf{G}}$ & $1-\frac{1}{\theta_{G}}$ & - & $2-2^{1 / \theta_{G}}$ \\
$\mathbf{C}_{\mathbf{H}}$ & $\frac{\theta_{H}^{-1}}{\theta_{H}^{-1}+2}$ & - & $2^{-\theta_{H}}$ \\
\hline
\end{tabular}

\subsection{Modelos Cópula-GARCH}

Dizemos que um vetor aleatório $\boldsymbol{X}_{\boldsymbol{t}}=\left(X_{1 t}, \ldots, X_{n t}\right)$ tem distribuição dada por um modelo Cópula-GARCH se sua função de distribuição acumulada conjunta é dada por,

$$
F\left(\boldsymbol{x}_{\boldsymbol{t}}\right)=C\left(F_{X_{1 t}}\left(x_{1 t} \mid h_{x_{1 t}}\right), \ldots, F_{X_{n t}}\left(x_{n t} \mid h_{x_{n t}}\right)\right)
$$

na qual C é uma cópula n-dimensional, $F_{X_{i t}}$ é a i-ésima função de distribuição acumulada condicional da i-ésima variável aleatória $X_{i t}$, que que tem distribuição GARCH univariada, dada por (2.1). Em outras palavras, dizemos que um modelo multivariado é um modelo cópula-GARCH se ele tem estrutura de dependência dada por uma função de cópula e as distribuições marginais dadas por modelos GARCH univariados.

As densidades conjuntas foram obtidas para cada uma das funções de cópula (dadas por 2.5, 2.6, 2.7, 2.8 e 2.9), por meio da utilização da equação (1.3). Para tal, foi 
necessário determinar a função densidade de cópula, denotada por $c(\cdot)$, dada pela segunda derivada das funções de cópula, $\partial^{2} C(u, v) / \partial u \partial v$. Tomemos como exemplo o caso bivariado da Cópula Normal, que tem densidade de cópula dada por,

$$
c_{N}(u, v)=\frac{1}{\sqrt{1-\rho_{N}^{2}}} \cdot \exp \left\{\frac{\Phi^{-1}(u)^{2}+\Phi^{-1}(v)^{2}-2 \rho_{N} \Phi^{-1}(u) \Phi^{-1}(v)}{2\left(1-\rho_{N}^{2}\right)}+\frac{\Phi^{-1}(u)^{2} \Phi^{-1}(v)^{2}}{2}\right\} .
$$

Supondo que as distribuições marginais sejam dadas ambas por modelos GARCH com inovações $S T_{\nu_{x_{1}}}$ e $S T_{\nu_{x_{2}}}$, a densidade conjunta é dada por,

$$
\begin{aligned}
h\left(x_{t}, y_{t} \mid \boldsymbol{\theta}\right)= & \frac{1}{\sqrt{1-\rho_{N}^{2}}} \exp \left\{\frac{\Phi^{-1}(u)^{2}+\Phi^{-1}(v)^{2}-2 \rho_{N} \Phi^{-1}(u) \Phi^{-1}(v)}{2\left(1-\rho_{N}^{2}\right)}\right\} \\
& \exp \left\{\frac{\Phi^{-1}(u)^{2} \Phi^{-1}(v)^{2}}{2}\right\} \frac{\Gamma\left(\frac{\nu_{x_{1}}+1}{2}\right)}{\sqrt{\left(\nu_{x_{1}}-2\right) \pi h_{x_{1 t}}} \Gamma\left(\frac{\nu_{x_{1}}}{2}\right)}\left(1+\frac{x_{t}^{2}}{\left(\nu_{x_{1}}-2\right) h_{x_{1 t}}}\right)^{-\frac{\nu_{x_{1}}+1}{2}} \\
& \frac{\Gamma\left(\frac{\nu_{x_{2}}+1}{2}\right)}{\sqrt{\left(\nu_{x_{2}}-2\right) \pi h_{x_{2 t}}} \Gamma\left(\frac{\nu_{x_{2}}}{2}\right)}\left(1+\frac{x_{t}^{2}}{\left(\nu_{x_{2}}-2\right) h_{x_{2 t}}}\right)^{-\frac{\nu_{x_{2}}+1}{2}},
\end{aligned}
$$

na qual, $h_{x_{1 t}}$ e $h_{x_{2 t}}$ são dados pela equação (2.1), $u=F_{X_{1, t}}\left(x_{1 t} \mid \mathcal{F}_{x_{1}, t-1}, \theta_{x_{1}}\right), v=$ $F_{X_{2, t}}\left(x_{2 t} \mid \mathcal{F}_{x_{2}, t-1}, \theta_{x_{2}}\right)$ para $\mathcal{F}_{x_{1}, t-1}=x_{1, t-1}, \ldots, x_{1,1}, \mathcal{F}_{x_{2}, t-1}=x_{2, t-1}, \ldots, x_{2,1}$ e $\boldsymbol{\theta}=$ $\left(\theta_{C}, \theta_{x_{1}}, \theta_{x_{2}}\right)$ é o vetor de parâmetros da função de cópula e das distribuições marginais. Se as distribuições condicionais marginais seguem o modelo $\operatorname{GARCH}(1,1)$, que é o caso da presente dissertação, temos que, o vetor de parâmetros a ser estimado é $\boldsymbol{\theta}=\left(\omega_{x_{1}}, \alpha_{x_{1}}, \beta_{x_{1}}, \nu_{x_{1}}, \omega_{x_{2}}, \alpha_{x_{2}}, \beta_{x_{2}}, \nu_{x_{2}}, \rho_{N}\right)$. Deste modo, a função de verossimilhança é dada por,

$$
\mathcal{L}(\boldsymbol{\theta} ; \boldsymbol{x}, \boldsymbol{y})=\prod_{t=2}^{T} h\left(x_{t}, y_{t} \mid \boldsymbol{\theta}\right)
$$

A proxima seção apresenta as metodologias para estimação e interferência sobre os parâmetros em uma abordagem bayesiana. 


\subsection{Estimação e Inferência}

A literatura atual exibe uma grande quantidade algoritmos de estimação e inferência sobre parâmetros de modelos relacionados a cópulas. Na maioria dos trabalhos consultados, os parâmetros são estimados em uma abordagem clássica (por exemplo, Jondeau \& Rockinger, 2006; Liu \& Luger, 2009; Patton, 2006a; Abbara, 2009). Dentre estes trabalhos, muitos fazem uso de algoritmos de maximização por partes, como por exemplo o algoritmo "Inference Function for Margins" (IFM) utilizado em Filho (2010). Isto ocorre, pois a complexidade dos modelos utilizados pode dificultar o processo de otimização da função de log verossimilhança, em relação a todos os parâmetros simultaneamente (Joe \& Xu, 2008). Apesar dos estimadores obtidos desta forma serem consistentes e assintoticamente normais (da mesma forma que os obtidos pelo método convencional de máxima-verossimilhança), os obtidos pelos métodos de estimação em mais de um estágio podem ser assintoticamente menos eficientes, quando comparados aos do método de um estágio (Patton, 2006b).

Deste modo, utilizou-se neste trabalho, uma abordagem bayesiana, na qual os parâmetros foram estimados em conjunto e separadamente. Foram utilizados métodos baseados em simulações de Monte Carlo via cadeias de Markov (MCMC), mais especificamente o algoritmo de Metropolis-Hastings, proposto por Metropolis et al. (1953) (aplicações e maiores informações sobre o tema podem ser encontradas em Chib \& Greenberg, 1995, por exemplo.)

\subsubsection{O Algoritmo de Metropolis-Hastings}

Em um contexto bayesiano, são atribuídas funções de distribuição aos parâmetros, que são considerados variáveis aleatórias. Esta atribuição possibilita o acréscimo de informações a priori sobre os parâmetros do modelo estatístico, de acordo com o 
conhecimento de um especialista. Seja $p(\boldsymbol{\theta})$ a função de densidade de probabilidade conjunta do vetor de parâmetros $\boldsymbol{\theta}$ e $p(x)$ a função de densidade de probabilidade da v.a. $X$ relacionada a $\boldsymbol{\theta}$. Pelo teorema de Bayes temos,

$$
p(\boldsymbol{\theta} \mid x)=\frac{p(x, \boldsymbol{\theta})}{p(x)}=\frac{p(x \mid \boldsymbol{\theta}) p(\boldsymbol{\theta})}{p(x)}=\frac{p(x \mid \boldsymbol{\theta}) p(\boldsymbol{\theta})}{\int \ldots \int p(x, \boldsymbol{\theta}) d \boldsymbol{\theta}}
$$

na qual, $p(\boldsymbol{\theta} \mid x)$ é a função de densidade do conjunta do vetor de parâmetros $\boldsymbol{\theta}$, atualizada pelo valor observado de $X$, denominada distribuição a posteriori. Note que, como o termo $k=\int \ldots \int p(x, \boldsymbol{\theta}) d \boldsymbol{\theta}$ não depende de $\boldsymbol{\theta}$, ele pode ser visto como uma constante normalizadora. Dados valores observados da variável $X, \boldsymbol{x}=\left(x_{1}, \ldots, x_{n}\right)$, a função de verossimilhança $\mathcal{L}(\boldsymbol{\theta} ; \boldsymbol{x})$ é igual ao $\prod_{i=1}^{n} p\left(x_{i} \mid \boldsymbol{\theta}\right)$, logo,

$$
\pi(\boldsymbol{\theta})=p(\boldsymbol{\theta} \mid \boldsymbol{x}) \propto \mathcal{L}(\boldsymbol{\theta} ; \boldsymbol{x}) p(\boldsymbol{\theta}) .
$$

Obter uma amostra diretamente de $\pi(\boldsymbol{\theta})$ pode ser muito difícil, ou mesmo impossível em alguns casos. Uma alternativa que possibilita tal amostragem é o algoritmo de Metropolis-Hastings (M-H), que faz uso de uma função de distribuição auxiliar $q(\cdot, \cdot)$ para obter uma amostra da distribuição a posteriori. Para utilização do algoritmo de M-H, primeiramente deve-se atribuir valores inicias ao vetor de parâmetros $\boldsymbol{\theta}$, ou seja, definir $\boldsymbol{\theta}^{(0)}$. Então, para o índice de iteração $j=0, \ldots, N$, faça:

- Gere $\boldsymbol{\theta}^{\prime}$ de $q\left(\boldsymbol{\theta}^{(j)}, \cdot\right)$ e $u$ de $U \sim \mathcal{U}(0,1)$.

- Calcule a probabilidade de transição $\alpha\left(\boldsymbol{\theta}^{(j)}, \boldsymbol{\theta}^{\prime}\right)$, de $\boldsymbol{\theta}^{(j)}$ para $\boldsymbol{\theta}^{\prime}$,

$$
\begin{array}{rlrl}
\alpha\left(\boldsymbol{\theta}^{(j)}, \boldsymbol{\theta}^{\prime}\right) & =\min \left(\frac{\pi\left(\boldsymbol{\theta}^{\prime}\right) q\left(\boldsymbol{\theta}^{\prime}, \boldsymbol{\theta}^{(j)}\right)}{\pi\left(\boldsymbol{\theta}^{(j)}\right) q\left(\boldsymbol{\theta}^{(j)}, \boldsymbol{\theta}^{\prime}\right)}, 1\right), & \text { se } \pi\left(\boldsymbol{\theta}^{\prime}\right) q\left(\boldsymbol{\theta}^{\prime}, \boldsymbol{\theta}^{(j)}\right)>0 \\
& = & & \text { caso contrário }
\end{array}
$$

- Se $u<\alpha\left(\boldsymbol{\theta}^{(j)}, \boldsymbol{\theta}^{\prime}\right)$, faça $\boldsymbol{\theta}^{(j+1)}=\boldsymbol{\theta}^{\prime}$. Caso contrario, faça $\boldsymbol{\theta}^{(j+1)}=\boldsymbol{\theta}^{(j)}$. 
Os valores gerados $\left\{\boldsymbol{\theta}^{(1)}, \ldots, \boldsymbol{\theta}^{(n)}\right\}$ constituem uma amostra da distribuição de equilíbrio $\pi(\boldsymbol{\theta})$.

Neste trabalho, uma pequena modificação da distribuição normal multivariada, $\mathcal{N}_{m}(\boldsymbol{\mu}, \Sigma)$, foi utilizada como distribuição auxiliar. Essa pequena mudança foi necessária pois o domínio da $\mathcal{N}_{m}$ difere do espaço paramétrico $\Theta_{\boldsymbol{\theta}}$ dos modelos empregados. Dessa maneira, um novo vetor de parâmetros $\phi$ foi definido, de modo que, em um modelo com $m$ parâmetros, temos $\Theta_{\phi}=\mathbb{R}^{m}$ e existe uma transformação inversível $g: \Theta_{\boldsymbol{\theta}} \rightarrow \Theta_{\boldsymbol{\phi}}$, tal que, $g(\boldsymbol{\theta})=\boldsymbol{\phi}$. Assim, a função de distribuição auxiliar utilizada, é dada pela expressão,

$$
\begin{aligned}
q\left(\boldsymbol{\theta}, \boldsymbol{\theta}^{\prime}\right)= & \frac{1}{(2 \pi)^{m / 2} \sqrt{\operatorname{det}(\Sigma)}} \exp \left\{-\frac{1}{2}\left[g(\boldsymbol{\theta})-g\left(\boldsymbol{\theta}^{\prime}\right)\right]^{t} \Sigma^{-1}\left[g(\boldsymbol{\theta})-g\left(\boldsymbol{\theta}^{\prime}\right)\right]\right\} \\
& \prod_{i=1}^{m} g_{i}^{\prime}\left(\theta_{i}\right),
\end{aligned}
$$

na qual, $g_{i}^{\prime}\left(\theta_{i}\right)$ é a primeira derivada da i-ésima coordenada da transformação $g(\cdot)$ em relação à i-ésima coordenada do vetor $\boldsymbol{\theta}$ e $\Sigma$ é a matriz de covariância, que pode ser estimada a partir de uma amostra piloto. Note que, deste modo, as distribuições a priori podem ser atribuídas diretamente aos parâmetros do vetor $\boldsymbol{\theta}$ e a probabilidade de transição (equação 2.10) é calculada pela expressão que segue,

$$
\begin{array}{rlrl}
\alpha\left(\boldsymbol{\theta}^{(j)}, \boldsymbol{\theta}^{\prime}\right) & =\min \left(\frac{\pi\left(\boldsymbol{\theta}^{\prime}\right) \prod_{i=1}^{m} g_{i}^{\prime}\left(\theta_{i}^{\prime}\right)}{\pi\left(\boldsymbol{\theta}^{(j)}\right) \prod_{i=1}^{m} g_{i}^{\prime}\left(\theta_{i}^{(j)}\right)}, 1\right), & \text { se } \pi\left(\boldsymbol{\theta}^{(j)}\right) \prod_{i=1}^{m} g_{i}^{\prime}\left(\theta_{i}^{(j)}\right)>0 \\
& = & 1, & \text { caso contrário. }
\end{array}
$$

Durante a implementação das rotinas, a estimação dos parâmetros foi testada de varias formas, isto é, foram considerados diferentes blocos de parâmetros para o M-H (ver Hastings, 1970). Entretanto, o melhor resultado, no sentido de menor autocorrelação entre os valores da amostra da posteriori e menor tempo computacional, foi 
obtido para o caso em que os parâmetros eram gerados todos de uma vez, logo, todas as simulações foram realizadas deste modo.

\subsubsection{Estimação em Um e Dois Passos}

A estimação em um passo ocorreu de forma usual, pela utilização do algoritmo de Metropolis Hastings supracitado. Nela, todos os parâmetros desconhecidos foram estimados em conjunto. Já a estimação em dois passos foi esquematizada da mesma forma que em Ausin \& Lopes (2010), descrita da seguinte maneira,

Passo 1: Estimar os parâmetros das marginais $\theta_{x 1}$, e $\theta_{x 2}$

Passo 2: Dados $\tilde{\theta}_{x 1}$ e $\tilde{\theta}_{x 2}$, estimar os parâmetros da cópula $\theta_{C}$, na pseudo-verossimilhança (exemplificada pela equação 2.11).

Supondo que as marginais sigam o modelo $\operatorname{GARCH}(1,1)$ com inovações $S T$, a verossimilhança da distribuição marginal $X_{1}$, é dada por,

$$
\mathcal{L}\left(\theta_{x_{1}} \mid \boldsymbol{x}_{\mathbf{1}}\right) \propto \prod_{t=1}^{T} \frac{\Gamma\left(\frac{\nu_{x_{1}}+1}{2}\right)}{\sqrt{\left(\nu_{x_{1}}-2\right) h_{t}} \Gamma\left(\frac{\nu_{x_{1}}}{2}\right)}\left(1+\frac{x_{1}^{2}}{\left(\nu_{x_{1}}-2\right) h_{t}}\right)^{-\frac{\nu+1}{2}} .
$$

Seja $N$ o número de iterações necessárias para a convergência do algoritmo de estimação dos parâmetros das marginais, desconsiderando as iterações de aquecimento, temos que, para cada elemento da amostra de Monte Carlo obtém-se um conjunto de resíduos, isto é,

$$
\alpha_{x_{1}, 0}^{(n)}, \alpha_{x_{1}, 1}^{(n)}, \beta_{x_{1}}^{(n)}, \nu_{x_{1}}^{(n)} \Rightarrow \varepsilon_{t}^{(n)}=\frac{x_{1 t}}{\sqrt{h_{t}^{(n)}}}, \quad \text { para } t=1,2, \ldots, T
$$

As estimativas pontuais dos parâmetros e do resíduo no tempo $t\left(\varepsilon_{x_{1}, t}\right)$, foram 
obtidas pela média à posteriori, que é dada por,

$$
\begin{gathered}
\tilde{\alpha}_{x_{1}, 0}=\frac{1}{N} \sum_{n=1}^{N} \alpha_{x_{1}, 0}^{(n)}, \quad \tilde{\alpha}_{x_{1}, 1}=\frac{1}{N} \sum_{n=1}^{N} \alpha_{x_{1}, 1}^{(n)}, \quad \tilde{\beta}_{x_{1}}=\frac{1}{N} \sum_{n=1}^{N} \beta_{x_{1}}^{(n)}, \\
\tilde{\nu}_{x_{1}}=\frac{1}{N} \sum_{n=1}^{N} \nu_{x_{1}}^{(n)} \quad \text { e } \quad \tilde{\varepsilon}_{x_{1}, t}=\frac{1}{N} \sum_{n=1}^{N} \varepsilon_{x_{1}, t}^{(n)}
\end{gathered}
$$

A estimativa dos resíduos é utilizada para obtenção dos valores $u_{t}$ para marginal $X_{1 t}$ e $v_{t}$ para marginal $X_{2 t}$, que são utilizados, juntamente com a estimativa dos parâmetros, na pseudo-verossimilhança. Ou seja, para $S T_{\nu}(\cdot)$ a função de distribuição acumulada da $S T$ com graus de liberdade iguais à $\nu$, temos,

$$
\tilde{u}_{t}=S T_{\tilde{\nu}_{x_{1}}}\left(\tilde{\varepsilon}_{x_{1}, t}\right) \text { e } \quad \tilde{v}_{t}=S T_{\tilde{\nu}_{x_{2}}}\left(\tilde{\varepsilon}_{x_{2}, t}\right)
$$

A pseudo-verossimilhança é então dada por,

$$
\prod_{t=1}^{T} \frac{1}{\sqrt{1-\rho_{N}^{2}}} \cdot \exp \left\{\frac{\Phi^{-1}\left(\tilde{u}_{t}\right)^{2}+\Phi^{-1}\left(\tilde{v}_{t}\right)^{2}-2 \rho_{N} \Phi^{-1}\left(\tilde{u}_{t}\right) \Phi^{-1}\left(\tilde{v}_{t}\right)}{2\left(1-\rho_{N}^{2}\right)}+\frac{\Phi^{-1}\left(\tilde{u}_{t}\right)^{2} \Phi^{-1}\left(\tilde{v}_{t}\right)^{2}}{2}\right\} .
$$

Deve-se, por fim, construir outra cadeia de Markov baseada na pseudoverossimilhança para gerar amostras da posteriori de $\rho_{N}$.

É válido lembrar que os métodos MCMC fornecem uma amostra da distribuição a posteriori, ou seja, além das estimativas pontuais dos parâmetros, outras características são obtidas de forma imediata, como variância, correlações e intervalos de credibilidade.

\subsection{Geração de séries aleatórias}

Esta seção tem como objetivo apresentar o algoritmo utilizado no capítulo 4, para gerar séries aleatórias dos modelos cópula-GARCH. Ele foi elaborado para o 
caso bivariado, mas pode ser generalizado, para dimensões superiores a dois, sem grandes dificuldades. As séries geradas tem distribuição GARCH(1,1) com erros SST e estrutura de dependência especificada por uma função de cópula.

Passo 1: Especificar a função de cópula bivariada a ser utilizada $(C$.) e fixar o parâmetro da cópula segundo um valor de $\tau$. Fixar também os parâmetros das distribuições marginais $\left(\omega_{x_{1}}, \alpha_{x_{1}}, \beta_{x_{1}}, \nu_{x_{1}}, \gamma_{x_{1}}, \omega_{x_{2}}, \alpha_{x_{2}}, \beta_{x_{2}}, \nu_{x_{2}}, \gamma_{x_{2}}\right)$.

Passo 2: Gerar uma amostra aleatória $\left(\left(u_{1}, v_{1}\right), \ldots,\left(u_{n}, v_{n}\right)\right)$ da cópula previamente especificada. Informações sobre como gerar amostras aleatórias das cópula podem ser encontradas em Nelsen (2006). Neste trabalho as amostras foram geradas pela utilização do pacote "copula" (Yan, 2007; Kojadinovic \& Yan, 2010) do software R Development Core Team (2010).

Passo 3: Atribua um valor inicial a primeira coordenada dos vetores de volatilidade das distribuições marginais, $h_{x_{1}, 1}$ e $h_{x_{2}, 1}$ e calcule a primeira coordenada dos vetores $\boldsymbol{x}_{\mathbf{1}}=\left(x_{1,1}, \ldots, x_{1, t}\right)$ e $\boldsymbol{x}_{\mathbf{2}}=\left(x_{2,1}, \ldots, x_{2, t}\right)$ da seguinte maneira,

$$
\begin{aligned}
& x_{1,1}=S S T^{(-1)}\left(u_{1} \mid \nu_{x_{1}}, \gamma_{x_{1}}\right) \sqrt{h_{x_{1}, 1}}, \\
& x_{2,1}=S S T^{(-1)}\left(v_{1} \mid \nu_{x_{2}}, \gamma_{x_{2}}\right) \sqrt{h_{x_{2}, 1}},
\end{aligned}
$$

na qual $S S T^{(-1)}(\cdot)$ é a função quantil de uma v.a. com distribuição $S S T$ (uma implementação desta função pode ser encontrada no pacote fGarch: RmetricsAutoregressive Conditional Heteroskedastic Modelling).

Passo 4: Para $j=2, \ldots n$ faça:

$$
\begin{aligned}
& h_{x_{1}, j}=\omega_{x_{1}}+\alpha_{x_{1}} x_{1, j-1}^{2}+\beta_{x_{1}} h_{x_{1}, j-1} \\
& x_{1, j}=S S T^{(-1)}\left(u_{j} \mid \nu_{x_{1}}, \gamma_{x_{1}}\right) \sqrt{h_{x_{1}, j}} \\
& h_{x_{2}, j}=\omega_{x_{2}}+\alpha_{x_{2}} x_{2, j-1}^{2}+\beta_{x_{2}} h_{x_{2}, j-1} \\
& x_{2, j}=S S T^{(-1)}\left(v_{j} \mid \nu_{x_{2}}, \gamma_{x_{2}}\right) \sqrt{h_{x_{2}, j}}
\end{aligned}
$$


Os vetores $\boldsymbol{x}_{1}$ e $\boldsymbol{x}_{\mathbf{2}}$ constituem uma série bivariada do modelo cópula-GARCH em questão. As primeiras observações devem ser descartadas, pois isto permite minimizar efeito dos valores iniciais atribuídos à primeira coordenada dos vetores de volatilidade.

Uma extensão natural da teoria das cópulas, apresentada neste capítulo, é fazer com que os parâmetros da função de cópula variem ao longo do tempo, para capturar eventuais mudanças na estrutura de dependência. O próximo capítulo apresenta alguns conceitos relacionados à esta extensão. 


\section{Capítulo 3}

\section{Modelos com Dependência Variante}

\section{no Tempo}

Assim como a volatilidade é um fato estilizado em séries de retornos do mercado financeiro, há evidência empírica nos trabalhos recentes de que a estrutura e o grau de dependência entre retornos podem mudar ao longo do tempo (por exemplo, Arakelian \& Dellaportas, 2012; Ausin \& Lopes, 2010; Rodriguez, 2007; Zhang \& Guegan, 2008). Uma alternativa para dimensionar essas possíveis mudanças na estrutura de dependência é propor modelos, nos quais os parâmetros da função de cópula (parâmetros que quantificam o grau de dependência entre as distribuições marginais) se alterem ao longo do tempo, seguindo uma equação de evolução específica.

Se os parâmetros de cópula variam ao longo tempo e se estes dependem de seus valores defasados ou de valores defasados das distribuições marginais, a densidade condicional conjunta é determinada pela utilização da versão condicional do Teorema 1.1 proposta por Patton (2006a) e apresentada abaixo.

Teorema 3.1. (Teorema de Sklar - versão condicional) Sejam $X_{1}\left|W, X_{2}\right| W, \ldots$, $X_{n} \mid W$ variáveis aleatórias com distribuição condicional acumulada $F_{1}, F_{2}, \ldots F_{n}$ res- 
pectivamente e distribuição acumulada conjunta $H$ de $\mathbf{X} \mid \mathbf{W}$ para $\mathbf{X}=\left(X_{1}, X_{2}, \ldots, X_{n}\right)$ e $W$ com suporte em $\Omega$. Então existe uma função $C$ tal que,

$$
H\left(x_{1}, x_{2}, \ldots, x_{n} \mid W\right)=C\left(F_{1}\left(x_{1} \mid W\right), F_{2}\left(x_{2} \mid W\right), \ldots, F_{n}\left(x_{n} \mid W\right) \mid W\right)
$$

Para todo $x_{1}, x_{2}, \ldots, x_{n} \in \overline{\mathbb{R}}$ e todo $w \in \Omega$. Se $F_{1}, F_{2}, \ldots, F_{n}$ são continuas, $C$ é única, caso contrario $C$ é determinada somente em $\operatorname{Im} F_{1} \times \ldots \times \operatorname{Im} F_{n}$. Reciprocamente se $C$ é uma n-cópula e $F_{1}, F_{2}, \ldots, F_{n}$ são distribuições acumuladas, então a função $H$ é uma distribuição conjunta n-dimensional com marginais $F_{1}, F_{2}, \ldots, F_{n}$.

De forma análoga, a versão condicional do Corolário 1.1 foi obtida pelo autor.

Corolário 3.1. Seja $H$ uma função de distribuição acumulada conjunta condicional com marginais condicionais $F_{1}, F_{2}, \ldots, F_{n}$ continuas e função de cópula $C$. Então, para todo $\mathbf{u}=\left(u_{1}, u_{2}, \ldots, u_{n}\right) \in[0,1]^{n}$

$$
C\left(u_{1}, u_{2}, \ldots, u_{n}\right)=H\left(F_{1}^{-1}\left(u_{1} \mid W\right), F_{2}^{-1}\left(u_{2} \mid W\right), \ldots, F_{n}^{-1}\left(u_{n} \mid W\right)\right)
$$

O conjunto de informações $W$ precisa ser o mesmo para a função de cópula e para as distribuições marginais. Caso contrario $C$, não satisfaz a definição de cópula, podendo ser tratada como uma pseudo-cópula que tem suas propriedades particulares (Fermanian \& Scaillet, 2004).

Uma alternativa proposta por Patton (2006a) e utilizada por Abbara (2009), para o caso em que as marginais são condicionadas à diferentes variáveis aleatórias, é verificar a validade das igualdades (3.2) e (3.3). Este procedimento é realizado por meio de uma regressão dos resíduos do modelo marginal de $X_{1, t}$ ajustado, contra a primeira defasagem dos retornos $X_{2, t}$ e analogamente para os resíduos do modelo de $X_{2, t}$. O mesmo deve ser feito para o quadrado dos retorno. Consideram-se válidas as igualdades 
se a hipótese de que o coeficiente regressor é igual a zero não for rejeitada, em ambos os casos.

$$
\begin{aligned}
& F_{x_{1}}\left(x_{1, t} \mid \mathcal{F}_{x_{1}, t-1}\right)=F_{x_{1}}\left(x_{1, t} \mid \mathcal{F}_{x_{1}, t-1}, \mathcal{F}_{x_{2}, t-1}\right), \\
& F_{x_{2}}\left(x_{2, t} \mid \mathcal{F}_{x_{2}, t-1}\right)=F_{x_{2}}\left(x_{2, t} \mid \mathcal{F}_{x_{1}, t-1}, \mathcal{F}_{x_{2}, t-1}\right),
\end{aligned}
$$

nas quais $\mathcal{F}_{x_{1}, t-1}=x_{1, t-1}, \ldots, x_{1,1}$ e $\mathcal{F}_{x_{2}, t-1}=x_{2, t-1}, \ldots, x_{2,1}$.

Satisfeitas as igualdades (3.2) e (3.3), para o caso bidimensional, se $F_{x_{1}}$ e $F_{x_{2}}$ são diferenciáveis e $C$ é diferenciável de segunda ordem, podemos obter de (3.1) a densidade condicional conjunta $h$

$$
\begin{gathered}
h\left(x_{1, t}, x_{2, t} \mid W\right)=\frac{\partial^{2} H\left(x_{1, t}, x_{2, t} \mid W\right)}{\partial x_{1, t} \partial x_{2, t}}=\frac{\partial F_{x_{1}}\left(x_{1, t} \mid \mathcal{F}_{x_{1, t}, t-1}\right)}{\partial x_{1, t}} \frac{\partial F_{x_{2}}\left(x_{2, t} \mid \mathcal{F}_{x_{2}, t-1}\right)}{\partial x_{2, t}} \\
\frac{\partial^{2} C\left(F_{x}\left(x_{1, t} \mid \mathcal{F}_{x_{1}, t-1}\right), F_{x_{2}}\left(x_{2, t} \mid \mathcal{F}_{x_{2}, t-1}\right) \mid W\right)}{\partial u \partial v} \\
h\left(x_{1, t}, x_{2, t} \mid W\right)=f_{x_{1}}\left(x_{1, t} \mid \mathcal{F}_{x_{1}, t-1}\right) f_{x_{2}}\left(x_{2, t} \mid \mathcal{F}_{x_{2}, t-1}\right) c(u, v \mid W)
\end{gathered}
$$

para $u=F_{x_{1}}\left(x_{1, t} \mid \mathcal{F}_{x_{1}, t-1}\right), v=F_{x_{2}}\left(x_{2, t} \mid \mathcal{F}_{x_{2}, t-1}\right)$ e $W=\left(\mathcal{F}_{x_{1}, t-1}, \mathcal{F}_{x_{2}, t-1}\right)$.

Desta forma, a função log-verossimilhança de uma amostra $\left\{x_{1, t}, x_{2, t}\right\}_{t=1}^{T}$, com vetor de parâmetros $\boldsymbol{\theta}=\left(\theta_{C}, \theta_{X_{1}}, \theta_{X_{2}}\right)$, com $\theta_{X_{1}}$ e $\theta_{X_{2}}$ parâmetros das distribuições marginais e $\theta_{C}$ parâmetros do modelo de cópula, é dada por,

$$
\begin{aligned}
\ell(\boldsymbol{\theta})= & \sum_{t=1}^{T} \log c\left(F_{x_{1}}\left(x_{1, t} \mid \mathcal{F}_{x_{1}, t-1}, \theta_{X_{1}}\right), F_{x_{2}}\left(x_{2, t} \mid \mathcal{F}_{x_{2}, t-1}, \theta_{X_{2}}\right) \mid W, \theta_{C}\right) \\
& +\sum_{t=1}^{T} \log f_{x_{1}}\left(x_{1, t} \mid \mathcal{F}_{x_{1}, t-1}, \theta_{X_{1}}\right)+\log f_{x_{2}}\left(y_{2, t} \mid \mathcal{F}_{x_{2}, t-1}, \theta_{X_{2}}\right) .
\end{aligned}
$$

Neste trabalho, as variações da estrutura de dependência no tempo foram capturadas pelo uso da metodologia desenvolvida por Patton (2006a). Nesta metodologia, o parâmetro, em um determinado instante no tempo, depende do valor que ele assumiu em suas defasagens e de valores provenientes de defasagens das 
observações das distribuições marginais.

Para a função de cópula Normal a equação de evolução do parâmetro $\rho_{t}$, indexado pelo tempo, foi especificada da seguinte maneira,

$$
\rho_{t}=\Lambda\left(\omega_{N}+\beta_{N} \rho_{t-1}+\alpha_{N} \cdot \frac{1}{d} \sum_{j=1}^{d} \Phi^{-1}\left(u_{t-j}\right) . \Phi^{-1}\left(v_{t-j}\right)\right),
$$

na qual, $\omega_{N}, \beta_{N}$ e $\alpha_{N}$ são parâmetros, $d$ é o numero de defasagens levado em consideração e $\Phi^{-1}($.$) é a função quantil da normal padrão. O parâmetro \rho_{t}$ pode ser mantido no intervalo $[-1,1]$ pela transformação $\Lambda(x)=\frac{1-e^{-x}}{1+e^{-x}}$. Assim como no trabalho de Abbara (2009), foram testados vários valores para $d$, inclusive $d=10$ (valor utilizado por Patton, 2006a), porém, os melhores resultados (menor variabilidade do parâmetro das cópulas) foram obtidos para $d=1$.

A cópula t de Student tem equação de evolução para $\rho_{t}$ dada por,

$$
\rho_{t}=\Lambda\left(\omega_{T}+\beta_{T} \rho_{t-1}+\alpha_{T} \cdot \frac{1}{d} \sum_{j=1}^{d} T_{\nu}^{-1}\left(u_{t-j}\right) \cdot T_{\nu}^{-1}\left(v_{t-j}\right)\right)
$$

na qual, $T_{\nu}^{-1}($.$) é a função quantil da distribuição t$ com $\nu$ graus de liberdade. $\mathrm{O}$ parâmetro $\rho_{t}$ pode ser mantido no intervalo $[-1,1]$ pela mesma transformação utilizada na copula $C_{N}$.

Nas demais cópulas (Clayton, Gumbel e Heavy Tail), foram utilizadas equações de evolução similares. Porém, estas cópulas foram reparametrizadas nas medidas de dependência de cauda, especificadas na tabela 2.1. A cópula Clayton, por exemplo, é dada pela equação,

$$
C_{C}\left(u_{t}, v_{t} \mid \theta_{C, t}\right)=\left(u_{t}^{-\theta_{C, t}}+v_{t}^{-\theta_{C, t}}-1\right)^{-\frac{1}{\theta_{C, t}}}
$$


na qual,

$$
\theta_{C, t}=-\frac{\ln 2}{\ln \lambda_{C L, t}}
$$

para $\lambda_{C L, t}$ variando no tempo segundo a equação de evolução,

$$
\lambda_{C L, t}=\Lambda\left(\omega_{C}+\beta_{C} \lambda_{C L, t-1}+\alpha_{C} \cdot \frac{1}{d} \sum_{j=1}^{d}\left|u_{t-j}-v_{t-j}\right|\right) .
$$

Neste caso, a transformação utilizada para manter a equação de evolução no intervalo de definição das medidas de dependência nas caudas $(0,1]$ foi $\Lambda(x)=$ $1 /(1+\exp (-x))$. As equações de evolução para as medidas de dependência nas caudas das cópulas Gumbel e Heavy Tail foram obtidas de forma análoga.

A reparametrização nas medidas de dependência nas caudas foi realizada pois esta facilita a interpretação dos gráficos de variação de dependência no tempo. Além disso, a reparametrização permitiu eliminar problemas numéricos do algoritmo, o que facilitou a implementação das rotinas MCMC.

Para determinar a densidade condicional conjunta deve-se utilizar a equação (3.4). Os parâmetros para estes modelos são estimados de maneira análoga à apresentada no capítulo 2 para cópulas estáticas.

Para o exemplo apresentado nos capítulos anteriores (o da cópula Normal com distribuições marginais $\operatorname{GARCH}(1,1)$ com erros $S T)$ o vetor de parâmetros a ser estimado é $\boldsymbol{\theta}=\left(\omega_{x_{1}}, \alpha_{x_{1}}, \beta_{x_{1}}, \nu_{x_{1}}, \omega_{x_{2}}, \alpha_{x_{2}}, \beta_{x_{2}}, \nu_{x_{2}}, \omega_{N}, \alpha_{N}, \beta_{N}\right)$ e a função de 
verossimilhança é dada por,

$$
\begin{aligned}
\mathcal{L}\left(\boldsymbol{\theta} \mid \boldsymbol{x}_{\mathbf{1}}, \boldsymbol{x}_{\mathbf{2}}\right)= & \prod_{t=k+1}^{T} \frac{1}{\sqrt{1-\rho_{t}^{2}}} \exp \left\{\frac{\Phi^{-1}(u)^{2}+\Phi^{-1}(v)^{2}-2 \rho_{t} \Phi^{-1}(u) \Phi^{-1}(v)^{2}}{2\left(1-\rho_{t}^{2}\right)}\right\} \\
& \exp \left\{\frac{\Phi^{-1}(u)^{2} \Phi^{-1}(v)^{2}}{2}\right\} \frac{\Gamma\left(\frac{\nu_{x_{1}}+1}{2}\right)}{\sqrt{\left(\nu_{x_{1}}-2\right) h_{x_{1}, t}} \Gamma\left(\frac{\left.\nu_{x_{1}}\right)}{2}\right)}\left(1+\frac{x_{1 t}^{2}}{\left(\nu_{x_{1}}-2\right) h_{x_{1}, t}}\right)^{-\frac{\nu_{x_{1}+1}}{2}} \\
& \frac{\Gamma\left(\frac{\nu_{x_{2}}+1}{2}\right)}{\sqrt{\left(\nu_{x_{2}}-2\right) h_{x_{2}, t}} \Gamma\left(\frac{\nu_{x_{2}}}{2}\right)}\left(1+\frac{x_{2 t}^{2}}{\left(\nu_{x_{2}}-2\right) h_{x_{2}, t}}\right)^{-\frac{\nu_{x_{2}}+1}{2}}
\end{aligned}
$$

para $k=\max (p, q, d), p, q$ argumentos do modelo $\operatorname{GARCH}(\mathrm{p}, \mathrm{q})$ e $\rho_{t}$ dado pela equação de evolução (3.5).

No capítulo que segue, são apresentados os resultados obtidos por estudos de simulação relacionados à seleção de modelos. 


\section{Capítulo 4}

\section{Estudos de simulação}

Conforme mencionado no inicio da seção 2.4, a literatura sugere que, do ponto de vista Bayesiano, os parâmetros sejam estimados em conjunto, para que seja levada em consideração a estrutura de dependência entre eles.

O nosso objetivo neste capítulo foi verificar como a seleção de modelos cópulaGARCH é influenciada pelos métodos de estimação (em conjunto e separadamente), tamanho das séries e valores dos parâmetros das funções de cópula. Esta verificação foi feita por meio de estudos de simulação similares aos apresentados por Silva \& Lopes (2008).

Todas os códigos utilizados nas simulações foram implementadas no software $\mathrm{C}$ e executadas no cluster Puma do Laboratório de Computação Científica Avançada - LCCA da USP. Algumas funções da biblioteca GSL (Galassi et al., 2010) foram utilizadas na implementação dos códigos. 


\subsection{Especificação dos modelos}

Nos estudos foram utilizados dez modelos bivariados. Estes modelos foram obtidos por meio da combinação das estrutura de dependência, especificadas por cada uma das cinco funções de cópulas estáticas apresentadas na seção 2.2 (equações 2.5 à 2.9), e de dois modelos marginais GARCH(1,1), os com erros ST e os com erro SST. Em seguida, foram geradas 500 réplicas de séries bivariadas de tamanhos 200, 500, e 1000 para cada um dos modelos, com dois valores para os parâmetros das cópulas, determinados pelos valores do Tau de Kendall $(\tau)$ iguais a $1 / 3$ e 2/3. As séries foram geradas pela aplicação do algoritmo apresentado na seção 2.5 .

Os modelos GARCH com erros $S S T$, utilizados para gerar as séries, foram especificados da seguinte maneira:

$$
\begin{aligned}
X_{1 t} & =\sqrt{h_{x_{1}, t}} \varepsilon_{x_{1} t}, & X_{2 t} & =\sqrt{h_{x_{2}, t}} \varepsilon_{x_{2} t}, \\
h_{x_{1}, t} & =0.0001+0.08 X_{1 t-1}^{2}+0.9 h_{x_{1}, t-1}, & & h_{x_{2}, t}=0.0003+0.11 X_{2 t-1}^{2}+0.88 h_{x_{2}, t-1}, \\
\varepsilon_{x_{1} t} & \sim S S T\left(\nu_{x_{1}}=9, \gamma_{x_{1}}=0.96\right) ; & \varepsilon_{x_{2} t} & \sim S S T\left(\nu_{x_{2}}=8, \gamma_{x_{2}}=0.98\right) .
\end{aligned}
$$

Os mesmos parâmetros foram utilizados para gerar as séries dos modelos com erros $S T$, com exceção dos parâmetros de assimetria que foram fixados $\gamma_{x_{1}}=1, \gamma_{x_{2}}=1$. $\AA$ cada uma das réplicas geradas, considerando as distribuições marginais com erros $S T$, foram ajustados os cinco modelos bivariados obtidos com erros $S T$ nas distribuições marginais. Da mesma maneira, às réplicas com erros $S S T$, foram ajustados somente os cinco modelos com erros SST. Os algoritmos de estimação foram executados por 30.000 iterações e os valores obtidos nas 20.000 primeiras foram consideradas como uma amostra de aquecimento.

As distribuições a priori utilizadas foram consideras independentes e normais, truncadas no intervalo de definição de cada parâmetro, $N^{T}\left(\mu, \sigma^{2}\right)$. Ou seja, aos parâmetros 
$\omega_{x_{1}}, \alpha_{x_{1}}, \beta_{x_{1}}, \omega_{x_{2}}, \alpha_{x_{2}}, \beta_{x_{2}}, \rho_{N}, \rho_{T}, \theta_{C}, \theta_{G}, \theta_{H}$ foram atribuídas distribuições a priori $N^{T}(0,100)$. Aos parâmetros $\gamma_{x_{1}}, \gamma_{x_{2}}$, distribuições a priori $N^{T}\left(0,0.64^{-1}\right)$ e aos parâmetros $\nu_{x_{1}}$ e $\nu_{x_{2}}$, distribuições a priori $N^{T}(8,30)$. As distribuições a priori atribuídas aos parâmetros de assimetria foram especificadas desta maneira pois alocam probabilidades similares para assimetrias positiva e negativa, sendo deste modo consideradas pouco informativas (Fernandez \& Steel, 1998). A mesma metodologia foi utilizada para definir as distribuições à priori para os graus de liberdade.

Distribuições à priori normais truncadas com variância grande foram atribuídas aos demais parâmetros pois estas não incluem grande quantidade de informação no modelo. Para comprovar este fato foram realizados testes nos quais as distribuições à priori normais truncadas (em um intervalo fechado) foram substituídas por distribuições uniformes. Observou-se que as estimativas obtidas com distribuições à priori uniformes eram muito semelhantes quando comparadas com as obtidas por meio do uso das normais truncadas. Deste modo, as distribuições normais truncadas foram mantidas pois seus parâmetros facilitam a inclusão de informação.

\subsection{Critérios para seleção de modelos}

Os modelos foram selecionados segundo os critérios determinados pelo o valor esperado do Akaike information criterion (Akaike, 1973) o (EAIC) e o valor esperado do Bayesian information criterion (Schwarz, 1978) o (EBIC). Note que, para este estudo de simulações, os critérios EAIC e EBIC produzem o mesmo resultado, pois, os modelos envolvidos em cada seleção tem a mesma quantidade de parâmetros. Os resultados obtidos por estes critérios foram comparados com os obtidos pelo deviance 
information criterion (DIC). Estes critérios são dados por,

$$
\begin{aligned}
& E A I C=2 E\left[D\left(\boldsymbol{\theta}_{\boldsymbol{M}}\right)\right]+2 n p_{M}, \\
& E B I C=2 E\left[D\left(\boldsymbol{\theta}_{\boldsymbol{M}}\right)\right]+\ln (n) n p_{M}, \\
& D I C=2 E\left[D\left(\boldsymbol{\theta}_{\boldsymbol{M}}\right)\right]-D\left(E\left[\boldsymbol{\theta}_{\boldsymbol{M}}\right]\right),
\end{aligned}
$$

nos quais,

$$
D\left(\boldsymbol{\theta}_{M}\right)=-2 \ln \mathcal{L}\left(\boldsymbol{\theta}_{M}\right)
$$

$\boldsymbol{\theta}_{M}$ é o vetor de parâmetros do modelo $M, n p_{M}$ é o número de parâmetros do modelo $M$ e $\mathcal{L}\left(\boldsymbol{\theta}_{M}\right)$ é a função de verossimilhança avaliada em $\boldsymbol{\theta}_{M}$.

É valido lembrar que ao utilizar as medidas supracitadas em conjunto com os algoritmos MCMC, estas são facilmente aproximadas pois tem-se uma amostra da distribuição à posteriori dos parâmetros. Além disso, estas medidas são invariantes a transformações monótonas crescentes nas distribuições marginais. Por exemplo, se um modelo de cópula $C$ com distribuições marginais normais é selecionado como o de melhor ajuste para um conjunto de dados, o mesmo modelo de cópula será selecionado ao substituir as distribuições marginais por log-normais (Silva \& Lopes, 2008). Maiores informações sobre o DIC e sobre as demais medidas podem ser encontradas em Spiegelhalter et al. (2002).

\subsection{Resultados e Conclusões}

Nesta seção são apresentadas as tabelas que contém as proporções de seleção do modelo correto (aquele que foi utilizado para gerar a série), segundo os critérios EAIC/EBIC e DIC, gráficos com a proporção de seleção de cada modelo para cada conjunto de réplicas e algumas considerações sobre os resultados. Nos gráficos, as colunas representam o modelo de cópula utilizado para gerar as réplicas e as cores representam a proporção de seleção de cada modelo segundo as legendas. 


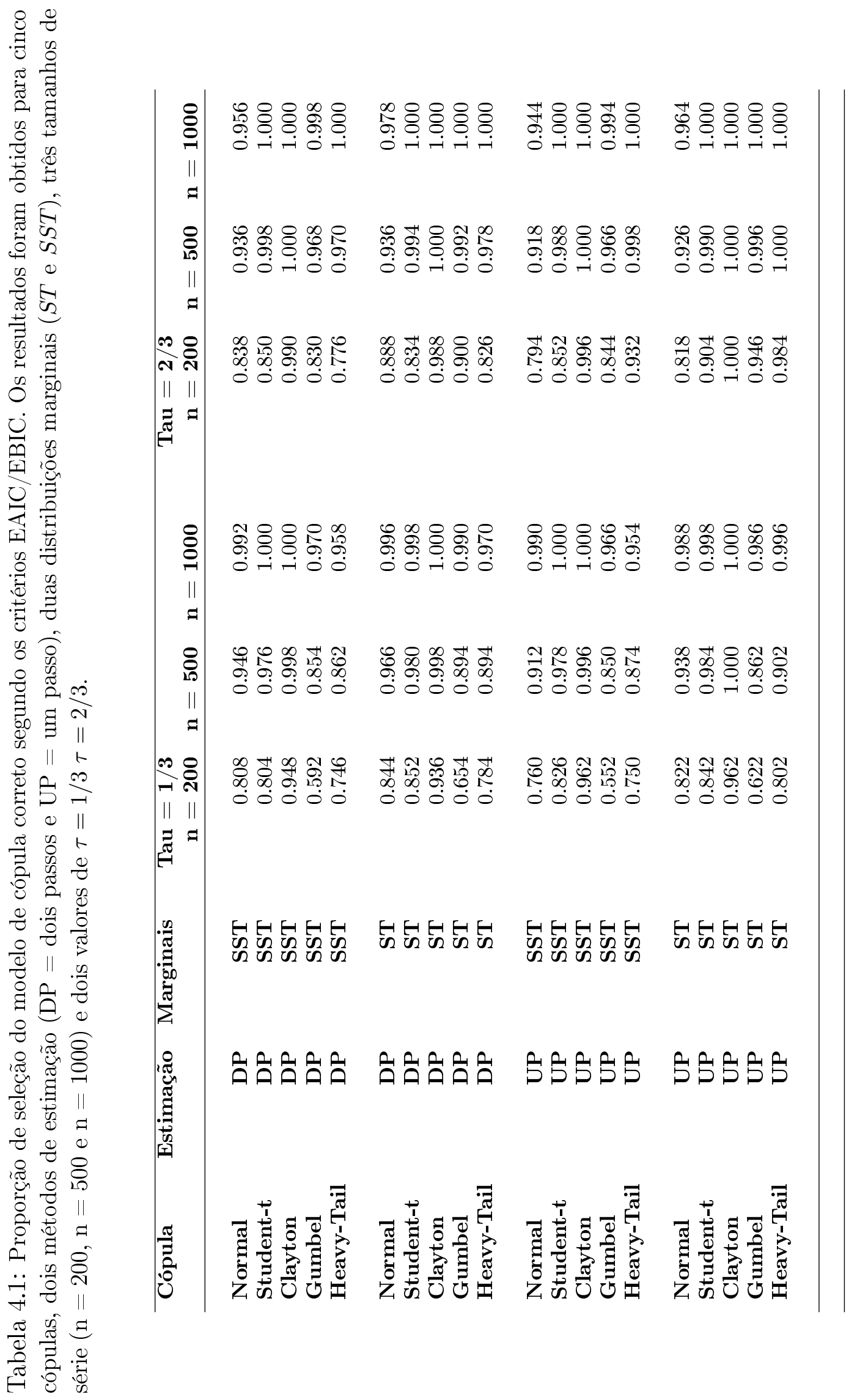




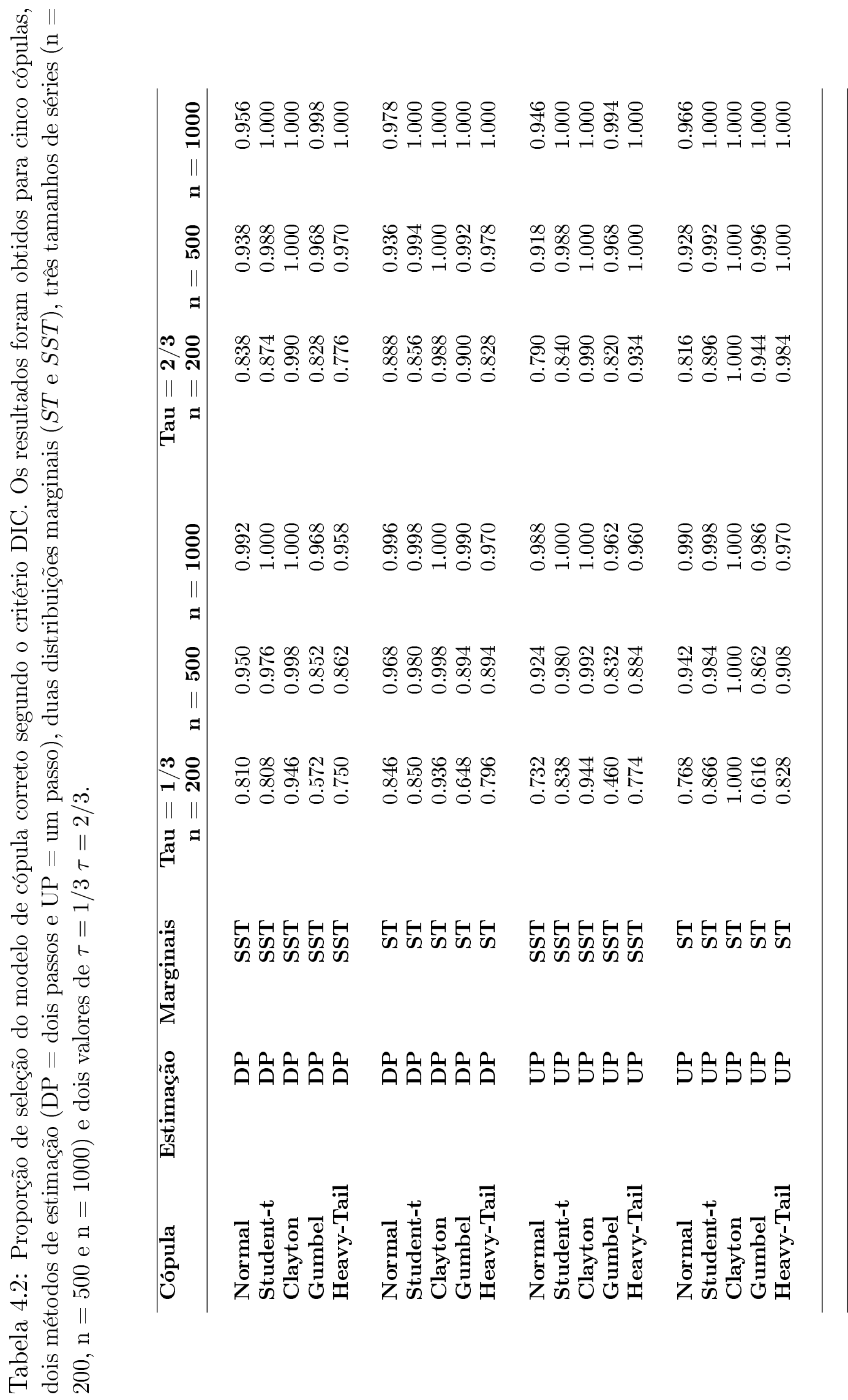



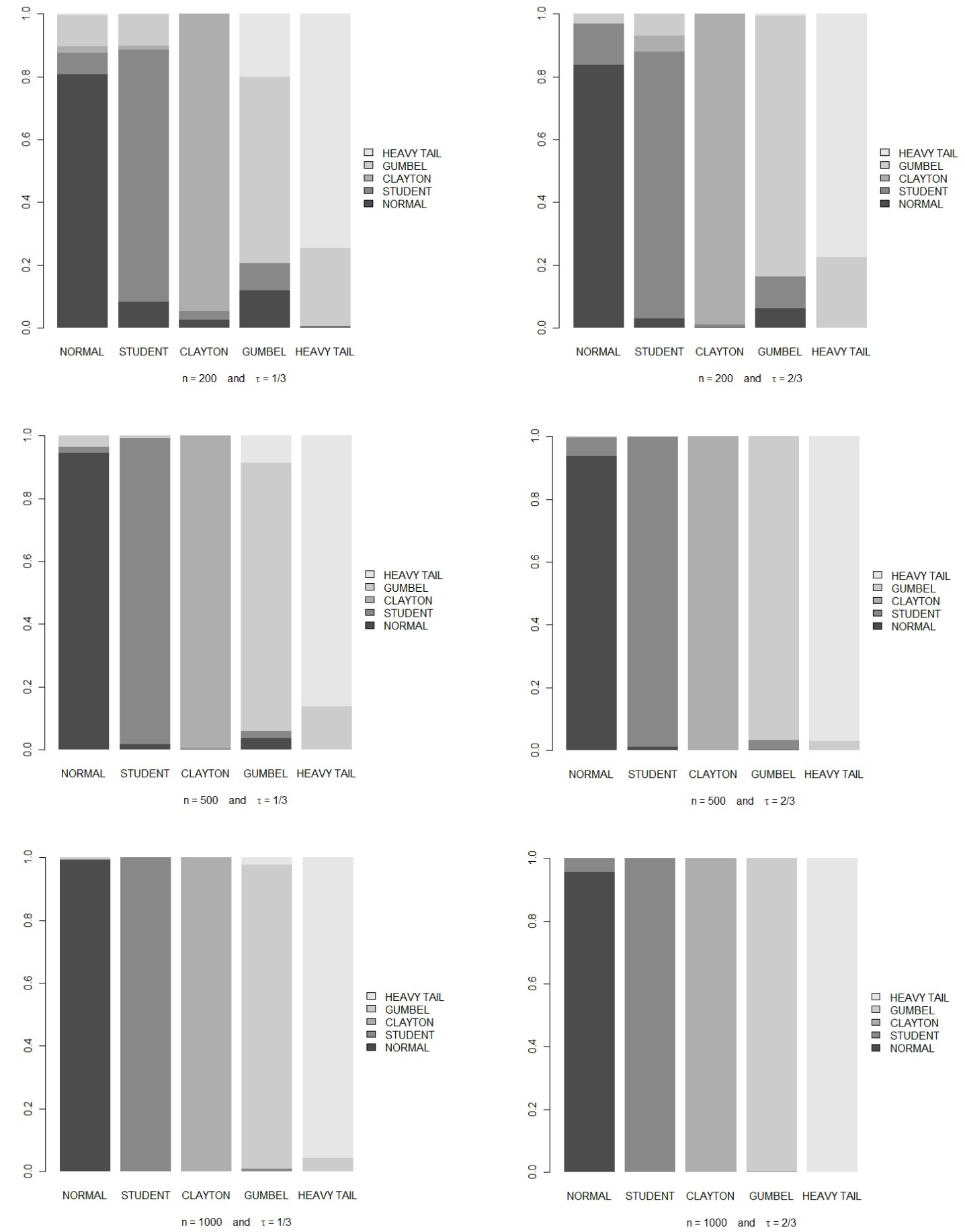

Figura 4.1: Gráficos de barras para proporção de seleção de cada modelo, segundo os critérios EAIC/EBIC, obtidos pelo método de estimação em dois passos para distribuições marginais com erros $\boldsymbol{S} \boldsymbol{S T}$. 

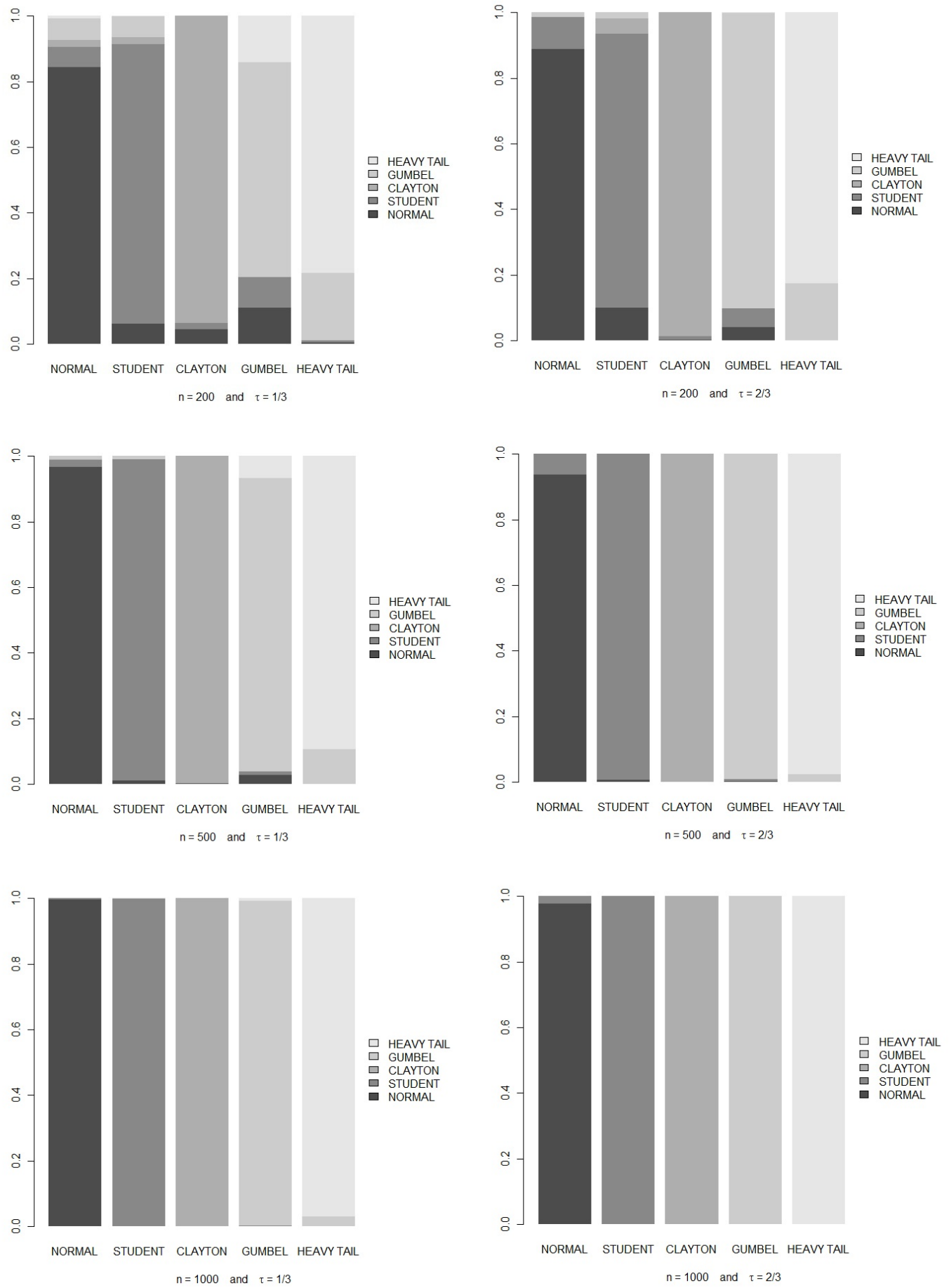

Figura 4.2: Gráficos de barras para proporção de seleção de cada modelo, segundo os critérios EAIC/EBIC, obtidos pelo método de estimação em dois passos para distribuições marginais com erros $\boldsymbol{S T}$. 

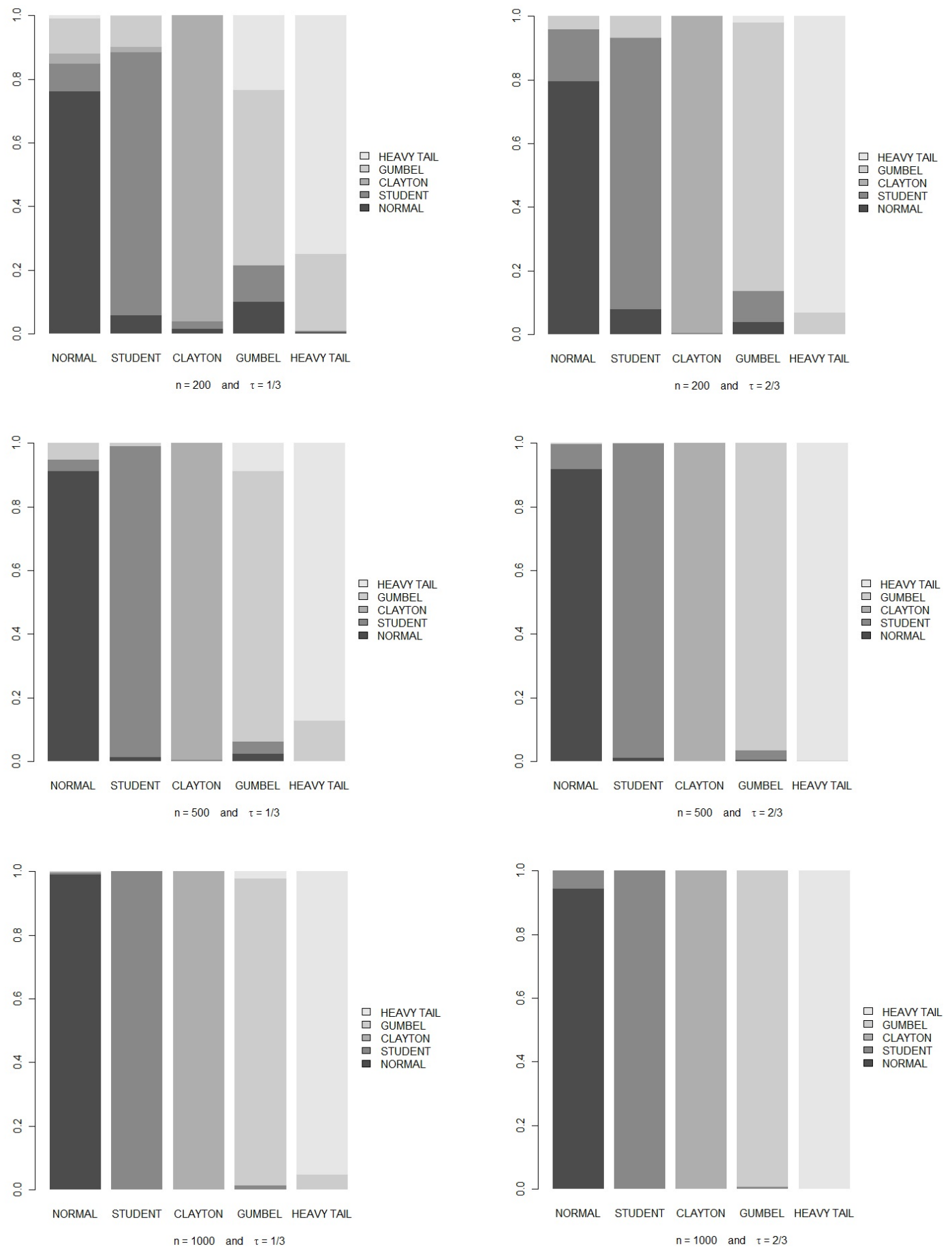

Figura 4.3: Gráficos de barras para proporção de seleção de cada modelo, segundo os critérios EAIC/EBIC, obtidos pelo método de estimação em um passo para distribuições marginais com erros $\boldsymbol{S} \boldsymbol{S T}$. 

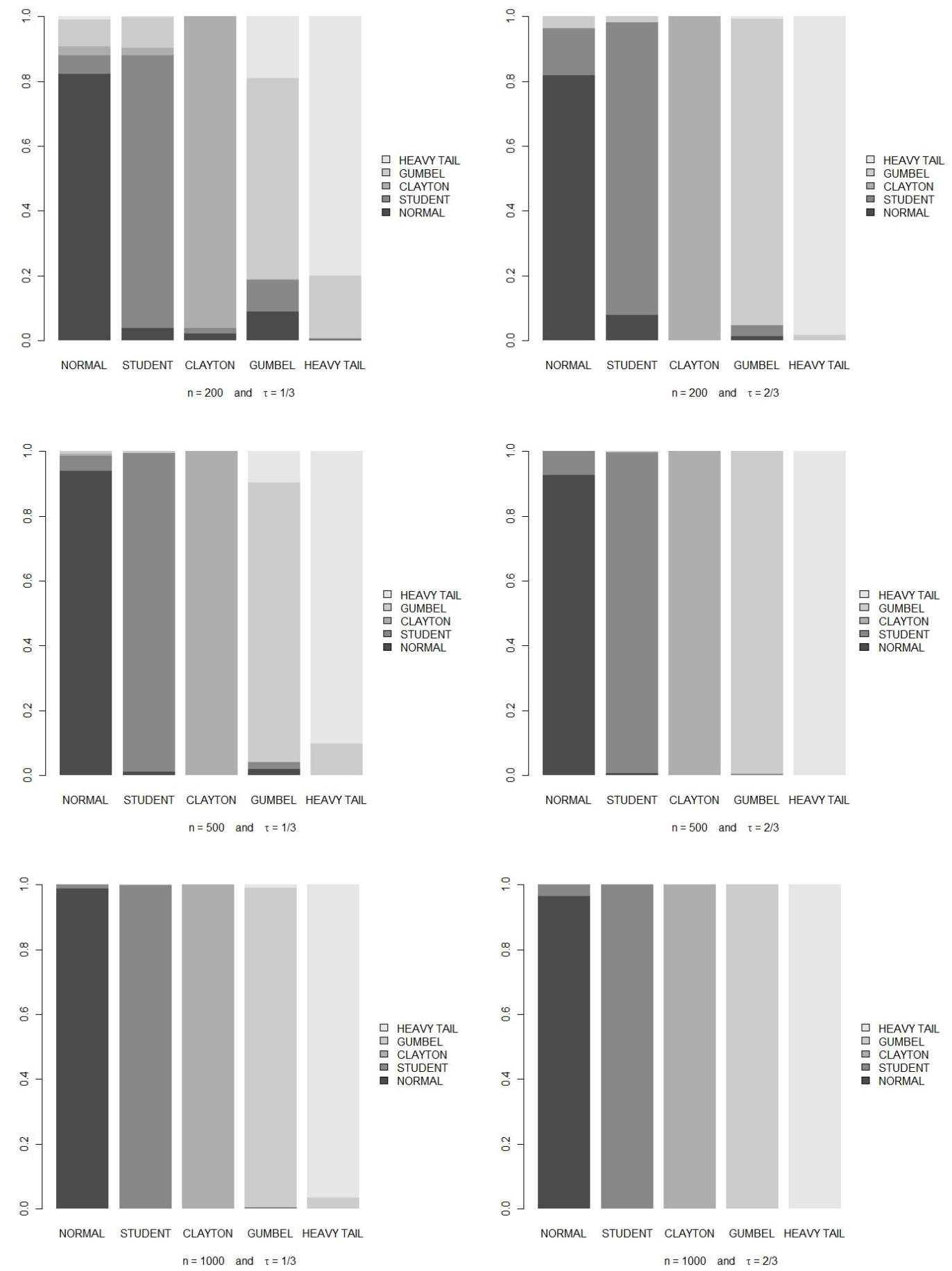

Figura 4.4: Gráficos de barras para proporção de seleção de cada modelo, segundo os critérios EAIC/EBIC, obtidos pelo método de estimação em um passo para distribuições marginais com erros $\boldsymbol{S T}$. 

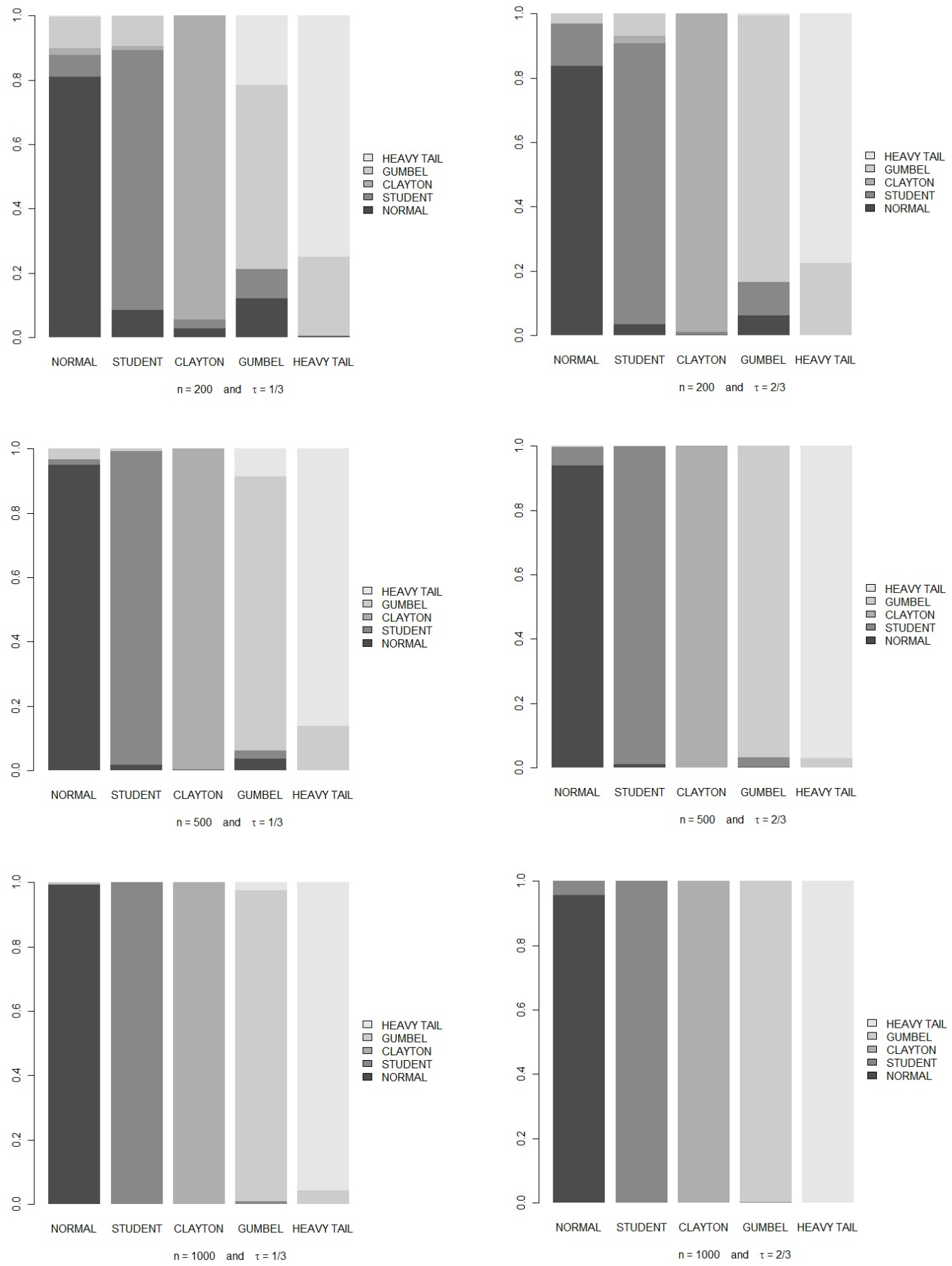

Figura 4.5: Gráficos de barras para proporção de seleção de cada modelo, segundo o critério DIC, obtidos pelo método de estimação em dois passos para distribuições marginais $\boldsymbol{S} \boldsymbol{S T}$. 

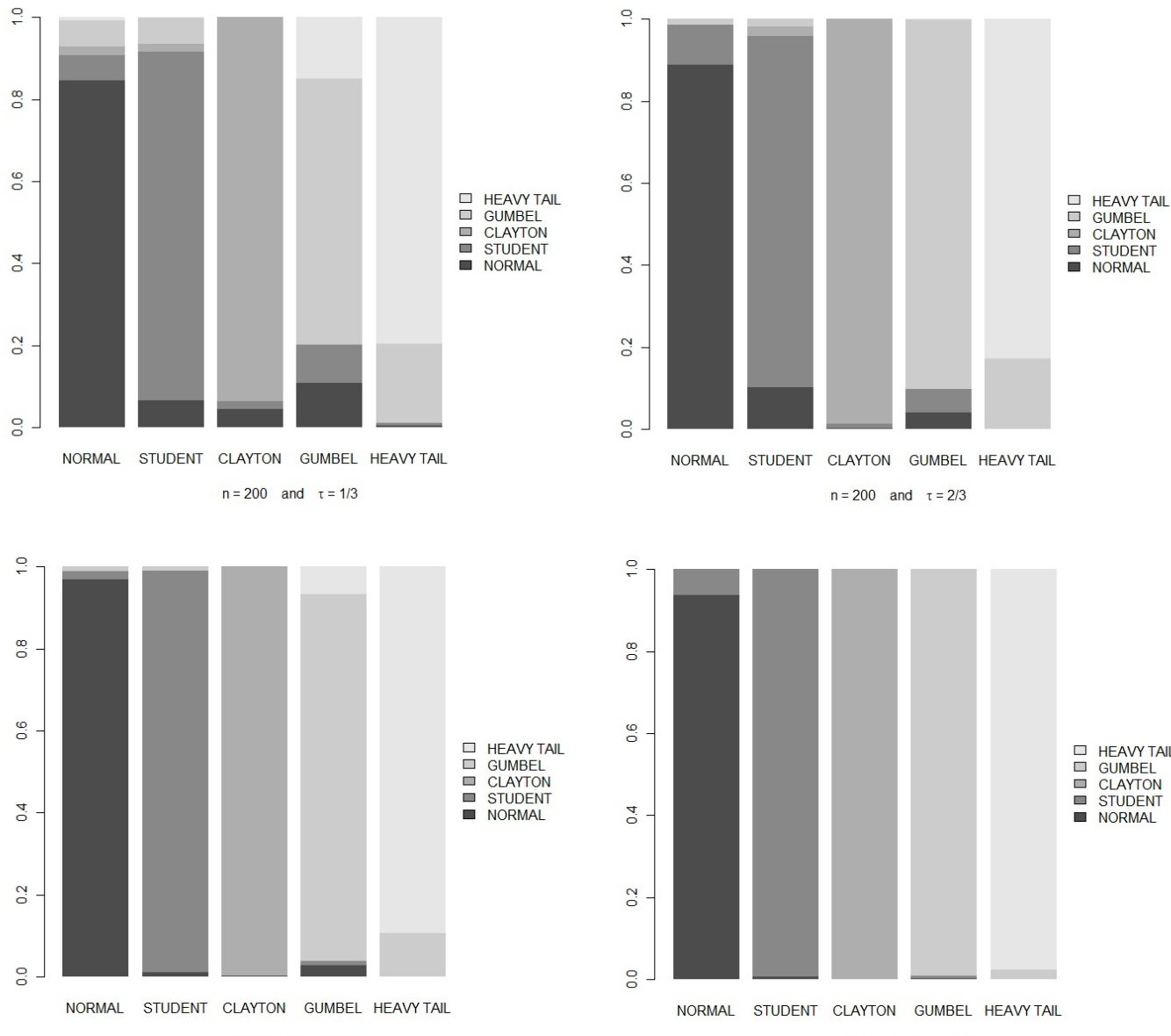

$n=500$ and $\tau=1 / 3$
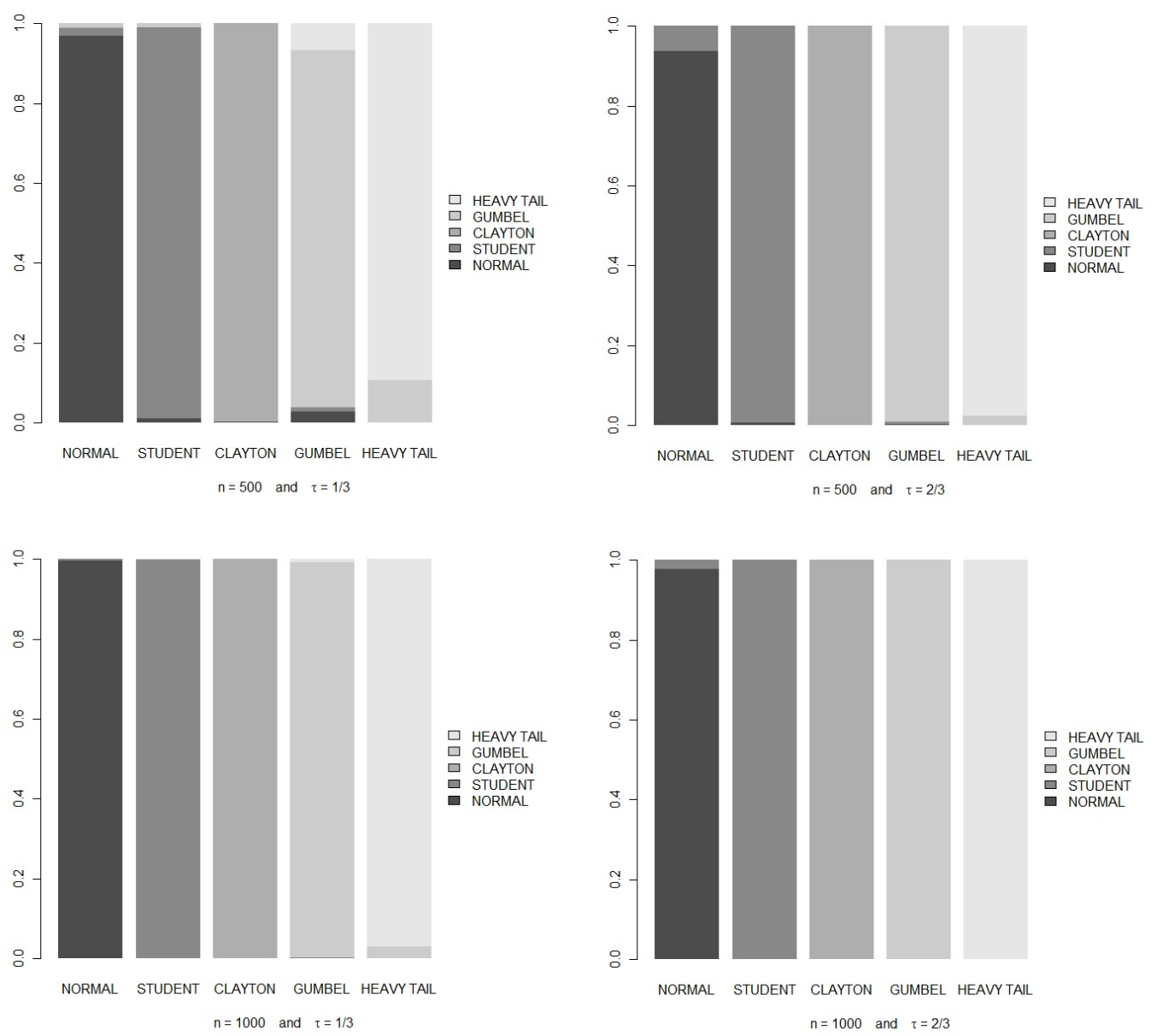

Figura 4.6: Gráficos de barras para proporção de seleção de cada modelo, segundo o critério DIC, obtidos pelo método de estimação em dois passos para distribuições marginais $\boldsymbol{S T}$. 

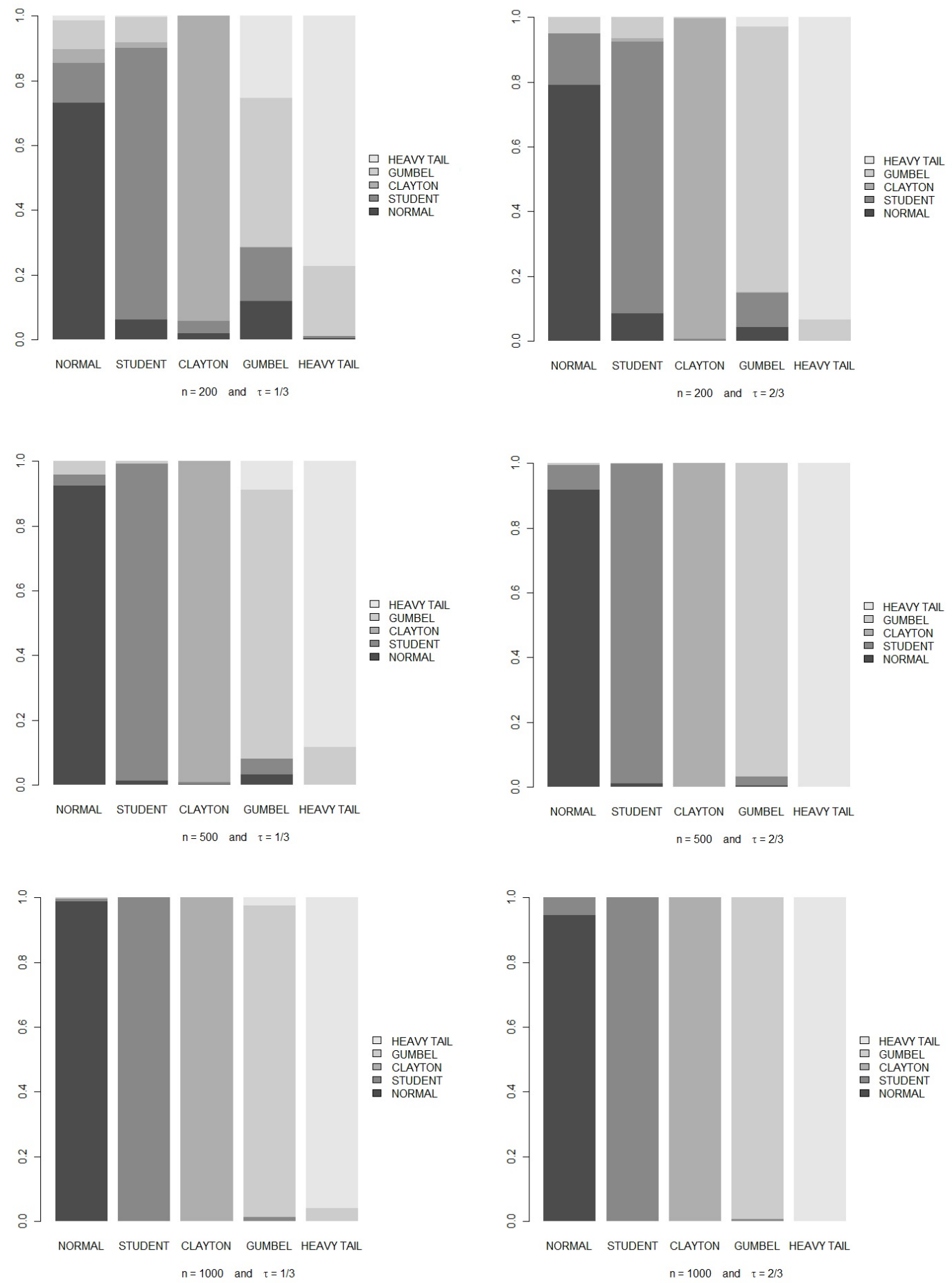

Figura 4.7: Gráficos de barras para proporção de seleção de cada modelo, segundo o critério DIC, obtidos pelo método de estimação em um passo para distribuições marginais $\boldsymbol{S S T}$. 

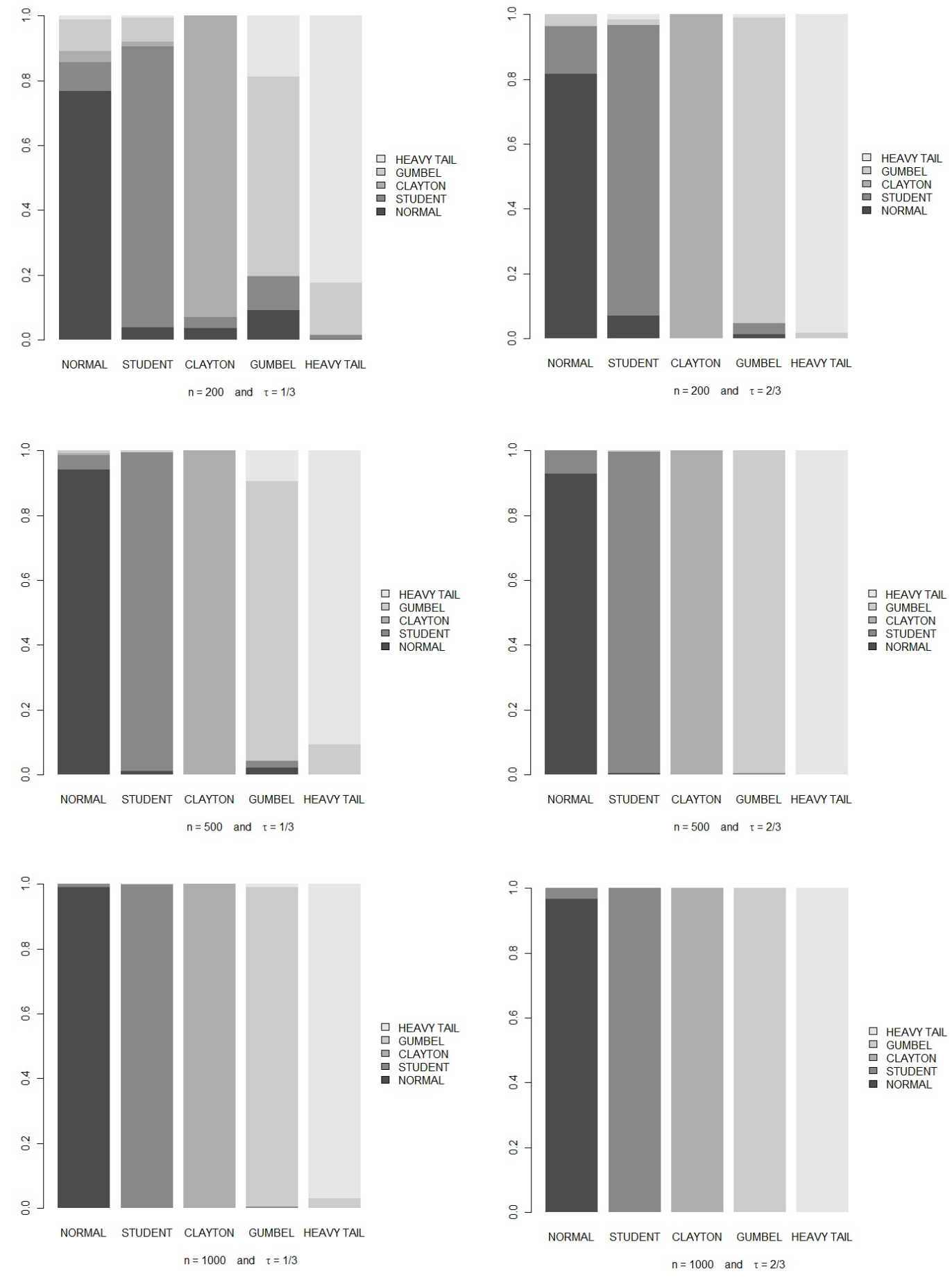

Figura 4.8: Gráficos de barras para proporção de seleção de cada modelo, segundo o critério DIC,obtidos pelo método de estimação em um passo para distribuições marginais $\boldsymbol{S T}$. 
Após a realização dos estudos de simulação, foi possível concluir que os resultados obtidos por meio da utilização do critério DIC (tabela 4.2 e gráficos 4.5 ao 4.8) foram similares aos obtidos pelos critérios EAIC/EBIC (tabela 4.1 e gráficos 4.1 ao 4.4), de maneira análoga aos resultados apresentados por Silva \& Lopes (2008).

Em acréscimo, os resultados sugerem que os dois métodos de estimação (em um passo e em dois passos) produzem resultados similares, no que se refere a seleção de modelos (tabelas 4.1, 4.2 e gráficos 4.1 ao 4.8). Porém, foi possível observar durante as simulações que, em alguns casos, há dependência paramétrica entre as distribuições marginais e esta dependência é levada em consideração somente quando os parâmetros são estimados em um passo. A figura 4.9 apresenta dois gráficos de dispersão para amostras das distribuições a posteriori dos parâmetros $\gamma_{x_{1}}$ e $\gamma_{x_{2}}$ quando estes foram estimados em um e dois passos. A amostra utilizada neste ajuste foi gerada a partir do modelo obtido pela cópula Clayton e distribuições marginais SST (um caso particular dos ajustes realizados no estudo de simulações). É possível notar que a estrutura de dependência entre os parâmetros é levada em consideração quando estes são estimados em um passo.

No que se refere a proporção de seleção do modelo de cópula correto, segundo o tamanho das séries, os resultados apresentados pelas tabelas 4.1, 4.2 mostram que para $\tau=1 / 3$, com $n=200$, as proporções de seleção do modelo correto foram superiores a 0,95 em apenas $10 \%$ dos casos estudados. Com $n=500$, em $45 \%$ dos casos foram observadas proporções superiores a 0,95 e com $n=1000$, em $100 \%$ dos casos foram observadas proporções superiores a 0,95. Já para $\tau=2 / 3$, com $n=200, n=500$ e $n=1000$, as porcentagens dos casos nos quais observou-se proporção de seleção do modelo correto superior a 0,95 foram, $25 \%, 80 \%$ e 95\%, respectivamente. Logo, no contexto deste trabalho, séries de tamanho $n=1000$ são suficientes para identificação do modelo de cópula correto.

Além disso, foi possível observar que o uso de baixos valores de dependência (dada 

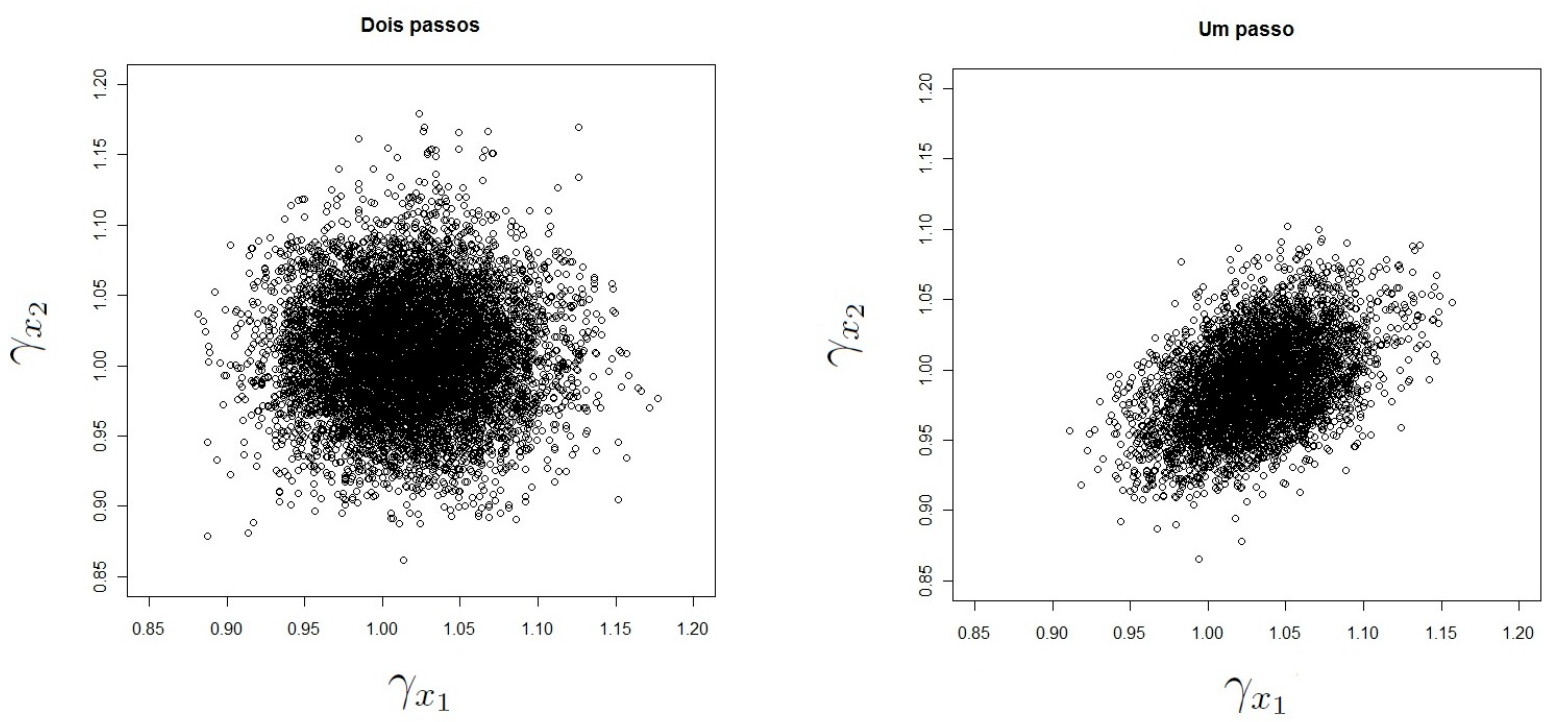

Figura 4.9: Gráfico de dispersão para amostras das distribuições a posteriori dos parâmetros $\gamma_{x_{1}}$ e $\gamma_{x_{2}}$, obtidas pela estimação em um e dois passos.

por $\tau$ ) e de funções de cópulas com características similares (como, por exemplo, da cópula t de Student para cópula normal é da cópula Gumbel para cópula Heavy Tail) resultaram em baixa proporção de seleção dos modelos corretos, principalmente para séries de tamanho reduzido (tabelas 4.1 e 4.2). Este fato foi previamente evidenciado por Silva \& Lopes (2008), em estudos de simulação com variáveis aleatórias marginais sem auto-correlação.

Outro fato observado foi o de que a proporção de seleção do modelo de cópula correto é, em alguns casos, maior para as modelos com distribuições marginais ST (tabelas 4.1 e 4.2). Um dos fatores que contribuiu para que isso ocorresse foi o de diferentes valores no parâmetro de assimetria proporcionarem melhor ajuste aos modelos que não foram utilizados para gerar a série, aumentando a similaridade entre estes modelos. A figura 4.10 apresenta um exemplo de melhor ajuste do modelo obtido pela cópula $C_{T}$ à dados com dependência assimétrica, quando os parâmetros de assimetria são estimados e não fixados iguais a um, ou seja, quando são utilizadas marginais $S S T$ e não $S T$. Para este exemplo, os modelos obtidos pela cópula $C_{T}$ (com distribuições marginais 
$S T$ e $S S T)$ e pela cópula $C_{C}$ (com distribuições marginais $S S T$ ) foram ajustados a um conjunto de dados gerado a partir da cópula $C_{C}$ com distribuições marginais $S S T$ e parâmetros de assimetria $\gamma_{x_{1}}=0.96$ e $\gamma_{x_{2}}=0.98$. Foi possível notar que as estimativas para estes parâmetros, no modelo que utilizou a cópula $C_{T}$, são bem superiores quando comparadas aos valores utilizados na geração. Porém, estas estimativas proporcionam um melhor ajuste aos dados, que tem estrutura de dependência assimétrica. Este fato foi comprovado pelos critérios de seleção.

No próximo capítulo são apresentadas aplicações dos modelos, obtidos pelas cópulas estáticas e variantes no tempo, à dados reais. 


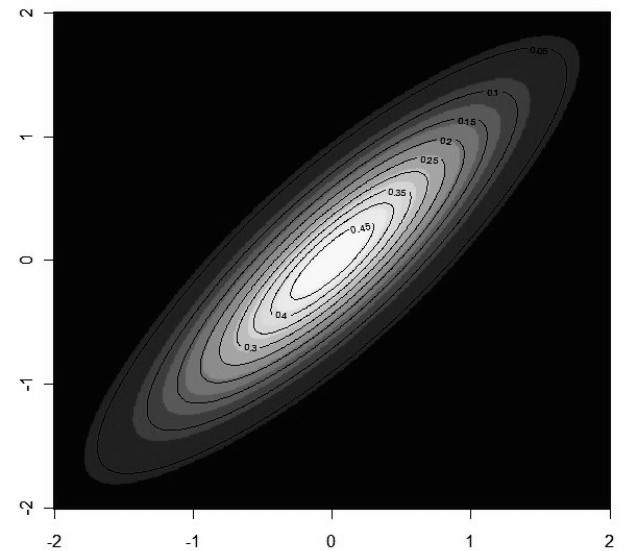

Cópula $C_{T}$,

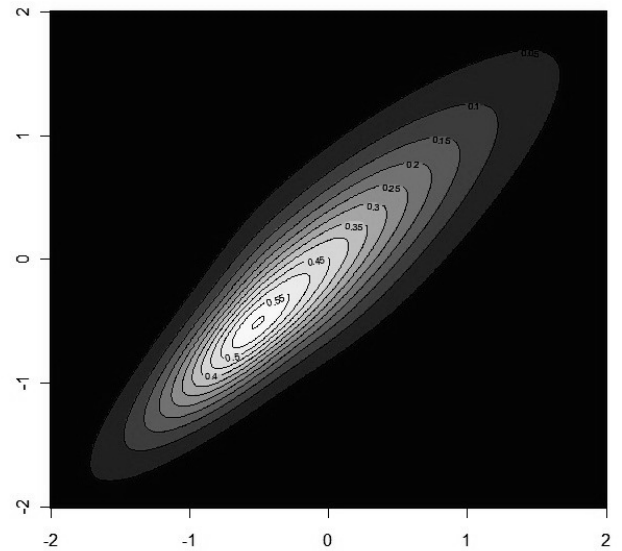

Cópula $C_{T}$,

$$
\gamma_{x_{1}}=1 \text { e } \gamma_{x_{2}}=1
$$

$$
\tilde{\gamma}_{x_{1}}=1.37 \text { e } \tilde{\gamma}_{x_{2}}=1.32
$$

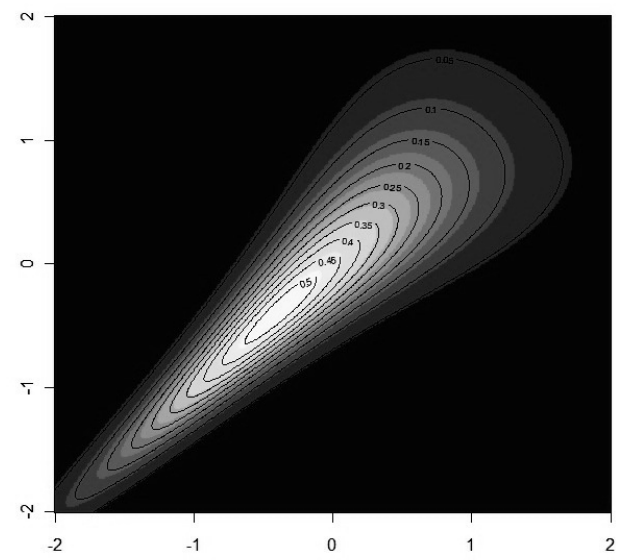

Cópula $C_{C}$,

$$
\tilde{\gamma}_{x_{1}}=1.03 \text { e } \quad \tilde{\gamma}_{x_{2}}=0.99
$$

Figura 4.10: Contorno de modelos bivariados com parâmetros de assimetria fixados (primeiro gráfico) e estimados (demais gráficos). 


\section{Capítulo 5}

\section{Aplicações}

Neste capítulo são apresentadas aplicações dos modelos utilizados no capítulo anterior e também de modelos nos quais a estrutura de dependência pode variar ao longo do tempo, de acordo com a teoria apresentada no capítulo 3.

Todas as estimações aqui apresentados foram efetuadas pelo método de estimação em um passo, para que a dependência paramétrica fosse levada em consideração. Os algoritmos MCMC utilizados foram implementados em linguagem $\mathrm{C}$ e executados no cluster Puma do Laboratório de Computação Científica Avançada - LCCA da USP.

\subsection{Especificação dos modelos}

Os modelos marginais utilizados neste capítulo, tanto para as distribuições cuja estrutura de dependência varia no tempo, quanto para as estáticas, foram os modelos AR(1)-GARCH(1,1) com erros SST. Este modelo foi especificados da seguinte 
maneira,

$$
\begin{aligned}
X_{i, t} & =\omega_{A R, x_{i}} X_{t-1}+\sqrt{h_{t}} \varepsilon_{x_{i}, t}, \\
h_{t} & =\omega_{x_{i}}+\alpha_{x_{i}} X_{t-i}^{2}+\beta_{x_{i}} h_{t-j}, \\
\varepsilon_{x_{i}, t} & \sim S S T\left(\nu_{x, i}, \gamma_{x, i}\right) .
\end{aligned}
$$

na qual, $\omega_{A R, x_{i}}$ é o parâmetro do modelo auto regressivo $A R$ na distribuição marginal $x_{i}$.

Para as estruturas de dependência, foram utilizadas as cinco cópulas apresentadas na seção 2.2 (Normal, t de Student, Clayton, Gumbel e Heavy Tail). Deste modo, foram obtidos dez modelos bivariados, considerando as cópulas estáticas e variantes no tempo. A permanência ou não dos parâmetros $\omega_{A R}$ e $\gamma$, em cada modelo, foi definida pelo critério $D I C$. As distribuições à priori utilizadas foram as mesmas do capítulo anterior, com exceção dos parâmetros referentes aos graus de liberdade que tiveram priori centrada em 5 , dos parâmetros $\omega_{A R_{1}}$ e $\omega_{A R_{2}}$ que tem distribuição $N^{T}(0,100)$ e dos parâmetros das cópulas variantes no tempo, $\omega_{C}, \beta_{C}$ e $\alpha_{C}$ que tem distribuição $N(0,100)$.

\section{$5.2 \quad \mathrm{O}$ conjunto de dados}

Os modelos obtidos foram ajustados à um conjunto de retornos diários de índices do mercado financeiro. Estes índices foram, o Merval (MERV) da Argentina, o Standard 8 Poor's 500 (SP500) dos E.U.A., o Ibovespa (BVSP) do Brasil e o Indice de Precios y Cotizaciones (ICP) do México, para o período de 03/01/2005 à 29/12/2011, totalizando 1606 observações para os retornos. Os dados foram obtidos da fonte http://finance.yahoo.com e foram consideradas válidas somente as observações nas quais os valores dos quatro índices puderam ser observados. A tabela 5.1 exibe algumas estatísticas descritivas e as figuras 5.1 e 5.2 exibem as séries dos índices e dos retornos dos índices, respectivamente. 
Tabela 5.1: Estatísticas descritivas dos índices utilizados.

\begin{tabular}{lrrrr}
\hline & MERV & ICP & BVSP & SP500 \\
\hline Média & $7.30 \mathrm{e}-05$ & $6.15 \mathrm{e}-06$ & $9.85 \mathrm{e}-05$ & $1.30 \mathrm{e}-04$ \\
Variância & $1.60 \mathrm{e}-05$ & $8.86 \mathrm{e}-06$ & $1.63 \mathrm{e}-05$ & $9.58 \mathrm{e}-06$ \\
Mínimo & -0.02590 & -0.01893 & -0.02419 & -0.01453 \\
Máximo & 0.02086 & 0.02084 & 0.03094 & 0.02222 \\
Curtose & 8.42579 & 11.2802 & 9.00980 & 7.95734 \\
Assimetria & -0.6610 & -0.2663 & 0.05262 & 0.22803 \\
& & & &
\end{tabular}
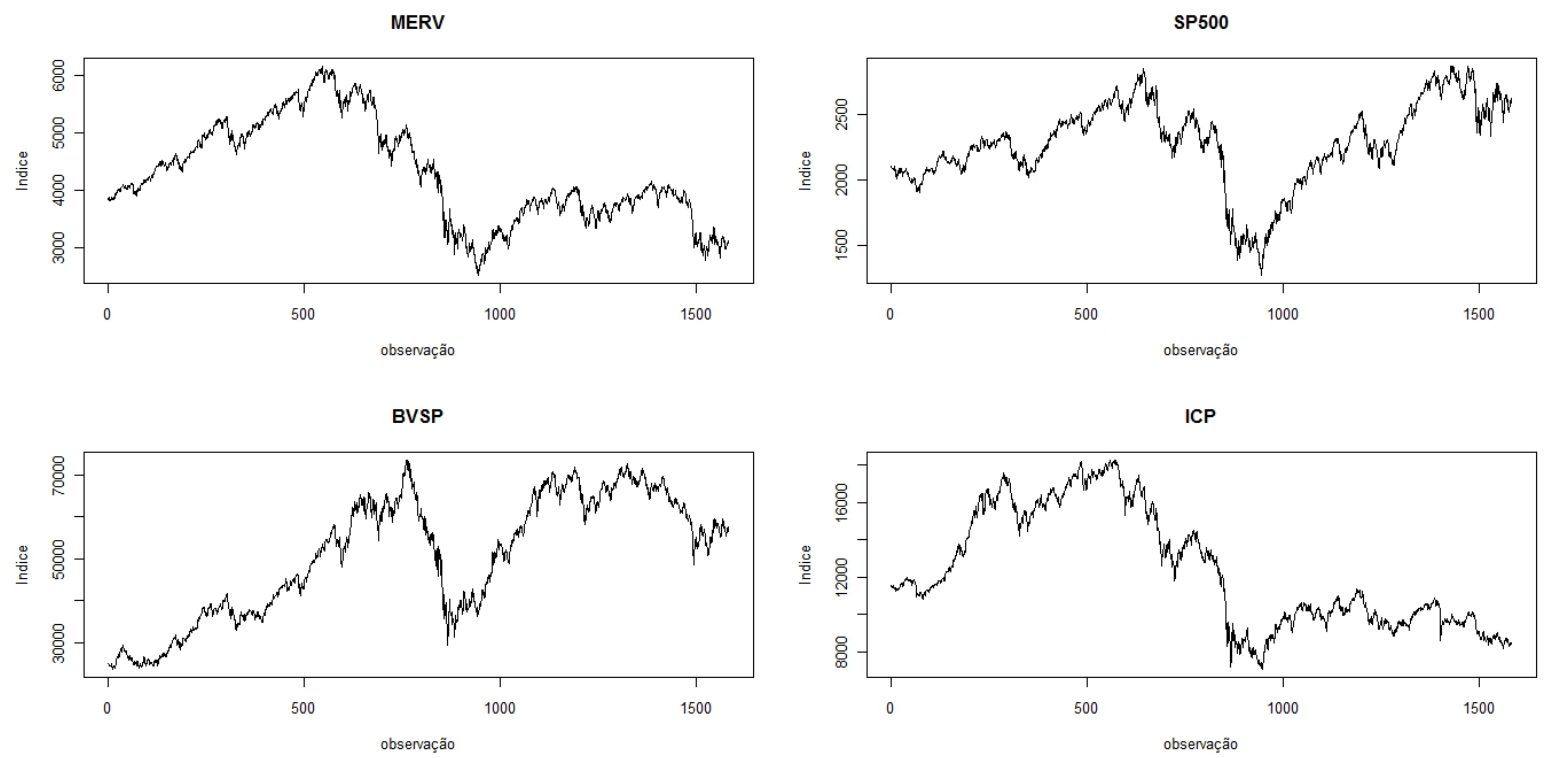

Figura 5.1: Gráfico das séries para os quatro índices utilizados, MERV, SP500, BVSP e ICP, para o período de 03/01/2005 à 29/12/2011.

\subsection{Resultados}

Foram realizados ajustes à séries bivariadas, obtidas a partir das seis possíveis combinações entre os índices. Estas foram ICP X BVSP, MERV X ICP, MERV X BVSP, MERV X SP500, SP500 X BVSP e SP500 X ICP.

Antes de ajustar os modelos com estrutura de dependência variante, foi preciso 

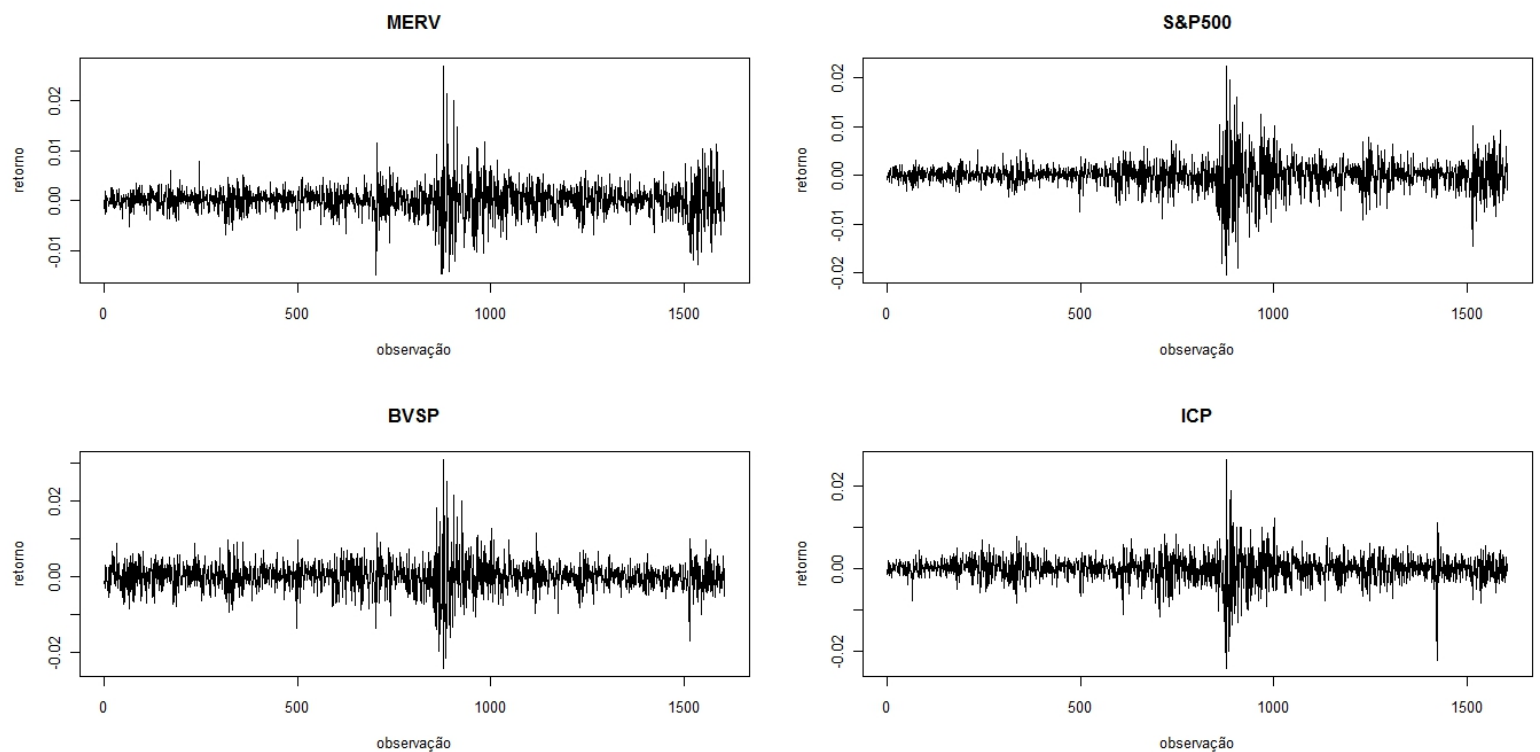

Figura 5.2: Gráfico das séries de retornos dos quatro índices utilizados, MERV, SP500, BVSP e ICP, para o período de 03/01/2005 à 29/12/2011, totalizando 1606 observações.

mostrar que são válidas as igualdades 3.2 e 3.3. Para tal, foram feitos ajustes de modelos de regressão considerando os resíduos da primeira distribuição marginal, contra a primeira defasagem dos retornos e contra a primeira defasagem do quadrado dos retornos da segunda distribuição marginal. Como os resíduos dos modelos marginais ajustados tem distribuição $S S T$, foram utilizados modelos de regressão para variável resposta com distribuição t de Student assimétrica. Estas regressões foram realizadas com a utilização do pacote sn (Azzalini, 2011) e os resultados dos testes são exibidos na tabela 5.2.

A aleatoriedade dos resíduos foi verificada pelos testes de Ljung-Box e a convergência dos algoritmos MCMC foi monitorada por meio de gráficos e dos testes de Heidelberger e Welch, disponíveis no pacote CODA (Plummer et al., 2006) do software R Development Core Team (2010).

As tabelas 5.3 à 5.8 apresentam as estimativas dos parâmetros, dos desvios padrões e os valores do critério DIC, que foi utilizado na seleção dos modelos. Os resultados obtidos pelos critérios EAIC e EBIC foram omitidos, pois corroboram com os do DIC. 
Tabela 5.2: Valor p obtido pelos testes de significância do coeficiente dos retornos e dos quadrados dos retornos nos modelos de regressão. Estas regressões foram realizadas para mostrar que são válidas as equações 3.2 e 3.3. As linhas representam a primeira defasagem dos erros utilizada nos testes e as colunas representam os retornos e quadrados dos retornos.

\begin{tabular}{|c|c|c|c|c|}
\hline & $r t_{[t-1]}$ MERV & $r t_{[t-1]} \mathbf{S P 5 0 0}$ & $r t_{[t-1]} \mathbf{B V S P}$ & $r t_{[t-1]}$ IPC \\
\hline$\varepsilon_{\mathbf{t}}$ MERV & - & 0.1201987 & 0.2916478 & 0.2752796 \\
\hline$\varepsilon_{\mathrm{t}} \mathrm{SP} 500$ & 0.06232049 & - & 0.3976578 & 0.6496909 \\
\hline$\varepsilon_{\mathrm{t}} \mathrm{BVSP}$ & 0.28634151 & 0.6996082 & - & 0.7660801 \\
\hline \multirow[t]{2}{*}{$\varepsilon_{\mathrm{t}} \mathrm{IPC}$} & 0.20543118 & 0.9094270 & 0.8884487 & - \\
\hline & $r t_{[t-1]}^{2}$ MERV & $r t_{[t-1]}^{2} \mathbf{S P 5 0 0}$ & $r t_{[t-1]}^{2} \mathbf{B V S P}$ & $r t_{[t-1]}^{2} \mathbf{I P C}$ \\
\hline$\varepsilon_{\mathbf{t}}$ MERV & _ & 0.4143792 & 0.61898569 & 0.31699856 \\
\hline$\varepsilon_{\mathrm{t}} \mathrm{SP} 500$ & 0.9034765 & - & 0.33198695 & 0.08699984 \\
\hline$\varepsilon_{\mathbf{t}} \mathrm{BVSP}$ & 0.2518343 & 0.7593142 & - & 0.45338741 \\
\hline$\varepsilon_{\mathrm{t}} \mathrm{IPC}$ & 0.1186658 & 0.2920894 & 0.09681698 & - \\
\hline
\end{tabular}

Os gráficos 5.3 à 5.7, mostram a variação dos parâmetros $\rho_{N} \mathrm{e} \rho_{T}$, para as cópulas Normal e Student, além das variações nas dependências de cauda inferior $\left(\tau_{C}\right)$ para cópula Clayton e de cauda superior $\left(\tau_{G}\right.$ e $\left.\tau_{H}\right)$ para as cópulas Gumbel e Heavy Tail. Nas tabelas 5.3 a 5.8, a sigla (V.T.) indica a versão variante no tempo da cópula e nos gráficos 5.3 a 5.8, as linhas horizontais contínuas representam a estimativa pontual para os parâmetros de cópula nos modelos estáticos e as linhas horizontais tracejadas seus intervalos de credibilidade. 


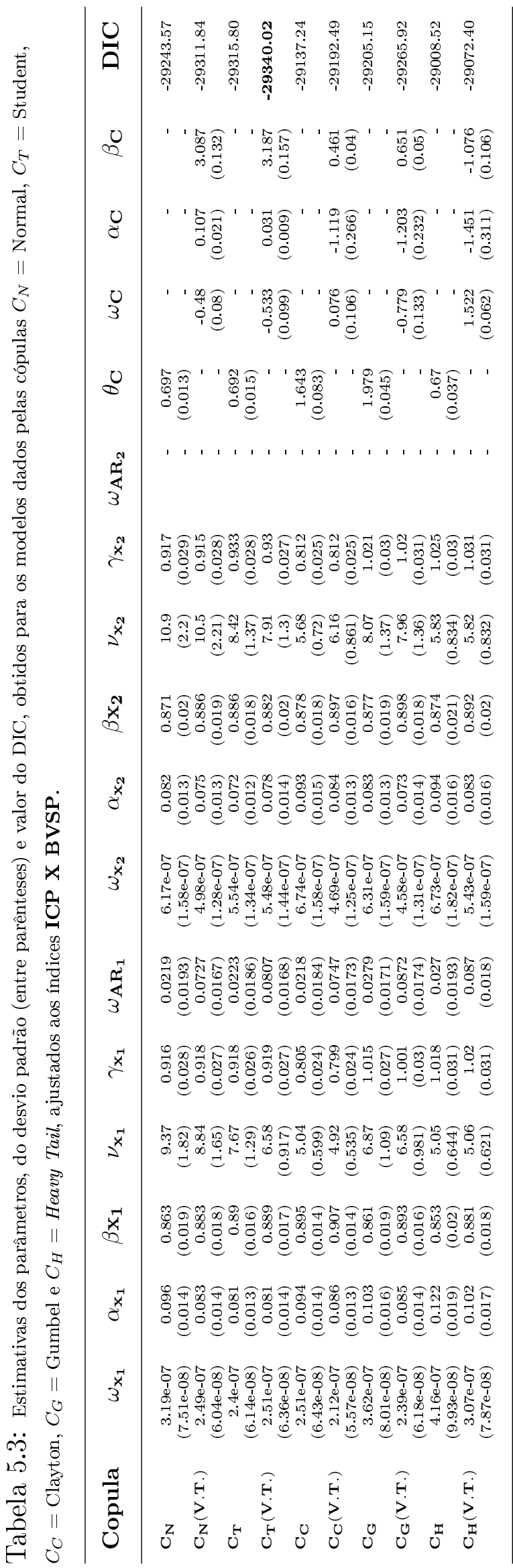

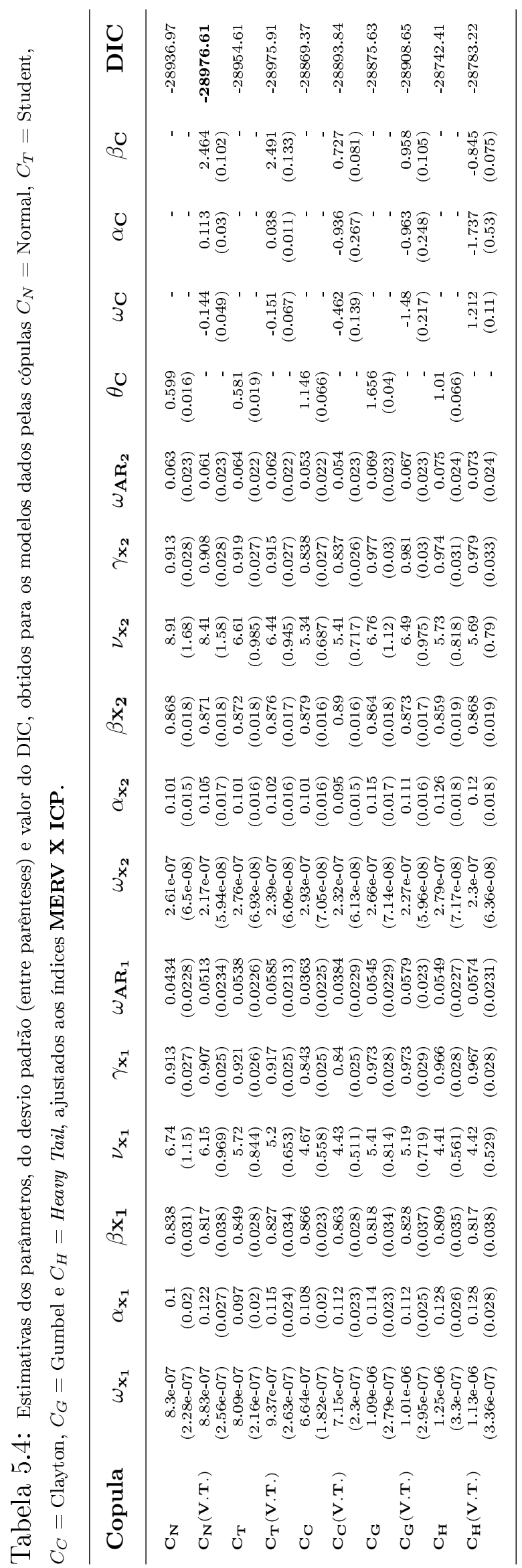




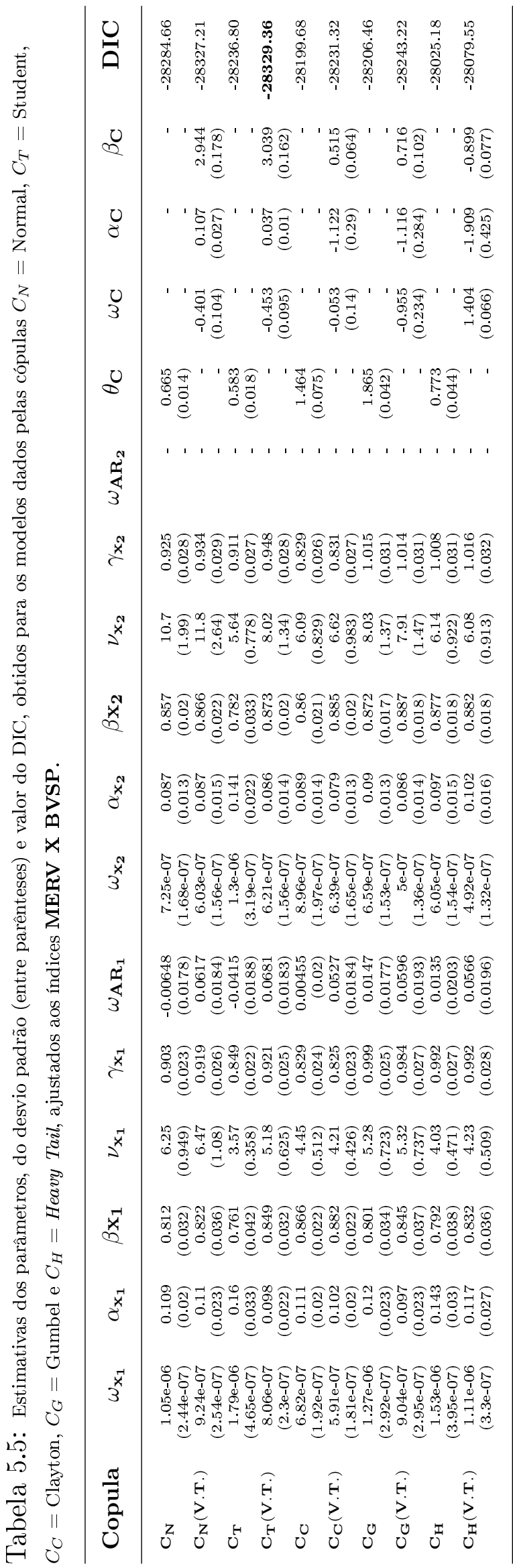

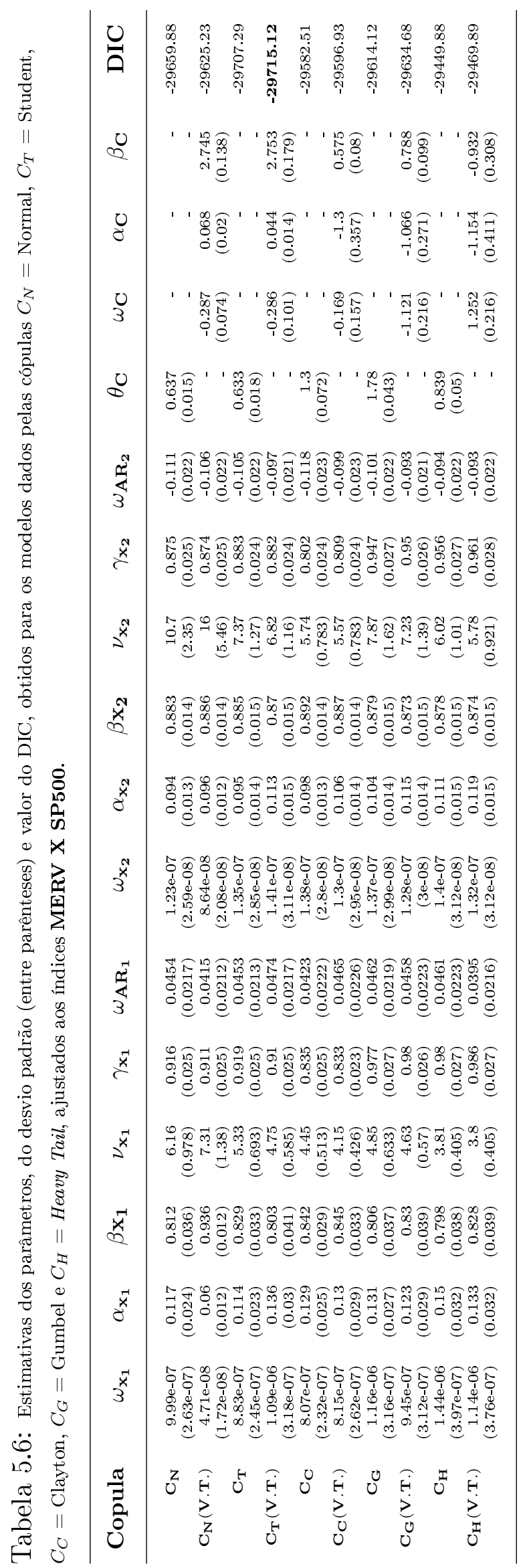




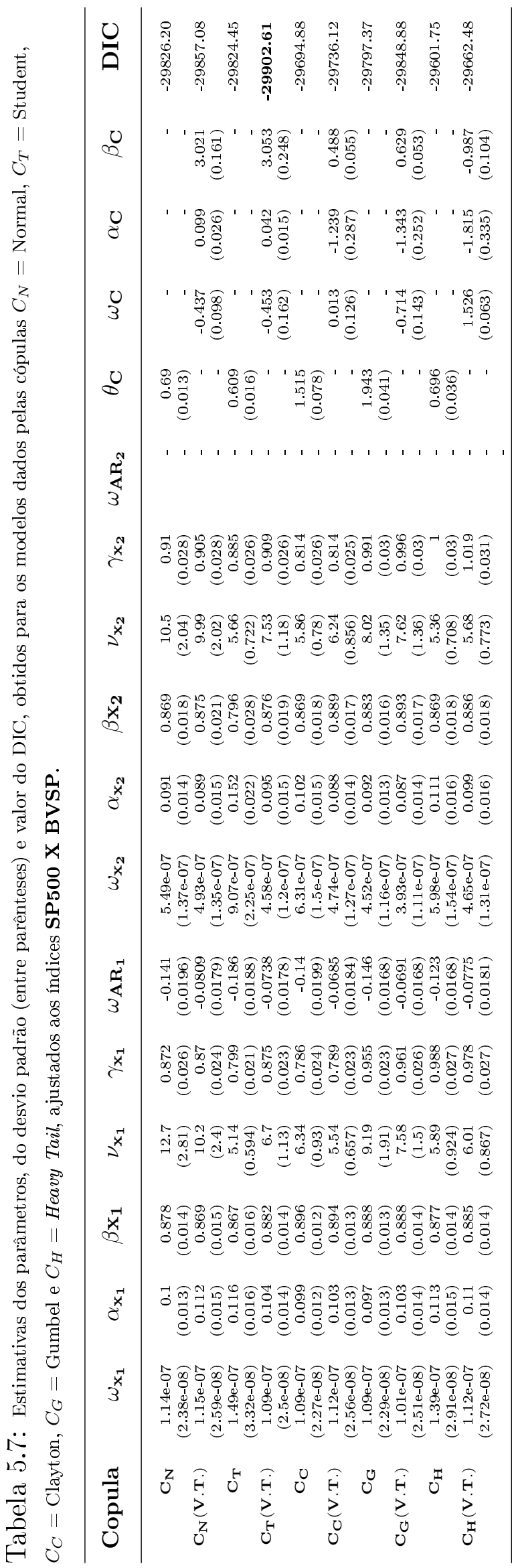

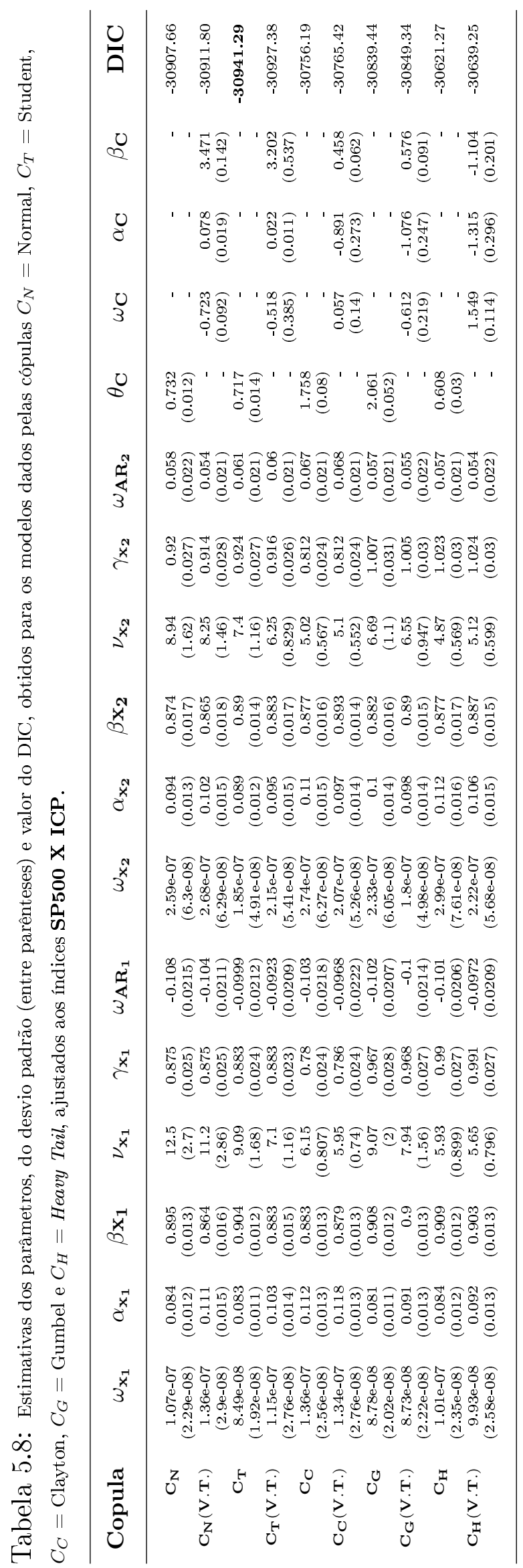



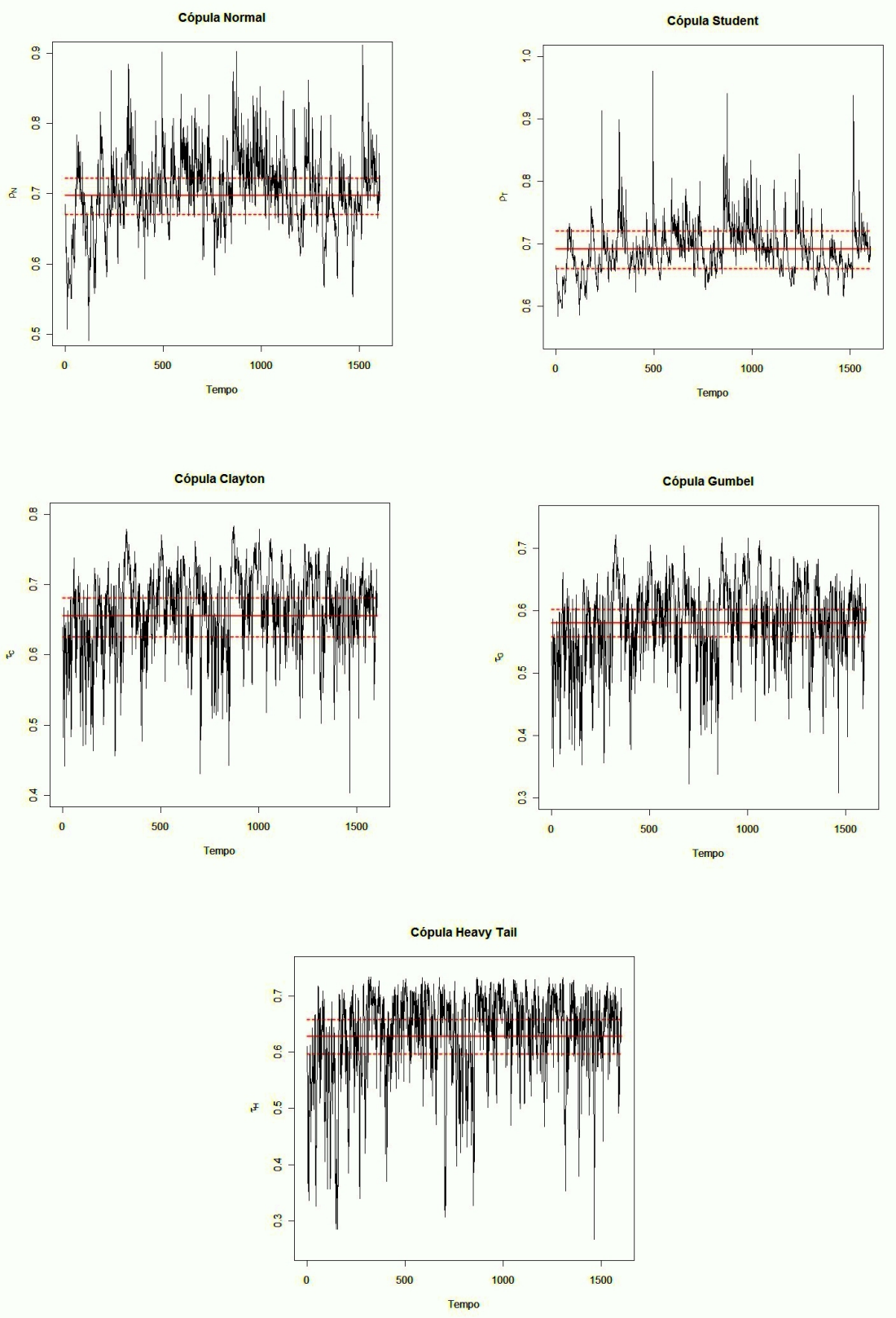

Figura 5.3: ICP X BVSP - variações no tempo dos coeficientes de correlação para as cópulas elípticas e variações no tempo das medidas de dependência nas caudas para as cópulas Clayton, Gumbel eHeavy Tail. 

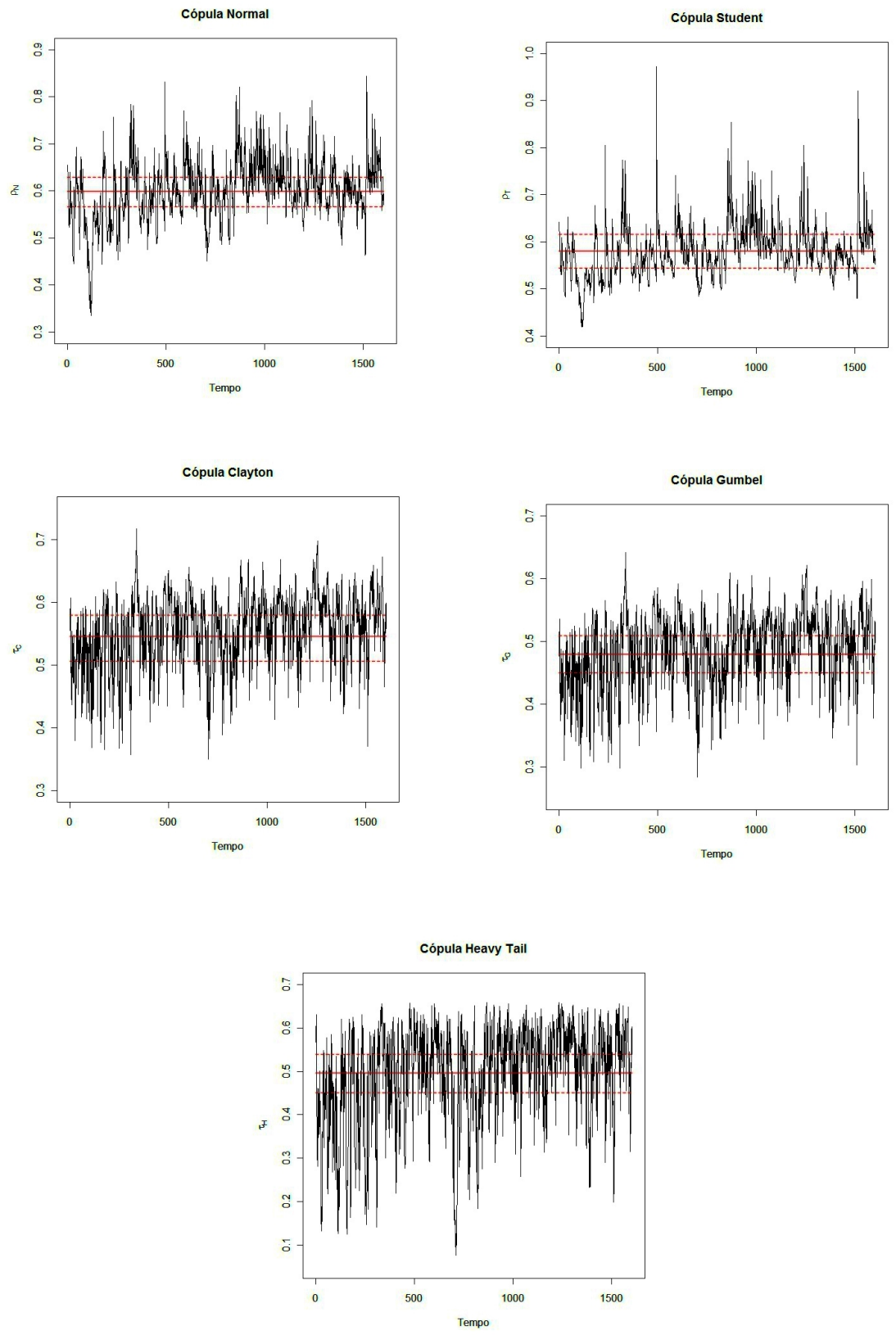

Figura 5.4: MERV X ICP - variações no tempo dos coeficientes de correlação para as cópulas elípticas e variações no tempo das medidas de dependência nas caudas para as cópulas Clayton, Gumbel eHeavy Tail. 

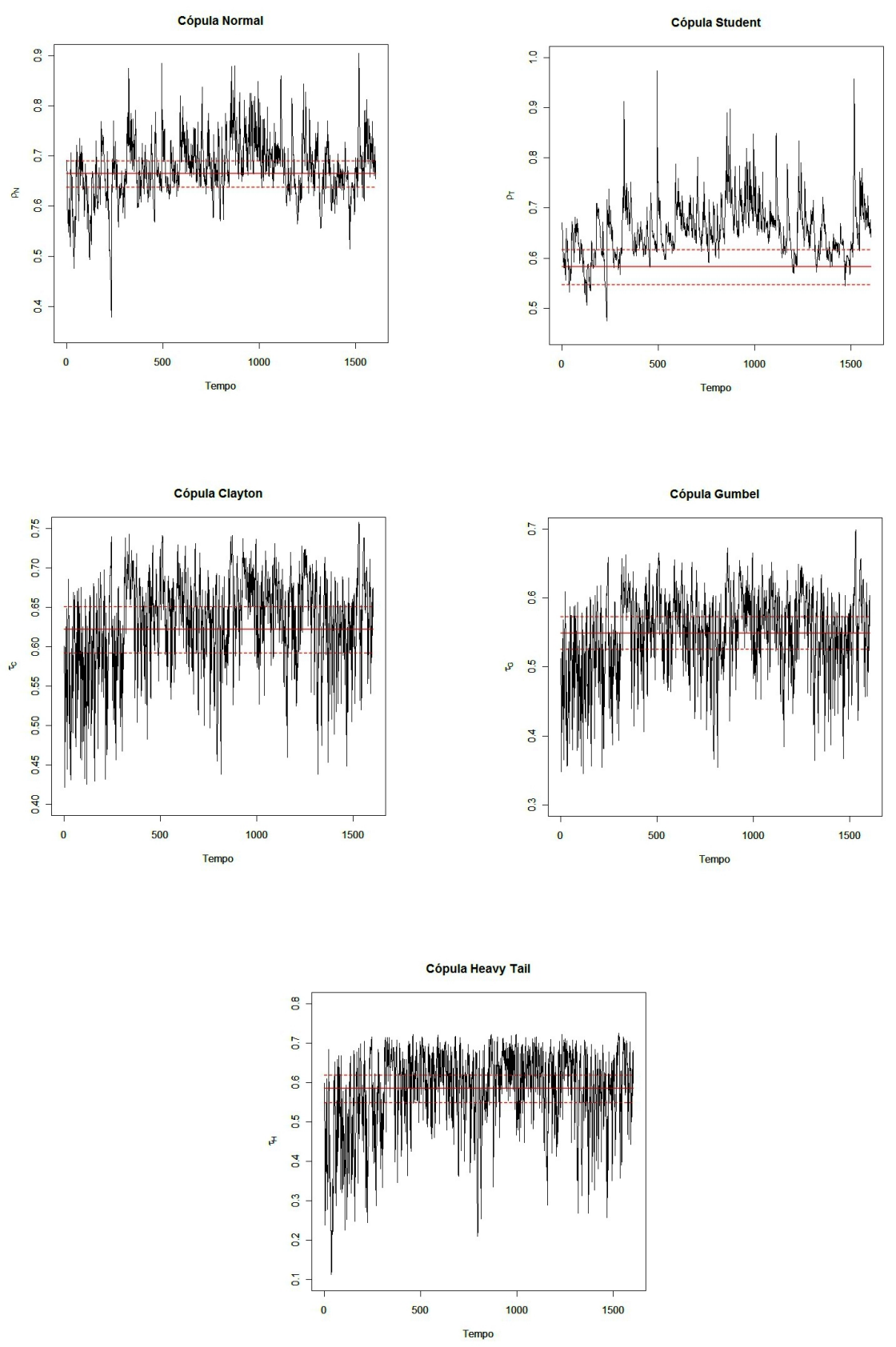

Figura 5.5: MERV X BVSP - variações no tempo dos coeficientes de correlação para as cópulas elípticas e variações no tempo das medidas de dependência nas caudas para as cópulas Clayton, Gumbel eHeavy Tail. 

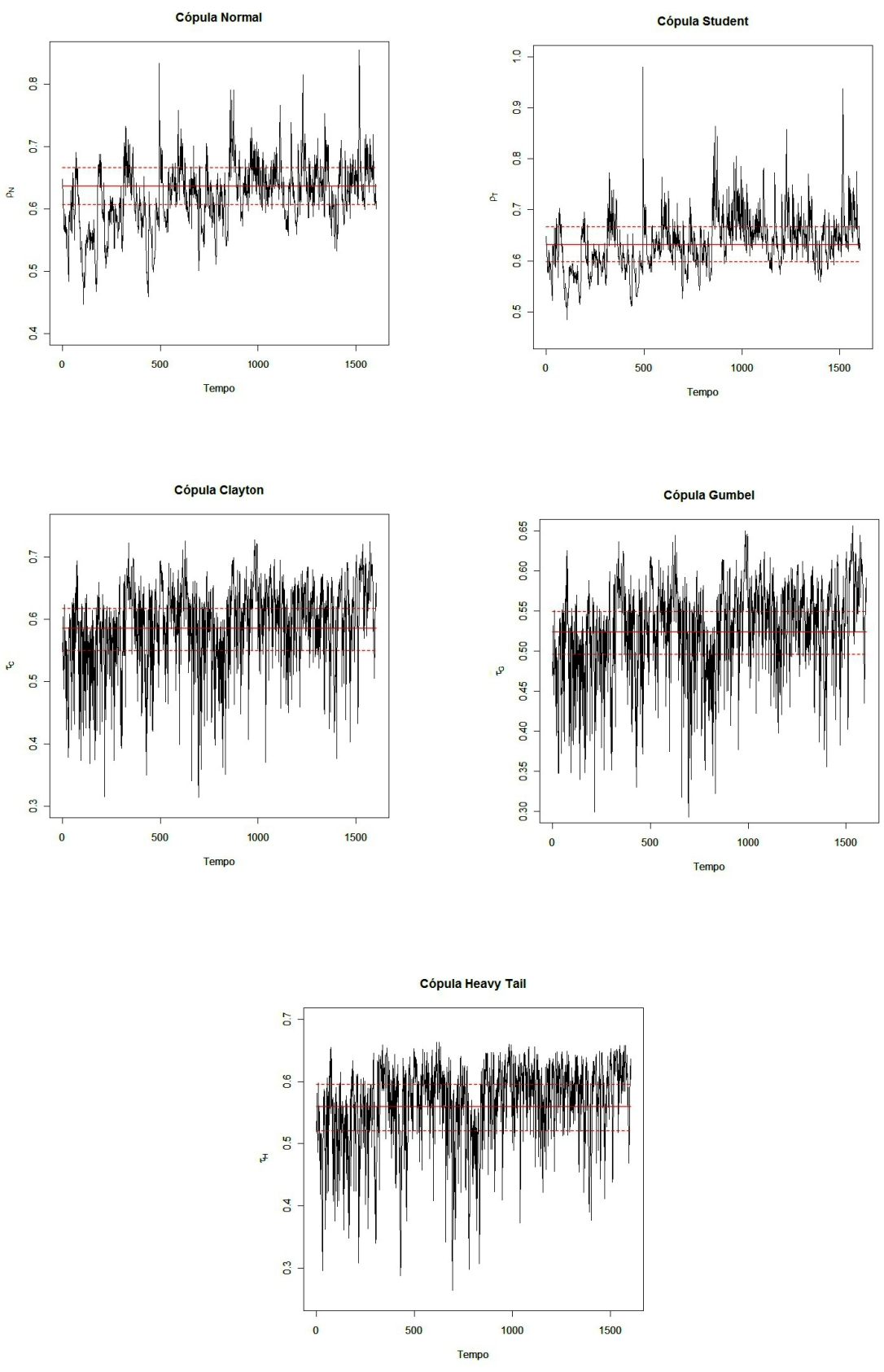

Figura 5.6: MERV X SP500 - variações no tempo dos coeficientes de correlação para as cópulas elípticas e variações no tempo das medidas de dependência nas caudas para as cópulas Clayton, Gumbel eHeavy Tail. 

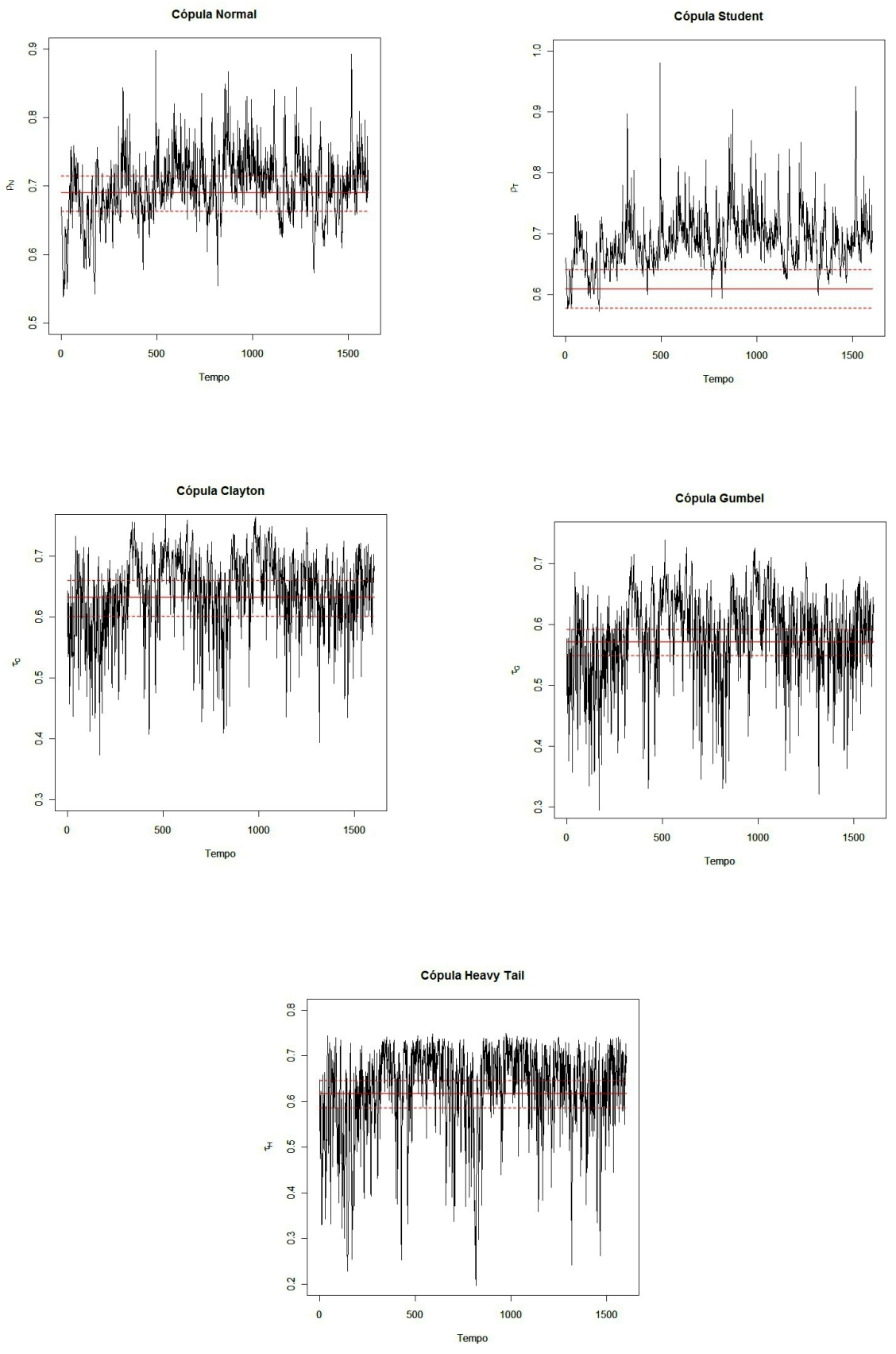

Figura 5.7: SP500 X BVSP - variações no tempo dos coeficientes de correlação para as cópulas elípticas e variações no tempo das medidas de dependência nas caudas para as cópulas Clayton, Gumbel eHeavy Tail. 

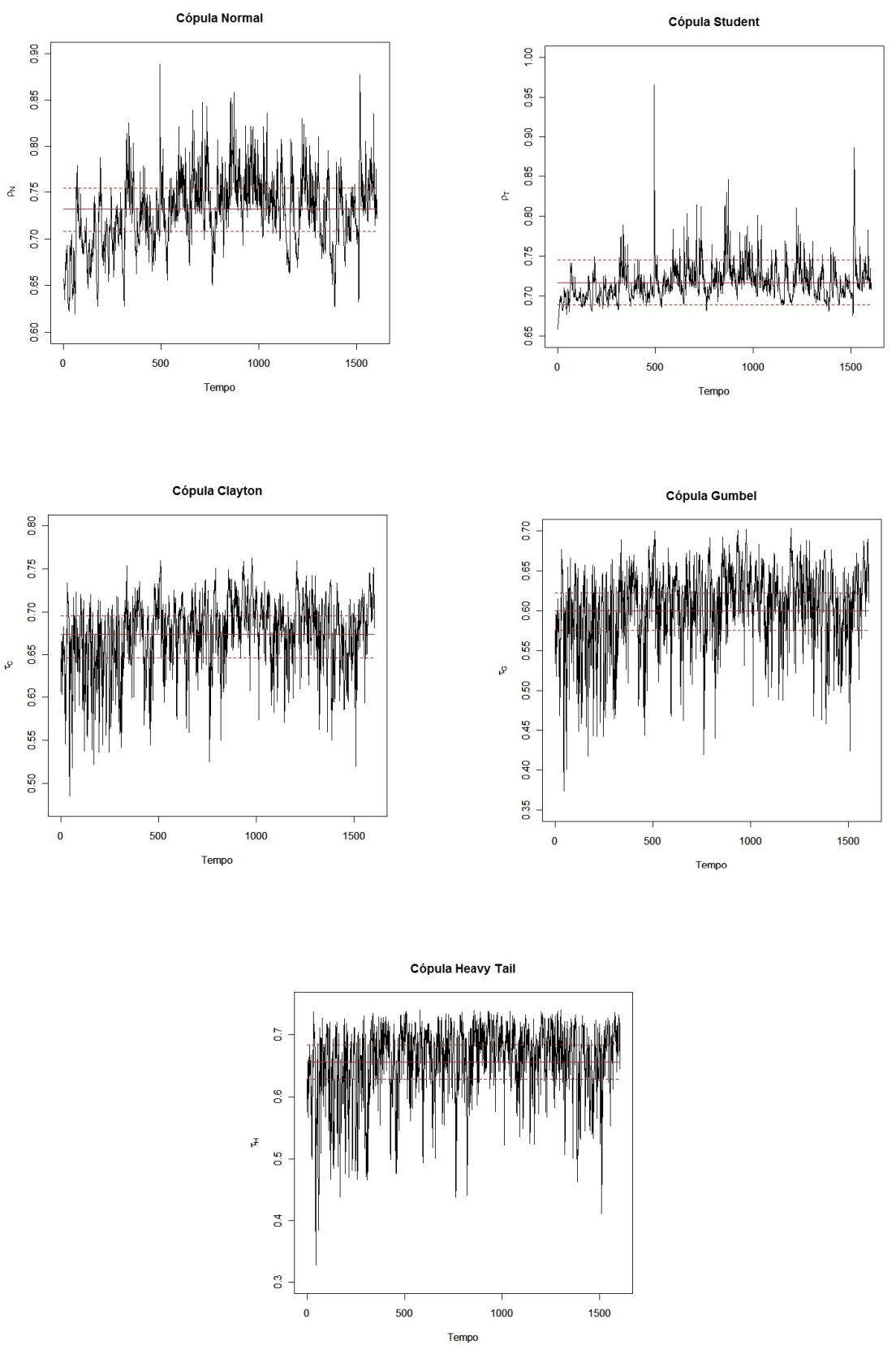

Figura 5.8: SP500 X ICP - variações no tempo dos coeficientes de correlação para as cópulas elípticas e variações no tempo das medidas de dependência nas caudas para as cópulas Clayton, Gumbel eHeavy Tail. 


\subsection{Conclusões e Considerações Finais}

De todos os modelos com estrutura de dependência variante no tempo ajustados, 96,3\% tiveram melhor ajuste do que sua versão estática. Patton (2006a) e Abbara (2009) também constataram melhor ajuste dos modelos variantes no tempo. Em acréscimo, quatro dos seis pares de séries (ICP X BVSP, MERV X BVSP, MERV X SP500 e SP500 X BVSP) tiveram como melhor ajuste o obtido pelo modelo gerado a partir da cópula t de Student variante no tempo. As duas restantes, MERV X ICP e SP500 X ICP, tiveram melhor ajuste pelos modelos obtidos pela cópula Normal variante no tempo e t de Student estática, respectivamente. Também foi observado que a dependência entre os retornos, assim como a volatilidade, é maior nos períodos de queda nos índices (figura 5.9). Este fato foi previamente observado nos trabalhos supracitados, realizados no mesmo contexto.

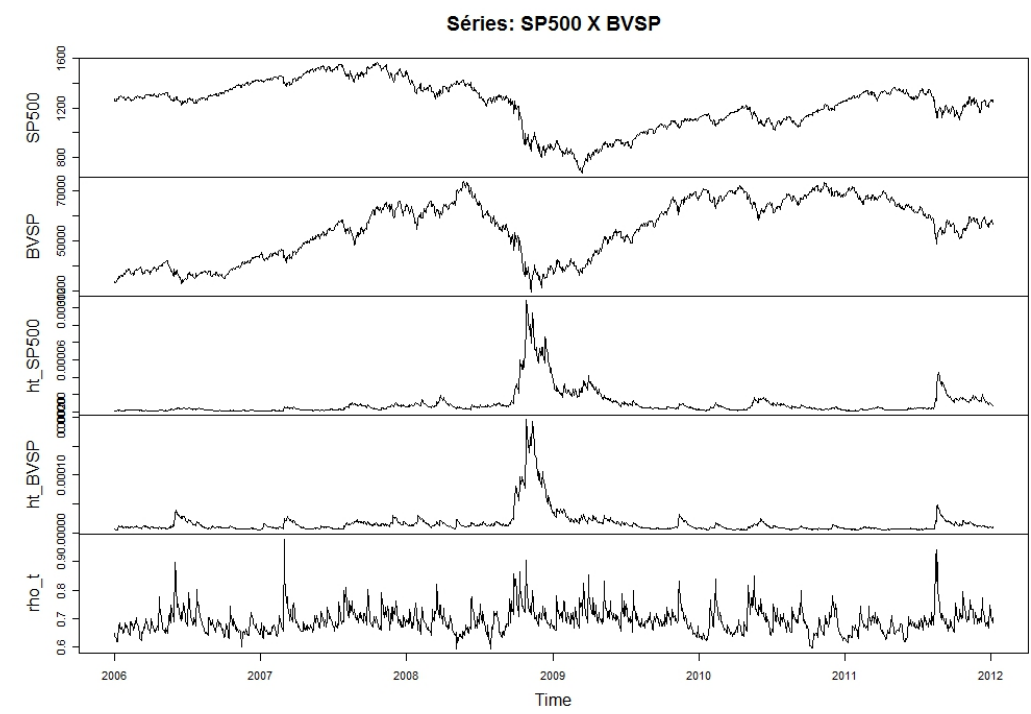

Figura 5.9: Séries dos índices SP500 e BVSP, volatilidade estimada para SP500 e BVSP (rt SP500 e rt BVSP, respectivamente) e variações na dependência (parâmetro $\rho_{t}$ da cópula t de Student) estimada para os dados SP500 X BVSP. 
Alguns trabalhos sugerem que diferentes regimes de dependência podem ser encontrados em dados do mercado financeiro (Rodriguez, 2007; Patton, 2006b; Filho, 2010). Deste modo, uma opção interessante para trabalhos futuros seria o estudo de modelos com mudança de regime.

Dentre outros assuntos a serem explorados, podemos destacar, a obtenção de novos modelos de cópulas a partir de misturas, comparações entre as equações de evolução já existentes para estruturas de dependência, estudos de simulação (similares aos realizados no capítulo 4) para verificar a taxa de seleção de modelos obtidos pelas cópulas variantes no tempo, com e sem mudança de regime, e finalmente aplicações relacionadas a contágio de mercado e VaR (value at risk). 


\section{Referências Bibliográficas}

Abbara, O. M. F. (2009). Modelagem de Depêndencia em Séries Financeiras Multivariadas. Dissertação de mestrado, Departamento de Estatística do Instituto de Matemática, Estatística e Computacao Científica da Universidade Estadual de Campinas, Campinas-SP.

Akaike, H. (1973). Information theory and an extension of the maximum likelihood principle, volume 1, pages 267-281. Akademiai Kiado.

Arakelian, V. \& Dellaportas, P. (2012). Contagion determination via copula and volatility threshold models. Quantitative Finance, 12(2), 295-310.

Ausin, M. C. \& Lopes, H. F. (2010). Time-varying joint distribution through copulas. Computational Statistics \& Data Analysis, 54(11), 2383-2399.

Azzalini, A. (1985). A class of distributions which includes the normal ones. Scandinavian Journal of Statistics, 12, 171-178.

Azzalini, A. (2011). R package sn: The skew-normal and skew-t distributions (version 0.4-17). Università di Padova, Italia.

Bollerslev, T. (1986). Generalized autoregressive conditional heteroskedasticity. Journal of Econometrics, 31(3), 307 - 327.

Chib, S. \& Greenberg, E. (1995). Understanding the Metropolis-Hastings Algorithm. The American Statistician, 49(4), 327-335. 
Clayton, D. G. (1978). A model for association in bivariate life tables and its application in epidemiological studies of familial tendency in chronic disease incidence. Biometrika, 65(1), 141-151.

Demarta, S. \& Mcneil, A. J. (2004). The t copula and related copulas. Technology, 73(1), 1-20.

Ehlers, R. S. (2012). Computational tools for comparing asymmetric GARCH models via Bayes factors. Mathematics and Computers in Simulation, 82, 858-867.

Embrechts, P., A. M. \& Straumann, D. (2002). Correlation and dependence in risk management: properties and pitfalls, Risk Management: Value at Risk and Beyond. Cambridge University Press, Cambridge.

Embrechts, P., L. F. \& McNeil (2003). Modelling Dependence with Copulas and Applications to Risk Management,em Rachev S.T. (ed.), Handbook of Heavy Tailed Distributions in Finance. Elsevier/North-Holland, Amsterdam.

Engle, R. F. (1982). Autoregressive conditional heteroscedasticity with estimates of the variance of United Kingdom inflation. Econometrica, 50(4), pp. 987-1007.

Fermanian, J.-D. \& Scaillet, O. (2004). Some statistical pitfalls in copula modeling for financial applications. FAME Research Paper Series rp108, International Center for Financial Asset Management and Engineering.

Fernandez, C. \& Steel, M. F. J. (1998). On bayesian modeling of fat tails and skewness. Journal of the American Statistical Association, 93(441), pp. 359-371.

Filho, O. (2010). Cópulas Tempo-Variantes em Finanças. Ph.D. thesis, Universidade Federal do Rio Grande do Sul, Programa de Pós-Graduação em Economia, Porto Alegre, Brasil. 
Forbes, K., R. R. (2002). No contagion, only interdependence: measuring stock markets comovements. The Journal of Finance, 57, 2223-2261.

Galassi, M., Davies, J., Theiler, J., Gough, B., Jungman, G., Alken, P., Booth, M. \& Rossi, S. (2010). GNU Scientific Library Reference Manual (3rd Ed.). ISBN 0954612078.

Green, P. J. (1995). Reversible jump Markov chain Monte Carlo computation and bayesian model determination. Biometrika, 82(4), pp. 711-732.

Hansen, B. E. (1994). Autoregressive conditional density estimation. International Economic Review, 35(3), 705-730.

Hastings, W. K. (1970). Monte Carlo sampling methods using Markov chains and their applications. Biometrika, 57(1), 97-109.

Joe, H. (1997). Multivariate Models and Dependence Concepts. Monographs on Statistics and Applied Probability. Chapman \& Hall.

Joe, H. \& Xu, J. J. (2008). The estimation method of inference functions for margins for multivariate models. Technical report, Department of Statistics, University of British Columbia.

Jondeau, E. \& Rockinger, M. (2006). The Copula-GARCH model of conditional dependencies: An international stock market application. Journal of International Money and Finance, 25(5), 827-853.

Kojadinovic, I. \& Yan, J. (2010). Modeling multivariate distributions with continuous margins using the copula R package. Journal of Statistical Software, 34(9), 1-20.

Liu, Y. \& Luger, R. (2009). Efficient estimation of copula-GARCH models. Computational Statistics $\&$ Data Analysis, 53(6), 2284-2297. 
Metropolis, N., Rosenbluth, A. W., Rosenbluth, M. N., Teller, A. H. \& Teller, E. (1953). Equation of state calculations by fast computing machines. The Journal of Chemical Physics, 21(6), 1087-1092.

Morettin, P. A. \& Toloi, C. (2004). Análise de Séries Temporais. Edgard Blucher, São Paulo.

Nelsen, R. B. (2006). An Introduction to Copulas. Springer, New York.

Nelson, D. B. (1991). Conditional heteroskedasticity in asset returns: A new approach. Econometrica, 59(2), pp. 347-370.

Patton, A. (2006a). Modelling asymmetric exchange rate dependence. International Economic Review, 47(2), 527-556.

Patton, A. (2006b). Estimation of multivariate models for time series of possibly diferent lengths. Journal of Applied Econometrics, 21(2), 147-173.

Patton, A. (2008). Copula-based models for financial time series. Ofrc working papers series, Oxford Financial Research Centre.

Plummer, M., Best, N., Cowles, K. \& Vines, K. (2006). CODA: Convergence Diagnosis and Output Analysis for MCMC. R News, 6(1), 7-11.

R Development Core Team (2010). R: A Language and Environment for Statistical Computing. R Foundation for Statistical Computing, Vienna, Austria. ISBN 3900051-07-0.

Roch, O. \& Alegre, A. (2006). Testing the bivariate distribution of daily equity returns using copulas. an application to the spanish stock market. Computational Statistics \&amp; Data Analysis, 51(2), 1312 - 1329.

Rodriguez, J. C. (2007). Measuring financial contagion: A copula approach. Journal of Empirical Finance, 14(3), 401-423. 
Schwarz, G. (1978). Estimating the dimension of a model. The Annals of Statistics, 6(2), pp. 461-464.

Silva, R. D. \& Lopes, H. F. (2008). Copula, marginal distributions and model selection: a bayesian note. Statistics and Computing, 18(3), 313-320.

Spiegelhalter, D. J., Best, N. G., Carlin, B. P. \& van der Linde, A. (2002). Bayesian measures of model complexity and fit (with discussion). Journal of the Royal Statistical Society, Series B, 64, 583-639(57).

Tierney, L. (1994). Markov chains for exploring posterior distributions. Annals of Statistics, 22, 1701-1762.

Werner \& Hürlimann (2004). Fitting bivariate cumulative returns with copulas. Computational Statistics Eamp; Data Analysis, 45(2), 355 - 372.

Yan, J. (2007). Enjoy the joy of copulas: With a package copula. Journal of Statistical Software, 21(4), 1-21.

Zhang, J. \& Guegan, D. (2008). Pricing bivariate option under GARCH processes with time-varying copula. Documents de travail du Centre d'Economie de la Sorbonne b08015, Universitè Panthèon-Sorbonne (Paris 1), Centre d'Economie de la Sorbonne. 


\section{Apêndice:}

\section{Gráficos das cadeias de Markov}

Abaixo são apresentados os gráficos obtidos pelas cadeias de Markov dos algoritmos MCMC dos modelos com menor DIC. Os demais gráficos foram omitidos por concisão.

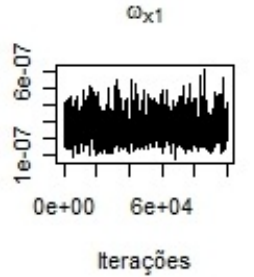

$7 x 1$

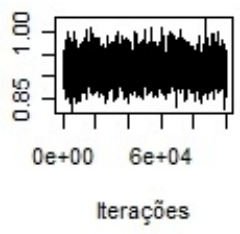

$v_{x 2}$

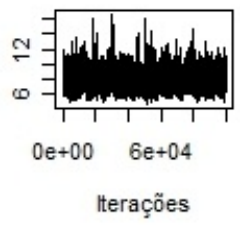

$\beta_{\mathrm{C}}$

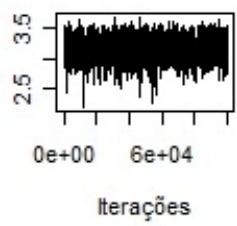

$\alpha_{\mathrm{x} 1}$

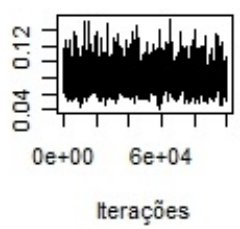

$\omega_{x 2}$

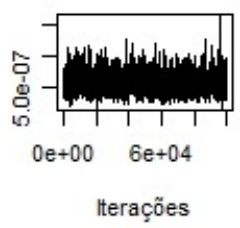

$7 \times 2$

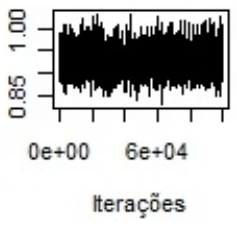

$\omega_{A R x 1}$

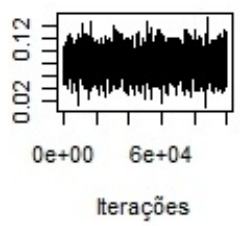

$\beta_{\mathrm{x} 1}$

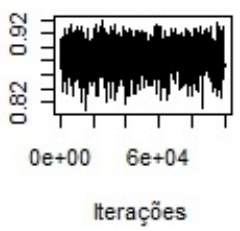

$\alpha_{x 2}$

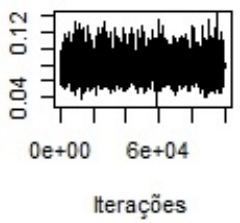

$\omega_{\mathrm{C}}$

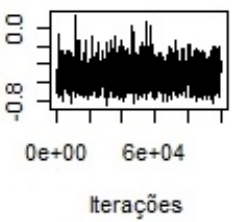

$v_{\mathrm{x} 1}$

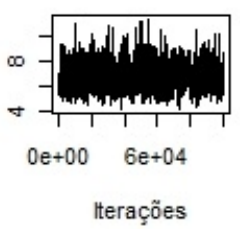

$\beta_{x 2}$

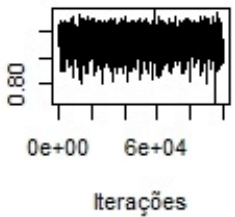

$\alpha_{C}$

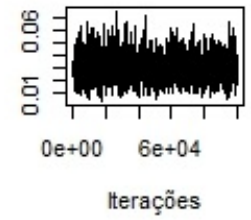

Figura 10: Cadeia de Markov dos parâmetros obtida para o ajuste do modelo com menor DIC (neste caso, o obtido a partir da cópula t de Student variante no tempo) ao par de índices ICP X BVSP. 
$\omega_{\mathrm{x} 1}$

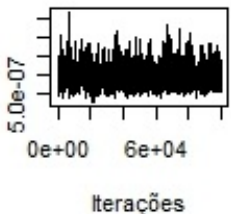

$7 \times 1$

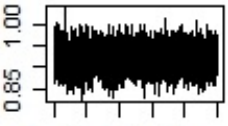

$0 \mathrm{e}+00 \quad 6 \mathrm{e}+04$

Iterações

$v_{x 2}$

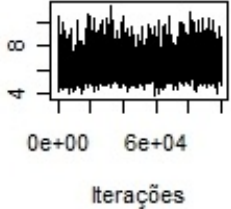

$\beta_{\mathrm{C}}$

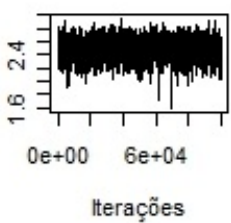

$\alpha_{\mathrm{x} 1}$

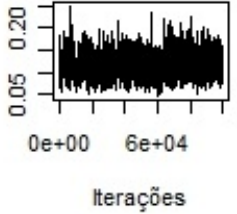

$\omega_{x 2}$

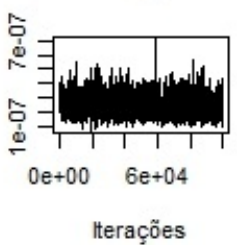

$7 \times 2$

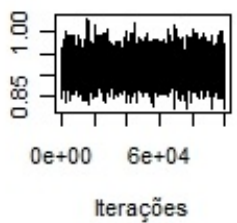

$\omega_{\mathrm{ARx} 1}$

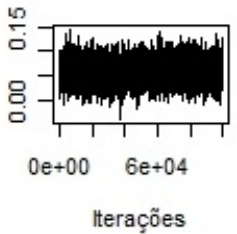

$\beta_{x 1}$

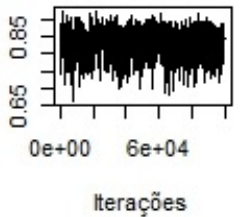

$\alpha_{x 2}$

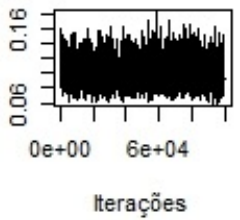

$\omega_{\mathrm{C}}$

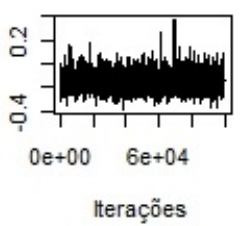

$\omega_{A R \times 2}$

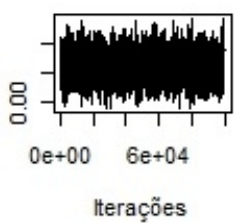

$\mathrm{v}_{\mathrm{x} 1}$

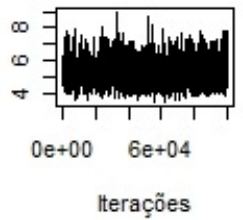

$\beta_{x 2}$

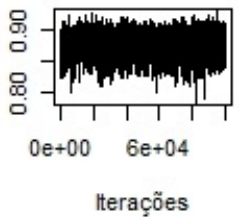

$\alpha c$

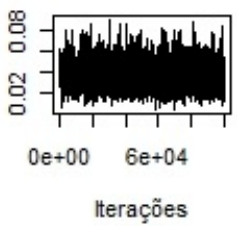

Figura 11: Cadeia de Markov dos parâmetros obtida para o ajuste do modelo com menor DIC (neste caso, o obtido a partir da cópula Normal variante no tempo) ao par de índices MERV X ICP. 
$\omega_{\mathrm{x} 1}$

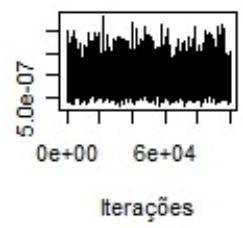

$7 x 1$

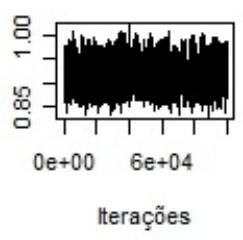

$v_{x 2}$

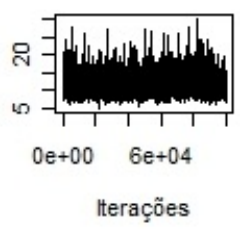

$\beta_{\mathrm{C}}$

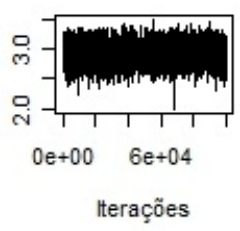

$\alpha_{x 1}$

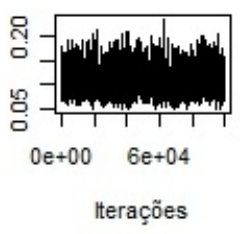

$\omega_{x 2}$

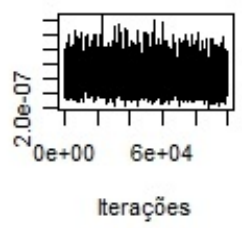

$7 \times 2$

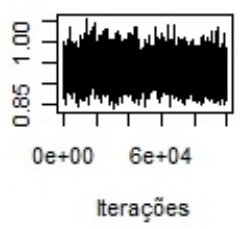

$\omega_{A R x 1}$

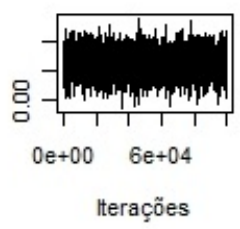

$\beta_{\mathrm{x} 1}$

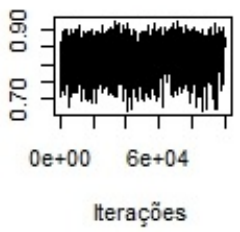

$\alpha_{x 2}$

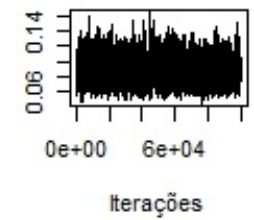

$\omega_{\mathrm{C}}$

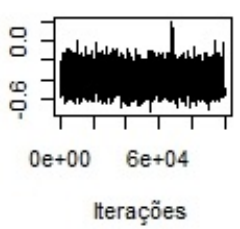

$v_{x 1}$

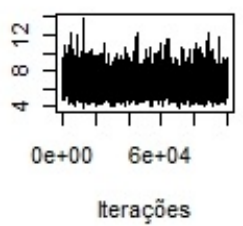

$\beta_{x 2}$

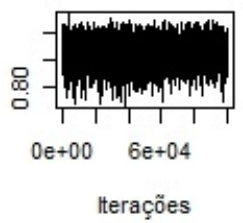

$\alpha_{c}$

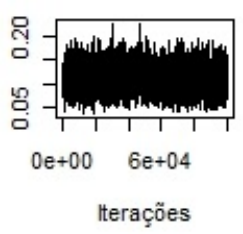

Figura 12: Cadeia de Markov dos parâmetros obtida para o ajuste do modelo com menor DIC (neste caso, o obtido a partir da cópula t de Student variante no tempo) ao par de índices MERV X BVSP. 
$\omega_{\mathrm{x} 1}$

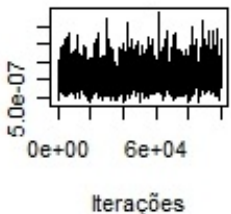

$7 \mathrm{x} 1$

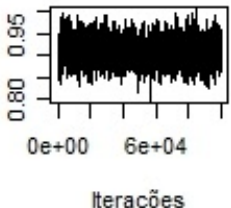

$v_{x 2}$

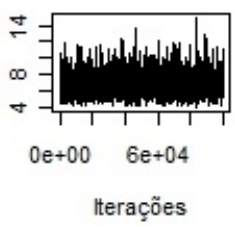

$\beta_{\mathrm{C}}$

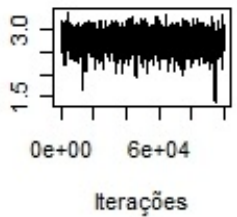

$\alpha_{x 1}$

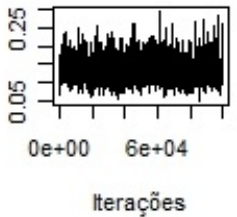

$\omega_{x 2}$

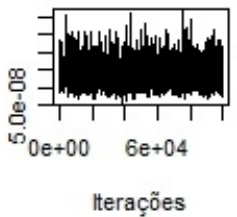

$7 \times 2$

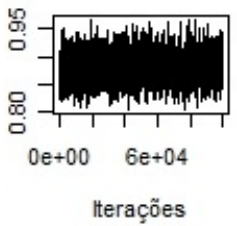

$\omega_{A R x 1}$

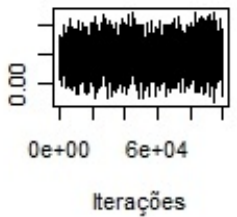

$\beta_{x 1}$

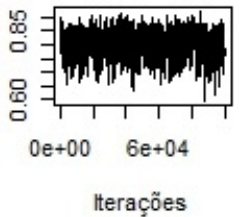

$\alpha_{\times 2}$

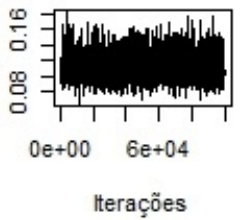

$\omega_{\mathrm{C}}$

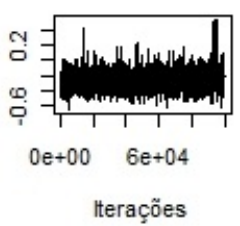

$\omega_{A R \times 2}$

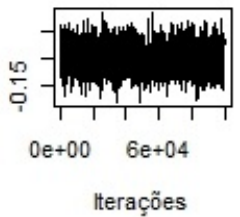

$\mathrm{v}_{\mathrm{x} 1}$

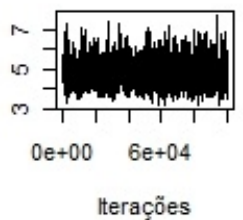

$\beta_{x 2}$

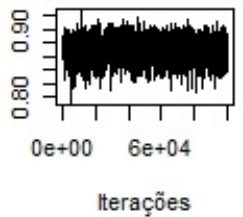

$\alpha_{C}$

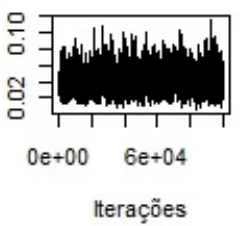

Figura 13: Cadeia de Markov dos parâmetros obtida para o ajuste do modelo com menor DIC (neste caso, o obtido a partir da cópula t de Student variante no tempo) ao par de índices MERV X SP500. 
$\omega_{\mathrm{x} 1}$

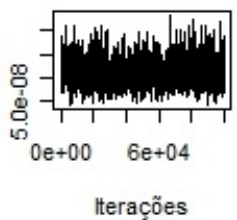

$7 \times 1$

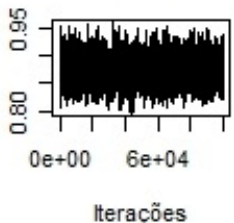

$v_{x 2}$

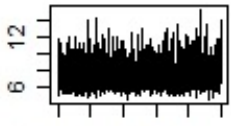

$0 \mathrm{e}+00 \quad 6 \mathrm{e}+04$

Iterações

$\beta_{\mathrm{C}}$

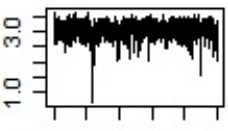

$0 \mathrm{e}+00 \quad 6 \mathrm{e}+04$

Iterações $\alpha_{\mathrm{x} 1}$

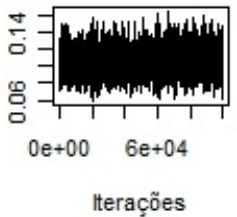

$\omega_{x 2}$

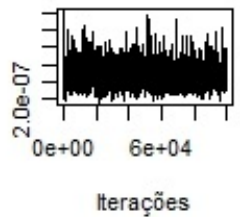

$7 \times 2$

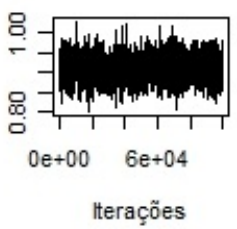

$\omega_{A R \times 1}$

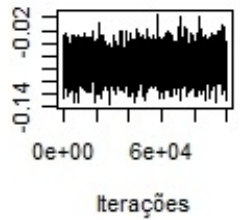

$\beta_{\mathrm{x} 1}$

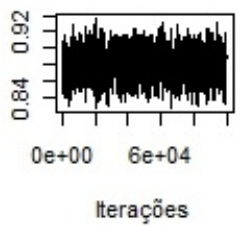

$\alpha_{x 2}$

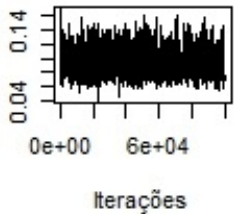

$\omega_{\mathrm{C}}$

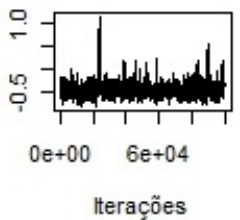

$v_{x 1}$

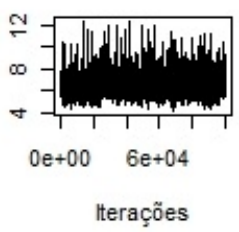

$\beta_{x 2}$

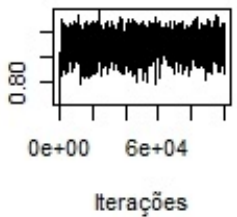

$\alpha_{c}$

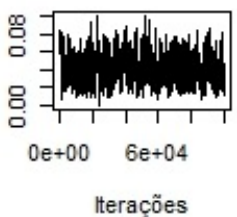

Figura 14: Cadeia de Markov dos parâmetros obtida para o ajuste do modelo com menor DIC (neste caso, o obtido a partir da cópula t de Student estática) ao par de índices SP500 X BVSP. 


$$
\omega_{\mathrm{x} 1}
$$

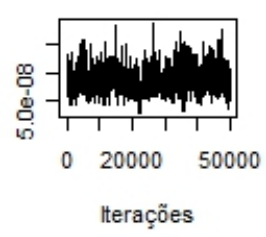

$7 x 1$

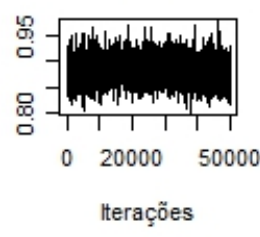

$v_{x 2}$

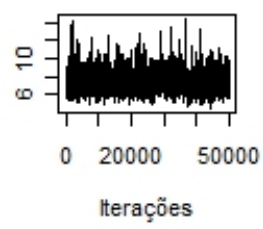

$\omega_{A R \times 2}$

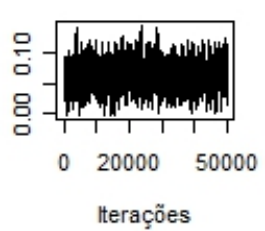

$\alpha_{\mathrm{x} 1}$

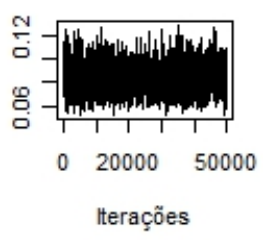

$\omega_{\times 2}$

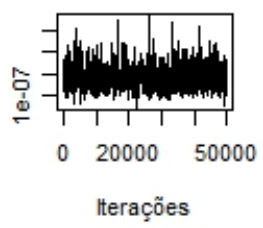

$7 \times 2$

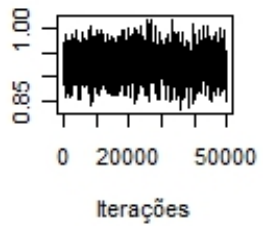

$\beta_{\mathrm{x} 1}$

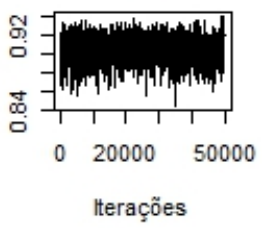

$\alpha_{x 2}$

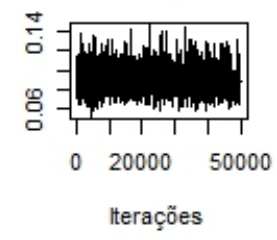

PC

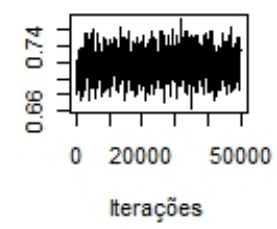

$v_{x 1}$

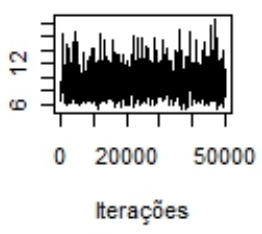

$\beta_{x 2}$

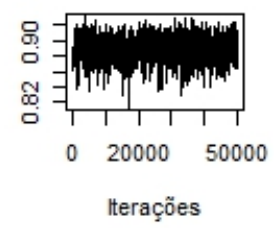

$\omega_{A R \times 1}$

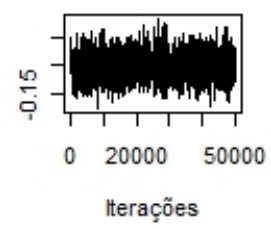

Figura 15: Cadeia de Markov dos parâmetros obtida para o ajuste do modelo com menor DIC (neste caso, o obtido a partir da cópula t de Student variante no tempo) ao par de índices SP500 X IPC. 